\title{
Understanding adolescents' experiences of self-harm: secondary analysis of Family Therapy sessions from the SHIFT trial
}

\author{
Robert Adam Holliday \\ Submitted in accordance with the requirements for the degree of \\ Doctor of Clinical Psychology (D. Clin. Psychol.) \\ The University of Leeds \\ School of Medicine \\ Academic Unit of Psychiatry and Behavioural Sciences
}

June, 2017 
The candidate confirms that the work submitted is his own and that appropriate credit has been given where reference has been made to the work of others.

This copy has been supplied on the understanding that it is copyright material and that no quotation from the thesis may be published without proper acknowledgement.

The right of Robert Holliday to be identified as Author of this work has been asserted by him in accordance with the Copyright, Designs and Patents Act 1988.

(C) 2017 The University of Leeds and Robert Holliday 


\section{ACKNOWLEDGEMENTS}

I am grateful to the participants of the original SHIFT trial who agreed for their sessions to be recorded. It was a privilege to hear their stories. I would like to thank my supervisors Dr Cathy Brennan and Professor David Cottrell for their guidance and support. Finally, I would like to thank my wonderful fiancé for her love, encouragement and help through this journey. To my parents and family for their love, support and belief in me throughout my life. I'm eternally grateful. To my friends, for keeping me grounded. 


\begin{abstract}
Research suggests that self-harming behaviour has increased and rates of self-harm are consistently higher in adolescence compared to adulthood. Understanding why adolescents engage in self-harming behaviour is important. Adolescents who selfharm are at higher risk of a repeated episode and self-harm is a key risk factor in completed suicide. Only a small number of studies have directly explored adolescents' views of their self-harm using first-hand accounts. Data was gathered via a secondary analysis of video-recorded Family Therapy sessions collected as part of the Self-harm intervention: Family Therapy. Session recordings of 22 participants, approximately 170 hours of footage, formed the dataset. Data was only transcribed data for later analysis if the adolescent was directly involved in the conversation. Using thematic analysis to analyse the data, five core themes were developed; (1) Distress can be difficult to convey (2) Self-harm and suicidal ideation; a complex relationship (3) Selfharm as a form of communication (4) Self-harm to manage emotions and (5) Moving forward. Prominent social discourses around self-harm resulted in some adolescents attempting to manage alone and fearing the consequences if they talked about selfharm. Accounts highlighted the complex interplay between self-harm and suicidal intent; for some this fluctuated across episodes of self-harm. Self-harm was a means of communicating distress as well as managing emotions. Encouragingly, many participants described being able to resist self-harm, often mirroring why some adolescents harmed themselves in the first place. Findings from the analysis are discussed in relation to the literature along with strengths, limitations, clinical implications and future research.
\end{abstract}




\section{ABBREVIATIONS}

DA: Discourse Analysis

CAMHS: Child and Adolescent mental health services

FASM: Functional Assessment of Self-Mutilation scale

FM: Family member

FT: Family Therapy

IPA: Interpretive Phenomenological Analysis

LAC: Looked after children

LACS: Leeds Attributional Coding System

LFTRC: Leeds Family Therapy and Research Centre Systemic Family Therapy

NCRM: National Centre for Research Methods

NICE: National Institute for Health and Care Excellence

NSSI: Non-Suicidal Self-injury

P: Participant

SHIFT: Self-harm intervention: Family Therapy

T: Therapist

TA: Thematic Analysis

TAU: Treatment as usual 


\section{TABLE OF CONTENTS}

ACKNOWLEDGEMENTS...................................................................................... 3

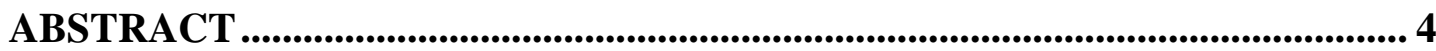

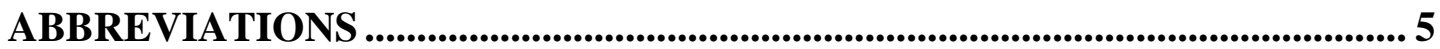

LIST OF TABLES \& FIGURES ........................................................................... 9

CHAPTER ONE: INTRODUCTION ................................................................ 10

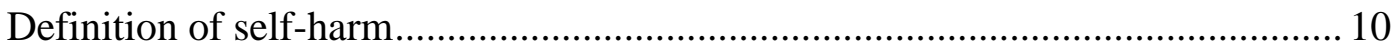

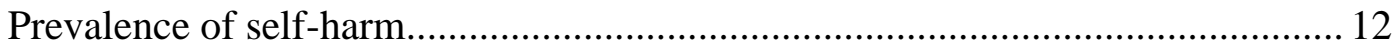

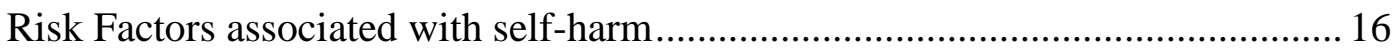

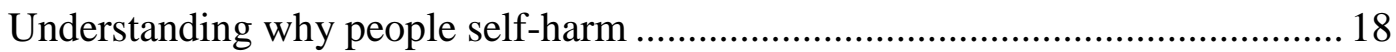

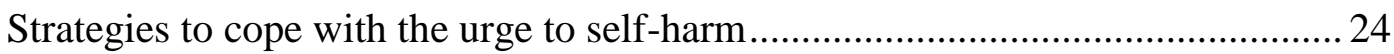

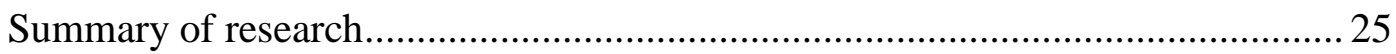

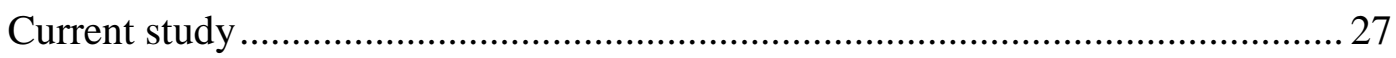

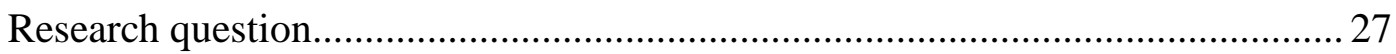

CHAPTER TWO: METHOD......................................................................... 28

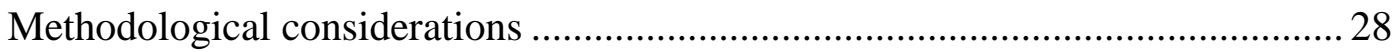

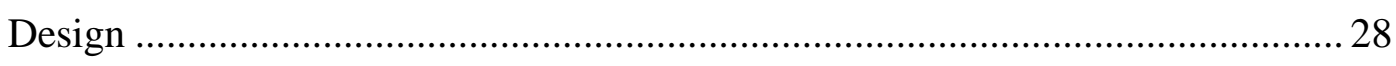

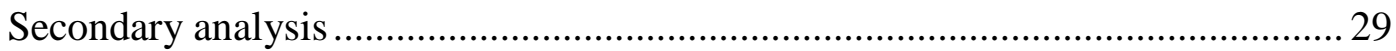

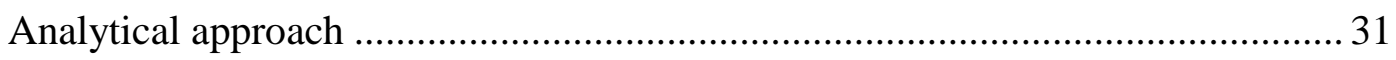

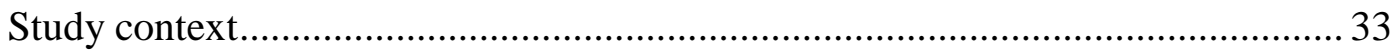

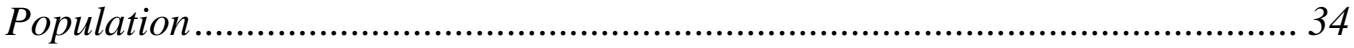

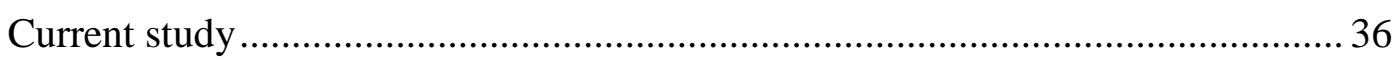

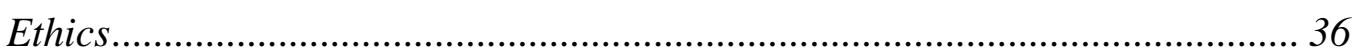

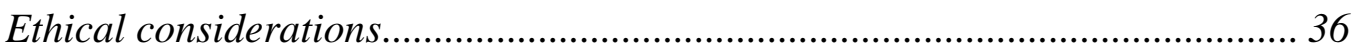

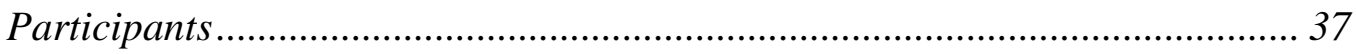

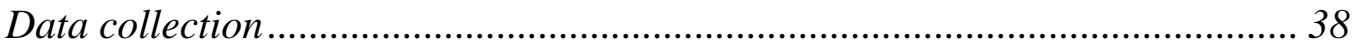

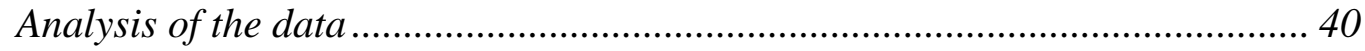

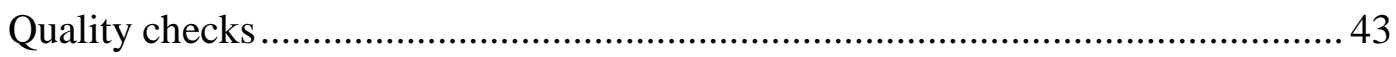

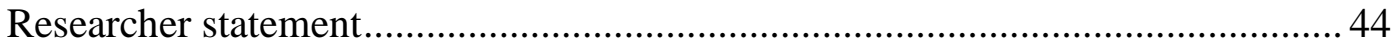

CHAPTER THREE: RESULTS......................................................................... 45

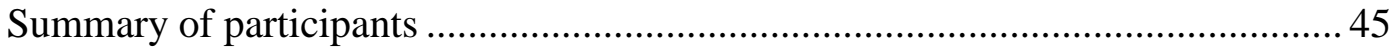


Setting the scene.....

Core themes. 48

Theme 1: Distress can be difficult to convey .......................................................50

Sub-theme 1: Trying to manage alone ................................................................ 50

Sub-theme 2: Feared consequences ............................................................... 50

Theme 2: Self-harm \& suicidal ideation: a complex relationship..........................52

Sub-theme 1: Clear articulation of intent to die ................................................ 52

Sub-theme 2: Self-harm \& suicidal ideation co-existing ................................. 55

Sub-theme 3: Self-harm but not wanting to die................................................ 56

Theme 3: Self-harm as a form of communication ............................................ 57

Sub-theme 1: Not a cry for help .................................................................... 57

Sub-theme 2: So others would listen ................................................................ 58

Sub-theme 3: Expressing distress without the consequences............................ 59

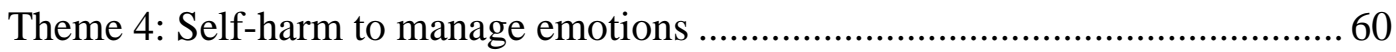

Sub-theme 1: Considered vs. uncontrolled self-harm ....................................... 60

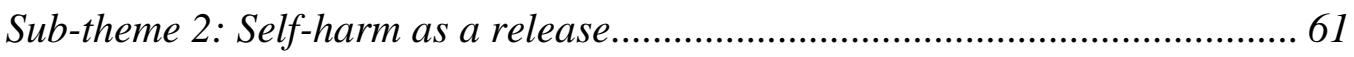

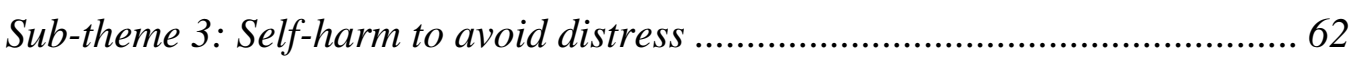

Sub-theme 4: Self-harm as a form of stimulation ........................................... 63

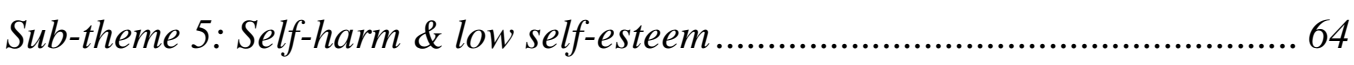

Sub-theme 6: Self-harm \& self-blame ............................................................... 65

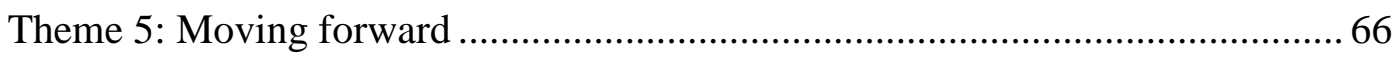

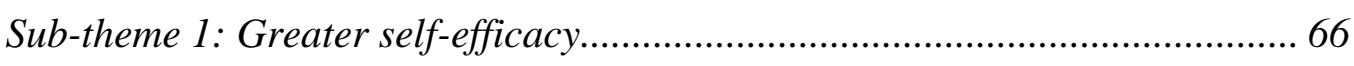

Sub-theme 2: Improved emotional regulation....................................................6 67

Sub-theme 3: Being heard, validated \& understood ........................................ 68

Sub-theme 4: Taking a step back ..................................................................... 70

Sub-theme 5: Distraction \& substitute behaviours ........................................... 73

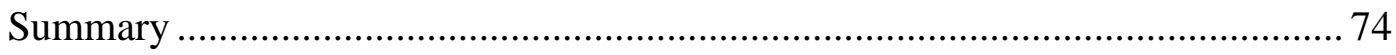

CHAPTER FOUR: DISCUSSION ................................................................... 76

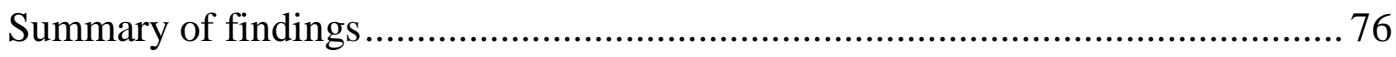

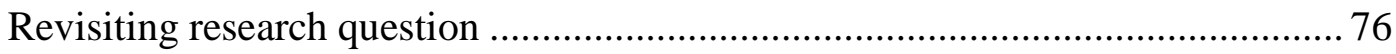

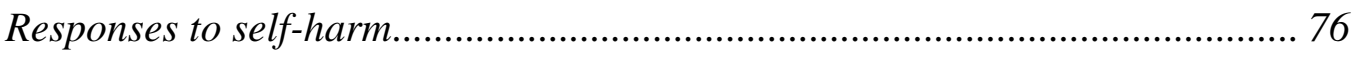

Understanding why adolescents' self-harmed ................................................ 80

How adolescents resisted, or ceased, self-harming ....................................... 84

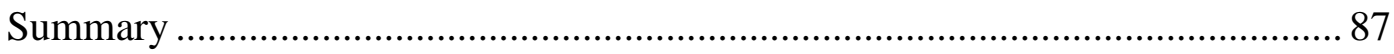




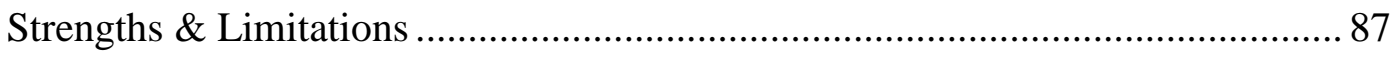

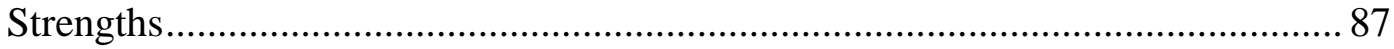

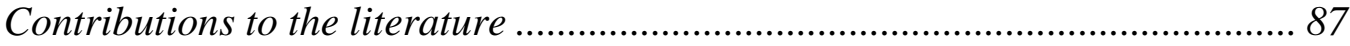

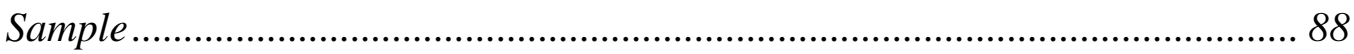

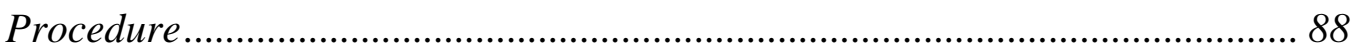

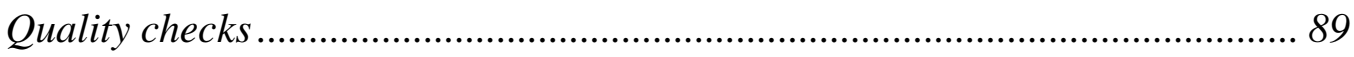

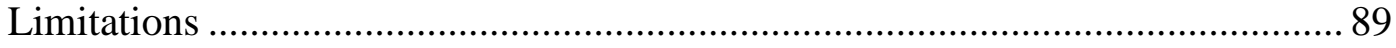

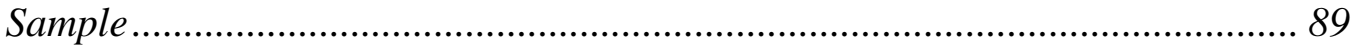

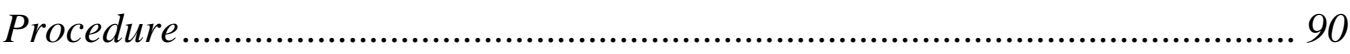

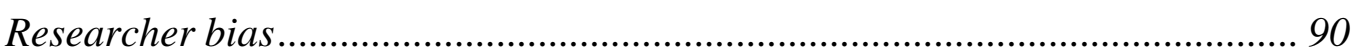

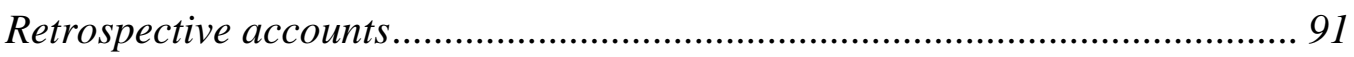

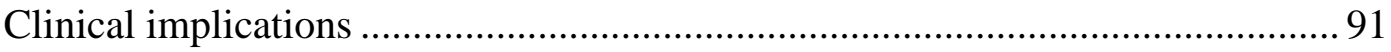

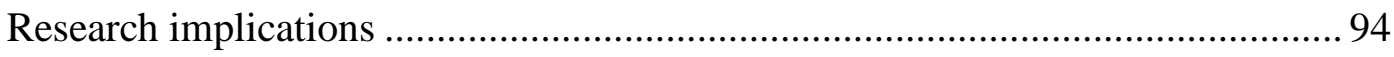

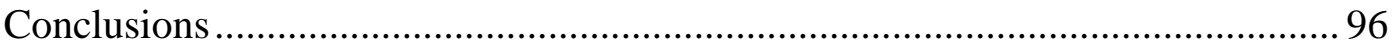

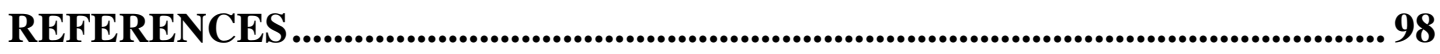

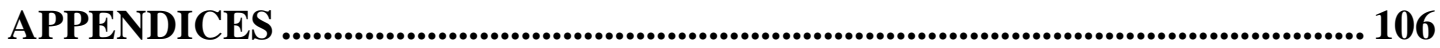

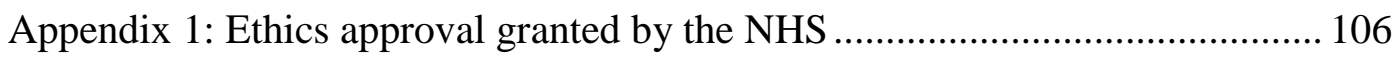

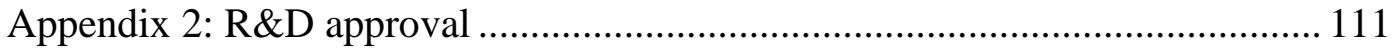

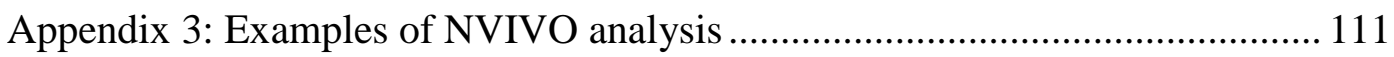




\section{LIST OF TABLES \& FIGURES}

Table 1: A checklist for achieving good TA (Braun \& Clarke, 2006)...................... 40

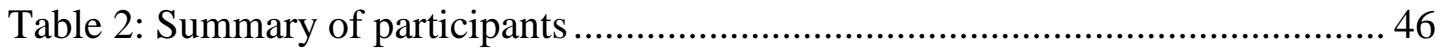

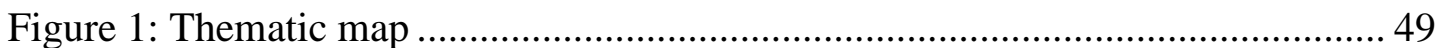




\section{CHAPTER ONE: INTRODUCTION}

Understanding why people, in particular adolescents, engage in self-harming behaviour is important for a number of reasons. Research suggests that self-harming behaviour has increased and is a public health concern (Rowe, French, Henderson, Ougrin, Slade \& Moran, 2014). There is a significant psychological and physical impact of this behaviour on the person, and it is often very distressing for friends and family (Nock, 2010). Self-harm is known to be positively correlated with a variety of adolescent risk taking behaviours, for example binge drinking and drug taking, as well as co-morbid mental health difficulties, such as low mood and anxiety (Brunner et al, 2014). Most importantly, adolescents who self-harm are at higher risk of a repeated episode and self-harm is a key risk factor in completed suicide (Klonsky, May \& Glenn, 2013).

Investigating personal accounts of self-harm can educate health professionals about the sensitivities and complexities they will encounter when gathering information during clinical assessments and therapies (Klineberg, Kelly, Stansfield \& Bhui, 2013). Understanding an adolescent's perspective of their self-harm is important as this information would inform the formulation, which underpins treatment. For example, if the function of self-harm is affect regulation then treatment would be focussed on helping the adolescent to manage emotive states. On a wider level, exploring personal accounts may contribute to further understanding of self-harm in adolescents.

\section{Definition of self-harm}

There are a number of, often conflicting, definitions of self-harm used in the literature. I will use the definition outlined in the National Institute for Health and Care Excellence (NICE) guideline CG133 'Self-harm in over 8s: long-term management' (NICE, 2011), referred to as "any act of self-poisoning or self-injury carried out by an individual irrespective of motivation" (page 4). I will use this definition as it is broad - capturing poisoning - and does not distinguish self-harm based on intent. This definition does not include accidental harm, or harm caused by excessive alcohol use and/or recreational drug use. Neither does it include harm caused by the effect of starvation arising from anorexia nervosa (NICE, 2011).

Within the literature there is a distinction between behaviours in which bodily injury is the intended purpose of one's behaviour, such as cutting, burning and biting, and 
those in which it is an unintended by-product (Nock, 2010). Binge-drinking alcohol and smoking are behaviours typically performed to result in enjoyment, not with the intention of causing harm, and are considered in-directly harmful (Nock, 2010). Whilst this may not always be clear with individuals using substances, harming oneself may not necessarily be the primary objective. On the other hand, self-poisoning can be thought of as a directly harmful behaviour; it is difficult to imagine a person ingesting a substance that is likely to poison them for pleasure and without the intention of causing harm.

Whilst cutting is the most commonly identified method of self-harm reported in the literature (Madge et al 2008; Klonsky 2011; Bruner 2014) occurring in approximately 70-90\% of individuals who self-harm (Nock, 2010), there are a number of ways people harm themselves. For example, self-poisoning is often reported as the second most frequently identified method of self-harm (Hawton, Rodham, Evans \& Weatherall, 2002; Rodham, Hawton \& Evans, 2004; Madge, 2008; Moran et al, 2012) and other research has found that individuals have endorsed jumping from a height, strangulation (Hawton et al 2002) and biting (Zetterqvist, Lundh, Dahlstrom and Svedin, 2013).

While some people harm themselves using one method, for example cutting only, some individuals switch methods of self-harm between episodes. Owens et al (2015) found, in a study of individuals who had attended hospital for self-harm, that between episodes of self-harm a large number of people switched methods by which they had harmed themselves (22.5\% of 21,255 participants reported switching methods). Switching was more common among males (37\%) compared to females (33\%).

Several definitions of the phenomena described in previous research do not include self-poisoning as a form of self-harm, such as "self-mutilation" (Favazza, 1998), "deliberate self-injury" (Klonsky 2007) and "Non-suicidal self-injury" (Nock \& Favazza, 2009, cited in Zetterqvist, Lundh, Dahlstrom \& Svedin, 2013). I think it is important to incorporate self-poisoning in a definition of self-harm as it is the second most common method, behind cutting, by which people harm themselves and results in harm to the individual (Hawton, Rodham, Evans and Weatherall, 2002; Madge et al, 2008; Moran et al, 2012).

Both Nock (2010) and Muehlenkamp et al (2012) suggest the inconsistent use of terms is a significant barrier in accurately conceptualising self-harm and in conducting 
research in the area. If there is no universal definition of self-harm then it makes it very difficult to carry out research in the first instance; how can research be conducted on a phenomena if there is no clear agreement on how to define the phenomena. This could also be confusing for individuals, for example adolescents/parents/professionals, searching for information as they may be unaware of the subtle differences in terminology.

Some researchers suggest that definitions of self-harm should differentiate intent, such as suicidal vs. non-suicidal (Nock, 2010). However, there is on-going discussion as to whether a person's intent during self-harm can be reliably reported (Kapur, Cooper, O’Connor \& Hawton, 2013; Brunner et al 2014), as participants in research studies are often asked to do this retrospectively through self-report. It may have been sometime between the incident and the reporting of the act, or the person may have been unsure of their intention when they harmed themselves (Kapur et al, 2013). Some research excludes individuals who, retrospectively, reported being uncertain or expressed intent to complete suicide. Therefore, those individuals for whom intention was not clear or did not complete suicide are being omitted from some research despite having selfharmed. This potentially limits the applicability of the research as different views are not presented.

While there is an association between self-harm and suicide - a history of self-harm has been shown to increase the risk of completed suicide (Hawton, Rodham \& Evans, 2006) - an individual can self-harm but not go on to complete suicide. Victor and Klonsky (2014) found that historical suicidal ideation was the strongest predictor of attempted suicide whereas "self-injury" was only a moderate predictor.

\section{Prevalence of self-harm}

In order to explore if higher rates of self-harm are found among adolescents compared to adults - as proposed by Nock (2010) - it is necessary to examine the prevalence rates within the literature. One study of adults, using a community sample, suggested approximately $4 \%$ of adults had a history of what they termed "deliberate self-harm", defined as "the intentional injuring of one's own body without apparent suicidal intent" (page 1, Klonsky, Oltmanns \& Turkheimer, 2003). The sample was very particular; participants were 1,986 American military recruits with an average age of $20(\mathrm{SD}=5)$. 
The majority of participants were Caucasian. Mean IQ was 104 and 99\% were high school graduates.

Another study investigating the prevalence in adults in the community found a lifetime prevalence rate of 5.9\% (Klonsky, 2011). This study used Non-suicidal Self-injury (NSSI) as the definition of self-harm, which "refers to the intentional destruction of one's own body tissue without suicidal intent" (page 1). Age ranged from 19-92 with a mean of $55.5(\mathrm{SD}=16.6)$.

Swannell, Martin, Page, Hasking and St John (2014) reviewed studies of NSSI across ages (adolescence, young adulthood \& adulthood). For inclusion, studies were required to define their construct of interest as damage to the body which was "direct"; "intentional" and "non-suicidal" (page 1). Studies were not included if the sample comprised clinical - not specified - intellectually impaired, detained or incarcerated participants. In total, lifetime estimates of NSSI were derived from 231,553 respondents across six geographical regions (Asia, Australia/New Zealand, Canada, Europe, United Kingdom \& USA). A prevalence rate of $4.2 \%$ was found in adults (classified as aged over twenty-five). A higher rate of self-harm, 15.4\% was found amongst adolescents. This was higher compared to both young adults (10.5\%; aged 18-24) and adults (4.2\%; aged > 25). Rates were higher in females compared to males $(21.3 \%$ vs. $17.8 \%)$.

Moran et al (2012) investigated the course of self-harm, defined as "an act with a nonfatal outcome in which an individual deliberately initiates behaviour - such as selfcutting - or ingests a toxic substance or object, with the intention of causing harm to themselves" (page 1) from adolescence to young adulthood in a sample of Australian adolescents between 1992 and 1998. Participants entered the study at two time points; waves one (1992, mean age 14.9 years, SD 0.46) and two (1993, mean age 15.5 years, SD 0.49). Participants were subsequently reviewed at further four to six month intervals from 14-19 years (waves three to six) with three follow-up waves in young adulthood; aged 20-21 years (wave seven), 24-25 years (wave eight) and 28-29 years (wave nine). In waves one to six ("Adolescence") participants self-administered the questionnaire on laptop computers with telephone follow-up. Participants were not asked about self-harm until wave three when the researchers expected the participants to be more engaged. In waves seven to nine ("Young Adult") data was gathered using computer assisted telephone interviews. From a total sample of 2,032 students, 1,943 
participated at least once during waves one to six, with 1,395 following through to wave nine. Participants were asked if they had ever deliberately hurt themselves or done anything that they knew might have harmed or even killed them. Those who responded positively were then asked to provide detail. This was coded into five subtypes by a researcher and confirmed by a corresponding author. A yes/no variable was created for each subtype: cutting or burning, self-poisoning, deliberate nonrecreational risk-taking, self-battery and other, for example hanging, attempted selfdrowning and intentional electrocution. Self-harm was higher amongst adolescents (8.3\%; aged 15.9-17.4 years) compared to young adults (2.6\%; aged 20.7-29.1 years). Rates were higher among females in both the adolescent (10\% vs. 6.3\%) and young adult phase (3.2\% vs. $1.9 \%)$ compared to males.

Prevalence of self-harm is consistently higher in adolescence compared to adulthood (Nock, 2010; Moran et al, 2012; Swannell, Martin, Page, Hasking \& St John, 2014) although the estimates vary greatly between studies. Hawton, Rodham, Evans and Weatherall (2002) reported a lifetime prevalence rate of $13.2 \%$. Whereas, two more recent large scale studies researching self-harm in adolescence reported lifetime prevalence rates of 17.8\% (Madge et al, 2008) and 27.6\% (Brunner et al, 2014) respectively.

Hawton, Rodham, Evans and Weatherall (2002) investigated the lifetime prevalence of self-harm in adolescents in English schools. The termed used was deliberate selfharm defined as "an act with a non-fatal outcome in which the individual deliberately: initiated behaviour, for example cutting; ingested a substance in excess of the prescribed or generally recognised therapeutic dose; ingested a recreational or illicit drug that was an act the person regarded as self-harm; ingested a non-ingestible substance or object" (page 2). The sample included 6,020 students, although 1,243 students were absent on the day their questionnaire was administered, 23 students opted out and 139 students were opted out by parents, giving a response rate of $76 \%$. A self-harm rate of $13.2 \%$ was reported, with significantly more females $(11.2 \%)$ than males $(3.2 \%)$ having reported harming themselves.

Madge et al (2008) investigated the lifetime prevalence of deliberate self-harm in over 30,000 adolescents from seven European countries, aged 14-17, and found that $17.8 \%$ of adolescents reported having self-harmed. Females were more than three times as likely as males to report an episode of self-harm. Deliberate self-harm was defined as 
"an act with a non-fatal outcome in which the individual deliberately: initiated behaviour, for example cutting; ingested a substance in excess of the prescribed or generally recognised therapeutic dose; ingested a recreational or illicit drug that was an act the person regarded as self-harm; ingested a non-ingestible substance or object" (page 3).

Brunner et al (2014) carried out a large scale cross-sectional assessment of "direct selfinjurious behaviour" defined as "intentional self-inflicted damage to the surface of an individual's body, which includes self-cutting, burning, biting, hitting, and skin damage by other methods, regardless of the suicidal intent" (page 2) in adolescents in 11 European countries. They found a lifetime prevalence rate of $27.6 \%$, with significantly more females having reported self-harming. This definition did not include those individuals who had self-harmed by poisoning. Self-poisoning has been reported as the second most frequently identified method of self-harm behind cutting in the study by Hawton, Rodham and Evans (122 individuals; 30.7\% vs. 257 individuals 64.6\%, 2002) and Madge et al (6,796 individuals; 22.3\% vs. 17,036; $55.9 \%, 2008)$ meaning this definition did not capture a potentially large number of adolescents who were harming themselves using this method.

All three studies (Hawton et al 2002; Madge et al, 2008; Brunner et al, 2014) sampled a large number of adolescents using questionnaire measures administered in school. This excludes those adolescents who were absent on the day of the questionnaires being administered which has implications for the robustness of the results, as it may be the case that these prevalence rates do not represent the true scale of self-harm due to those students not present, such as those truanting or absent $(1,243$ in the study by Hawton et al 2002).

While the prevalence estimates described in the above studies differ, they highlight that self-harm is widespread among adolescents. It is likely that a number of factors contribute to this variance. For example, the range of definitions used to capture selfharming behaviour then impacts on the number of adolescents included in a sample as some are excluded. Other contributing factors include the age range used to capture the sample of adolescents, where the sample is drawn from, for example in-patient setting or community, and if the prevalence of self-harm is investigated over a certain period - such as the last 12 months - or over the lifetime, to name a few. 
It is also estimated that up to $50 \%$ of young people who self-harm do not seek help (Robinson, 2016). Even selecting the more conservative estimate, found in the study by Moran et al (2012), this suggests almost 1 in 10 adolescents may be engaging in self-harming behaviour. The upper estimates found in the studies by Madge et al (2008) and Brunner et al (2014) are far more alarming; suggesting nearly 1 in 5 to as many as 1 in 3 adolescents may be harming themselves.

\section{$\underline{\text { Risk Factors associated with self-harm }}$}

Self-harm in adolescents is the end product of a complex interplay between a number of factors, including genetic, biological, psychological, social and cultural (Hawton, Saunders \& O'Connor, 2012). A multitude of risk factors have been found to be associated with self-harming behaviour.

A systematic review by Fliege, Lee, Grimm and Klapp (2009) focused on the sociodemographic and psychological factors of "deliberate self-harm" defined as the “intentional self-inducing harming behaviour of one's own body resulting in relevant tissue damage" (page 1). Studies exploring only suicidal behaviour or self-poisoning were excluded, however the authors did include a small number of studies on suicidal behaviour provided they also looked at NSSI. A total of 59 papers met the inclusion criteria which stated that the content must include at least one correlate, risk factor or protective factor.

With regards to sociodemographic factors, higher rates of self-harm were found in adolescents and young adults compared to adults (Schmidtke et al 1996; Briere \& Gill, 1998 cited in Fliege et al, 2009) and more recent research has reported similar findings to the review (Moran et al, 2012; Swannell et al, 2014).

Life events and family adversity were risk factors explored in the review by Fliege et al (2009) and a number of studies demonstrated an association between traumatic experiences in childhood and self-harm. Most frequently found were associations between childhood experiences of emotional neglect (Akyuz, Sar, Kugu \& Dogan, 2005; Kirkcaldy, Brown, \& Siefen, 2006 cited in Fliege et al, 2009), psychological abuse (Gratz, Conrad \& Roemer, 2002; Akyuz et al, 2005 cited in Fliege et al, 2009), separation in childhood (Van Der Kolk, Perry \& Herman, 1991; Gratz, Conrad \& Roemer, 2002 cited in Fliege et al, 2009), physical abuse (Gratz, Conrad \& Roemer, 2002; Wright, Friedrich, Cinq-Mars, Cyr \& McDuff, 2004; Akyuz et al, 2005 cited in 
Fliege et al, 2009) and adolescent or adult self-harm. Twenty-one studies reported associations between self-harming behaviour and sexual abuse. Recent research has demonstrated similar findings to the review by Fliege et al (2009) highlighting the association between childhood mistreatment - all forms of childhood abuse; emotional/psychological, neglect/abuse including bullying, physical and sexual abuse - and self-harm (Hawton, Saunders \& O'Connor, 2012). Childhood adversity has also been shown to be a key risk factor for suicide in adolescence (Bjorkenstam, Kosidou \& Bjorkenstam, 2017).

Other family factors found to be strongly associated with self-harm include reduced parental involvement and parental unemployment (Brunner et al, 2014) and exposure to self-harm and suicide of others, for example family and friends, Hawton et al, 2012).

As well as antecedents (such as history of abuse), individual psychological factors, such as high levels of anxiety and low mood have been found to be associated with higher levels of self-harm in adolescents (Brunner et al, 2014). In several studies in the review by Fliege et al (2009), self-harm was associated with low self-esteem and low problem-solving abilities (Hawton, Rodham, Evans \& Weatherall, 2002; Portzky, De Wilde \& Van Heeringen, 2008 cited in Fliege et al, 2009). In both of these studies the following definition of deliberate self-harm was used "an act with a non-fatal outcome in which the individual deliberately: initiated behaviour (for example cutting); ingested a substance in excess of the prescribed or generally recognised therapeutic dose; ingested a recreational or illicit drug that was an act the person regarded as self-harm; ingested a non-ingestible substance or object". Individuals harming themselves also had higher tendency towards a self-blaming coping style and self-derogation than those individuals not harming themselves (Herpertz, Sass \& Favazza, 1997; De Leo \& Heller, 2004 cited in Fliege et al, 2009).

Self-harm also co-occurs with other harmful behaviours. For example the consumption of cigarettes, alcohol and illicit drugs have been found to be correlated with self-harm in adolescents (Hawton, Rodham, Evans \& Weatherall, 2002; Rasmussen, Miles \& Hawton, 2009), with females who engage in alcohol and substance abuse seemingly at higher risk of self-harm than males (Brunner et al, 2014). It is important to note the results from studies demonstrating correlation do not evidence causation. Similarly, those studies highlighting an association between a 
factor and self-harm do not demonstrate causation. For example, while self-harm has been shown to co-occur with other harmful behaviours, such as the consumption of alcohol and illicit drugs (Hawton et al, 2002), it may be that self-harm is a consequence of these behaviours as opposed to a causal factor, and vice versa.

\section{Understanding why people self-harm}

Within the literature there have been several theoretical attempts to understand why people self-harm. Suyemoto (1998) conducted a review and proposed six functional models of self-harm; environmental, drive (anti-suicide), sexual, affect-regulation, dissociation and interpersonal (boundaries), commenting that the affect-regulation and boundaries models had received the most empirical support in the literature. Suyemoto utilised "self-mutilation" as the term to describe the phenomena; "repetitive behaviour that causes minor to moderate physical injury" during which the individual "is not attempting suicide or responding to the need for self-stimulation" (page 2). This would appear to discount individuals who have self-harmed on a single occasion and it is not clear how we would distinguish minor from moderate self-harm. It may also be the case that the individual is unaware of intent to end their life when they harm themselves. Furthermore, it may be that 'the need for self-stimulation' is a motive for self-harming behaviour through a sense of sensation seeking (Edmondson, Brennan \& House, 2016). However, this review was a comprehensive examination of the literature and has been widely cited.

Klonsky (2007) reviewed the empirical research to identify functions of "self-injury", defined as the "intentional and direct injuring of one's body tissue without suicidal intent" (page 2). Methods were listed as skin-cutting, burning, scratching, banging/hitting body parts and interfering with wound healing. Initially, Klonsky proposed seven functions of self-harming behaviour; affect-regulation, antidissociation, anti-suicide, interpersonal boundaries, interpersonal-influence, selfpunishment and sensation-seeking. Of the 18 empirical studies analysed, 11 examined reasons for self-injury, with the average age in the studies ranging from 15-37 years old. Affect regulation was the most frequently selected reason for self-harm, both in adult and adolescent samples, with self-punishment the second most commonly cited function. The majority of samples were taken from in-patient or forensic populations. This excludes other individuals (such as those accessing community treatments) who may have given other reasons, not covered in the research, for why they had harmed 
themselves. Environmental factors may have influenced those participants in the inpatient/forensic populations which influenced why they self-harmed. Also, the definition used appears to exclude those for whom intent is unclear and who have poisoned as a means of self-harm.

Nock (2010) presented a review with an integrated theoretical model for the development and maintenance of self-injury - using the definition described in the above paragraph - and suggested the two main functions of self-injury are to regulate affective experience and regulate the social experience. The model makes three propositions; firstly self-injury is repeated because it functions as an immediately effective method of regulating one's affective/cognitive experience, and/or, influencing one's social environment in a desired way. Secondly, the risk of selfinjury is increased by factors that create a pre-disposition to have problems regulating one's affective/cognitive state or influencing one's social environment in general (for example hyper-arousal in response to stressful events \& poor verbal skills). The third proposition is that the risk of self-injury is increased by several self-injury specific factors that lead a person to choose this behaviour - over other maladaptive behaviours such as substance use.

Nock (2010) highlights the helpful distinction between behaviours in which bodily injury is the intended purpose of one's behaviour (such as directly self-injurious behaviours) and those in which it is an unintended by-product (for example in risky behaviours). However, he conceptualises self-harm, as Klonsky (2007) did, as "the direct and deliberate destruction of bodily tissue in the absence of suicidal intent" (page 4). These theoretical models outlined above focus only on the concept of 'selfinjury' and the definitions used would exclude those individuals who were not sure if they truly did want to end their life at the time of harming themselves, or could not recall their intentions.

Recently, Edmondson, Brennan and House (2016) conducted a systematic review of the literature reporting first-hand accounts for the reasons people self-harm other than intent to die. Self-harm was defined as "any intentional act of self-poisoning or selfinjury excluding only indirectly self-harming behaviour such as harmful alcohol or drug use, and regardless of the method of self-harm used" (page 2). To classify responses, a thematic analysis and 'best fit' framework analysis was undertaken. The initial framework included eight themes derived from reviews by Suyemoto (1998) 
and Klonsky (2007): affect-regulation, interpersonal influence, punishment, managing dissociation, sensation seeking, anti-suicide, expressing and coping with sexuality and maintaining or exploring boundaries. While in most studies affect regulation was the most commonly cited reason (for example "doing this relieved the emotional pain"), an additional three themes were proposed based on the data; "self-harm as a positive experience", "self-harm as a language" and "self-harm to achieve mastery". This study suggested that there may be positive reasons that help to explain why people repeatedly harm themselves. Studies eliciting first person accounts of functions of self-harm were included, as were questionnaire and survey based studies. While this study sheds further light on the reasons people harm themselves, and incorporates adaptive and potentially positive aspects of self-harm, the data was drawn from adults' accounts of the functions of self-harm, and did not include accounts from adolescents.

A number of primary research studies have been carried out to understand why adolescents self-harm. Nock and Prinstein (2004) examined reasons adolescents engage in "self-mutilative behaviour" in a sample of psychiatric inpatients. Ages ranged from 12-17. Similar to Suyemoto, the authors referred to self-mutilative behaviour as "deliberate damage to one's own body tissue without suicidal intent" (page 1). The Functional Assessment of Self-Mutilation Scale (FASM; Lloyd, Kelley \& Hope, 1997, cited in Nock \& Prinstein, 2004) was used to assess self-harming behaviours. The FASM is a self-reported measure of methods, frequency and functions of self-harm. The authors' proposed four theoretically driven functions; automatic reinforcement (positive), automatic reinforcement (negative), social reinforcement (positive) and social reinforcement (negative). The most frequently endorsed function was automatic reinforcement, which includes affect regulation. Whilst this study was one of the first to empirically demonstrate social reinforcement, it is not clear if the act of poisoning would be captured in "damage to one's own body tissue", thus potentially excluding individuals who had harmed themselves in this way. These people may have endorsed functions not captured in the results.

Lloyd-Richardson, Perrine, Dierker and Kelley (2007) investigated the functions of NSSI - defined as "deliberate, direct destruction or alteration of body tissue without conscious suicidal intent" (page 1) - in a community sample of 633 adolescents. Data was also gathered using the FASM. In this research self-harm was termed as "socially unacceptable, direct, repetitive and leads to minor or moderate harm". The authors 
suggested that the four-factor model put forward by Nock and Prinstein best fit their data based on analysis, with affect regulation being the most frequently cited function. The definition used in this research seemingly excludes those individuals who only self-harmed on one occasion and those who self-poison.

Both of the above studies employed the FASM to gather data (Nock and Prinstein (2004; Lloyd-Richardson et al, 2007). Participants completing the FASM are presented with 22 statements and asked to rate how often from never, to often, this motivation fits with their self-harm. Despite an 'other' box, the individual's response is constrained somewhat by the choices listed on the questionnaire. By ticking a response on the questionnaire, the reasons identified in research will reinforce those listed on that questionnaire and serve to reinforce the model the questionnaire is based on. It may be that this method of capturing data related to a person's motivation for self-harm doesn't fully capture that individual's experience of self-harm.

Hill and Dallos (2011) used narrative methodology to explore the way in which six adolescents ( 5 females, 1 male, aged between 13 and 18 years), who had been engaging in self-harm made sense of their self-harm and its relationship to events that had occurred in their lives. The term self-harm was used throughout the paper although no definition was provided. Participants self-selected from a population of adolescents accessing a charitable organisation working with young people who were experiencing, or at risk of developing, mental health difficulties. Participants were prompted to tell the story of their experiences using the life story interview. Common themes emerged across the six stories. Adolescents described a feeling of being misunderstood because those around them - parents, peers, professionals - did not understand their reasons for self-harm. They also described a sense of not being listened to. Hill and Dallos (2011) suggested this perception that others do not understand them, or their self-harm, seemed to be linked to the difficulty the adolescents experienced when discussing their self-harm. The authors commented how this appears to be a vicious circle; the adolescents found it difficult to talk to others about their self-harm as they found it difficult to understand it themselves. Another common theme described by the researchers was putting the anger inwards, which emerged through adolescents blaming themselves or believing they deserved to be punished. This study highlights the importance of allowing adolescents to gain a better understanding of themselves and their self-harming behaviour through reflective 
conversations. Hill and Dallos (2011) suggest that as these types of conversations are very difficult, adolescents and their families may require considerable assistance in structuring them. While the study explored the accounts of self-harm from the perspective of the adolescent, only six participants were sampled, with only one male participant. This means that it is very difficult to generalise these findings to other adolescents, who may provide differing accounts of self-harm and why they harmed themselves.

Klineberg, Kelly, Stansfield and Bhui (2013) conducted 30 individual interviews with an ethnically diverse sample of adolescents aged 15-16 (24 females, 6 males) in East London. The study aimed to increase understanding about how adolescents in the community talked about self-harm. NSSI was referred to as well but no definition was provided for either concept and they referred to the term self-harm throughout the research. They explored views on coping with stress, self-harm and attitudes towards, and experiences of, disclosure and help-seeking. Following a screening questionnaire administered to 319 participants in secondary schools, 30 participants were purposively selected.

Of the participants interviewed, ten had never self-harmed, nine had self-harmed on only one occasion, and eleven had self-harmed repeatedly. To ensure representation across different experiences and frequencies of self-harm, participants were selected on the basis of repetition (never, once, more than once) to include those adolescents who had self-harmed but not repeated the behaviour, and those who repeatedly hurt themselves. Adolescents who had not self-harmed were interviewed to explore peer attitudes. Participants were coded as having self-harmed if they had taken an overdose or harmed themselves in any other way at least once. Participants described a range of methods of self-harm including self-cutting, overdoses, self-battery, punching and self-burning. Most participants described numerous precipitants, including challenging relationships, family problems, exams, trouble with schoolwork and managing difficult feelings. Participants who had repeatedly self-harmed described a sense of relief, or knowing that harming themselves would make it better, with pain often being experienced following self-harm. In contrast, participants who had selfharmed once described a distance from their experience of self-harm. While participants were not directly asked about suicide, there were six in-direct references to suicidal ideation (Klineberg, Kelly, Stansfield \& Bhui, 2013). The adolescents who 
had not self-harmed expressed views that those who did self-harm were "attentionseeking" and "stupid". These adolescents also associated self-harm with suicide. Participants who had never self-harmed did say that they may not be able to fully comprehend the reasons for doing so. This study investigated self-harm from the firsthand perspectives of adolescents; however, the sample consisted of a majority of female participants (24/30). Although the researchers achieved some representation across different experiences with an ethnically diverse sample, it is not clear why such a high proportion of individuals purposively sampled were female. It does not state, for example, whether some male participants refused to be interviewed. It could have been that more male participants would have offered a perspective not captured in the data.

Palmer, Welsh and Tiffin (2016) explored perceptions of family functioning in adolescents who self-harm. They used the term self-harm defined as defined as "any act of self-poisoning or self-injury carried out by an individual irrespective of motivation" (page 2). Twenty-one adolescents presenting at four UK medical wards with self-harming behaviour were compared with two control groups. Control group data was created using pre-existing data collected in order to validate a tool to assess family perceptions. Control groups were matched on a 1:1 ratio with the self-harm group by gender, age and socioeconomic status. Participants in control group one were selected from three educational institutions. The control groups were designed to remove the presence of possible self-harming behaviour and other significant psychopathology. Therefore, participants scoring above the screening threshold for potential mental health problems were excluded. Participants in control group two were selected from all of the available community controls and matched on the same criteria as control group one, plus an emotional symptoms subscale score to create an emotionally matched control group.

Family perceptions were assessed using the family perceptions scale (FPS) which is a short, self-reported questionnaire validated for use in adolescents (Tiffin, 2007, cited in Palmer et al, 2016). Family perceptions were rated as significantly more dysfunctional on all subscales (except behavioural boundaries) in the self-harm group than in the control group. Similar differences were observed between the self-harm group and the emotionally matched control group, with significantly worse overall family perceptions in the self-harm group. Family perceptions data was available from 
fifteen of twenty one carers from the self-harm group, and highlighted poor levels of agreement between adolescents and carers on all measures of family perceptions.

\section{$\underline{\text { Strategies to cope with the urge to self-harm }}$}

Klonsky and Glenn (2008) utilised a structured interview to assess the prevalence and perceived helpfulness of methods for resisting self-injury, defined as "the deliberate and direct injuring of body tissue without suicidal intent for purposes not culturally sanctioned" (page 1) in a sample of young adults (mean age $=19.4, \mathrm{SD}=2.2$ ) with a history of skin-cutting and other "non-suicidal self-injurious behaviours". Participants were undergraduate students and a threshold of five or more cutting episodes was chosen to ensure a clinically relevant sample. Participants were presented with strategies that could be used to resist self-harm that were drawn from websites by, and for, those who self-harm.

'Keeping busy' and 'being around friends' were the most common methods identified for attempting to resist urges to self-harm (endorsed by $82.4 \%$ \& $80 \%$ respectively). Communicating was also heavily endorsed, either by talking to someone about their feelings (74.3\%), writing about feelings $(74.3 \%)$, talking to friends $(71.4 \%)$ or interacting with someone who was nice to them $(68.6 \%)$.

However, the most helpful methods identified to resist the urge to self-harm were 'doing sports or exercise recreationally' (65.2\% who used this method rated this as 'very' helpful) and 'removing the means used to self-harm' (63.6\% who used this method rated as 'very' helpful).

Interestingly, 'keeping busy' and 'being around friends' were only identified as being 'very' helpful by $35.7 \%$ and $32 \%$ of those who used these methods. Similarly, talking to someone about their feelings or talking to friends was only rated 'very' helpful by $38.5 \%$ and $32 \%$ of participants who had used this method.

Mumme, Mildred and Knight (2016) reviewed quantitative and qualitative literature exploring how non-suicidal self-injury cessation occurs, and individuals' experiences of stopping. NSSI was defined as "a self-directive and deliberate act that causes destruction or alteration of body tissue, without suicidal intent, and is seen as being socially unacceptable" (page 2). Two research questions were posed; what processes and strategies do people use to stop harming themselves and what are people's personal experiences of trying to stop, or having ceased, self-harm. 
Of the 454 papers identified through database searching, 9 were retained; 3 quantitative, 2 mixed-methods and 4 qualitative. Papers were excluded if they included treatment methods such as pharmacological and behavioural interventions and risk factors among others.

All three quantitative studies reported that those individuals who had self-harmed previously experienced significantly more family support than individuals who were currently self-harming. In two of the three quantitative studies, participants were aged over 18 suggesting that family support continues to be important beyond adolescence. Two of the qualitative studies also found family support to be an essential factor in self-harm cessation. Reasons included the helpfulness of having a strong role model and engaging in meaningful relationships.

In both the quantitative and qualitative papers, intrapersonal factors including selfesteem, self-efficacy, sense of hope and emotional regulation were found to influence self-harm cessation. Emotional regulation was found to be a significant factor for cessation, with individuals who had self-harmed previously demonstrating higher levels of emotional regulation including the ability to accept stronger emotions (Mumme, Mildred \& Knight, 2016).

Whilst there was an association between family support and self-harm this does not indicate causation; perhaps those who ceased self-harm then felt they were more supported by their family over time as opposed to this being the factor which triggered self-harm cessation.

\section{$\underline{\text { Summary of research }}$}

As highlighted, there are a number of definitions used to describe self-harming behaviour. This range of terminology proves to be a barrier in trying to make sense of the research to date as several of the definitions used capture slightly different phenomena. The variation is often highlighted between clinicians and researchers in the US and the UK and Western Europe, with practice in the US increasingly referring to deliberate self-harm and self-injury - usually associated with non-suicidal intent while in the UK and Western Europe self-harm usually encompasses self-injury and self-poisoning and the recognition that intent (suicidal vs. non-suicidal) is not always possible to demonstrate (Edmondson, Brennan \& House, 2016). 
Self-harm, in its broadest sense, is a common problem, particularly amongst adolescents. A range of methods have been identified by which adolescents harm themselves (Hawton, Rodham, Evans and Weatherall, 2002; Klonsky 2011; Zetterqvist, Lundh, Dahlstrom and Svedin, 2013; Bruner 2014) and a number of papers and studies have investigated the reasons why adults, and adolescents alike, harm themselves. Although the affect-regulation model has received a great deal of attention, and has been validated empirically (Nock \& Prinstein, 2004; LloydRichardson et al 2007) a number of studies have shown that self-harm occurs due to a number of interpersonal and intrapersonal reasons (Edmondson, Brennan \& House, 2016).

Several questionnaires have been used in research to explore self-harm, predominantly to assess prevalence and identify reasons given for self-harm, for example the Functional Assessment of Self-Mutilation scale (FASM, Lloyd, Kelley \& Hope, 1997, cited in Nock \& Prinstein, 2004) and the Self-harm behaviour questionnaire (Gutierrez, Osman, Barrios \& Kopper, 2001). One difficulty with the use of questionnaires to understand the reasons why people self-harm is that the very use of the measure limits the choices for the respondent. If the questionnaires are informed by reasons posited in previous literature then this only reinforces the reasons identified. Polkinghorne (2005) suggests that experience has depth, and short answer questionnaires gather only surface level information and are inadequate to capture the richness and fullness of an experience. Thus, the data gathered for the study of experience needs to consist of first-hand accounts (for example accounts provided verbally).

Laye-Gindhu and Schonert-Reichl (2005) suggest that it is important to canvas the views of adolescents directly, as the views of adults in isolation may not accurately reflect the actual experiences of adolescents. For example, family discord, parental criticism and peer relationship problems - arguably all more relevant in adolescence have been found to be closely linked to self-harm (Brunner et al, 2014) and may have a bearing on the reasons why adolescents self-harm.

The research by Palmer, Walsh and Tiffin (2016) demonstrates that disrupted family perceptions are associated with self-harm, with adolescents who are self-harming expressing significantly worse perceptions of their families compared to those not selfharming. Whilst this associated does not demonstrate causation, the review by 
Mumme, Mildred and Knight (2016) lends support to the idea that stronger family relationships are important in reducing self-harming behaviour, as this research found that family support was a key factor in self-harm cessation.

\section{Current study}

Whilst there has been a wealth of research focussing on the understanding of selfharm, particularly in the adult population, only a small number of studies have directly explored adolescents' views of their self-harm using first-hand, verbal, accounts (Hill \& Dallos, 2011; Brown \& Kimball, 2013; Klineberg, Kelly, Stansfield \& Bhui, 2013; Wadman et al, 2016). Brown and Kimball (2013) propose that a larger body of evidence is required that explores the unique experiences of those adolescents who self-harm to better understand the role and function of self-harm. The aim of my research is therefore to understand adolescents' experiences of self-harm.

\section{$\underline{\text { Research question }}$}

My research question was to understand adolescents' experiences of self-harm, specifically to explore their understandings of why they self-harmed, what their responses to self-harm were, and how they resisted or ceased self-harm. 


\section{CHAPTER TWO: METHOD}

\section{$\underline{\text { Methodological considerations }}$}

Whereas quantitative research uses numbers as data and analyses them using statistical techniques, qualitative approaches are generally engaged with exploring, describing and interpreting the personal and social experiences of participants (Smith, 2015) and use words as data (Braun \& Clarke, 2013). An attempt is usually made to understand a participant's frame of reference, or view of the world (Smith, 2015).

Qualitative research is seen as crucial for identifying and theorising different constructed versions of reality (Braun \& Clarke, 2013). Social constructionism proposes that as a culture and society we construct our own versions of reality between us (Burr, 2015) and the generation of knowledge and our concepts of 'reality' are generated through a social process (Haley, 2002). Social constructionism views language as one of the main ways in which we construct our social and psychological worlds (Burr, 2015).

Language is thought of as a fundamental property of human communication, interpretation and understanding (Burr, 2015) and one way in which we make sense of our social world (Smith, 2015). In qualitative research words are data, collected and analysed in various ways (Braun \& Clarke, 2013).

The use of qualitative methodology does have limitations. The data depends on the participant's ability to reflectively discern aspects of their own experience and to effectively communicate their reflections using language (Polkinghorne, 2005). One potential drawback of using languaged data is that people only have partial awareness, and not complete access, to their experiences. The translation of reflective awareness into languaged expression might not fully capture the experience, particularly if the person's view is offered some time after the experience actually occurred (Polkinghorne, 2005). Research quality is also heavily dependent on the individual skill set of the researcher and more easily influenced by the researcher's personal biases and idiosyncrasies (Anderson, 2010).

\section{$\underline{\text { Design }}$}

As the research was exploratory, qualitative methods were used to gain insight into how adolescents understood their experiences of self-harm. Data was gathered via a 
secondary analysis of video-recorded Family Therapy sessions collected as part of the SHIFT trial (Wright-Hughes et al, 2015).

\section{$\underline{\text { Secondary analysis }}$}

Secondary data analysis is a mode of inquiry with which to explore both qualitative and quantitative data. This involves the use of existing data, collected for the purposes of a prior study, in order to pursue a research interest which is distinct from that of the original work. Secondary analysis can involve the use of single or multiple data sets (Heaton, 2003).

This approach allows the researcher to maximise the usefulness of previously collected data and can be used to generate new knowledge, hypotheses or support for existing theories. It reduces the burden placed on participants by negating the need to recruit further and is a cost-effective approach to qualitative work (Heaton, 2003). Furthermore, existing data sets are often underused (Hinds, Vogel \& Clarke-Steffen 1997).

One limitation of secondary analysis as a method of approaching data is that the researcher is constrained by the existing data-set. For example, using a primary data collection method (such as semi-structured interview or focus group) would allow the opportunity to explore a participant's response in more detail. The researcher would be able to ask follow up questions to clarify a response and pursue a new line of enquiry.

I considered the use of primary data collection methods which would have allowed me to answer the research question in a more exploratory manner. However, I opted to use an existing data for two main reasons. Firstly, the original SHIFT study generated a wealth of data and participants had consented for their data to be used in future research projects. This data set allowed me to access more individuals than I would have been able to recruit and interview, and more data than I could collect (session recordings were collected for over 200 individuals). This offers breadth to the data to compensate for the inability to explore answers in more depth. Secondly, given that self-harm can be difficult to talk about, secondary analysis could be thought of as a more ethical approach compared to primary data collection methods, as there is no additional burden on the individual of having to discuss their experiences in more depth. 
Heaton (2003) suggests that there are three main forms of secondary data analysis;

1. Additional in-depth analysis: a more intensive focus on a particular finding or aspect than was undertaken for the primary research.

2. Additional sub-set analysis: a selective focus on a sub-set of the sample from the original study, sharing characteristics and warranting further analysis.

3. New perspective/conceptual focus: the retrospective analysis of the whole, or part, of the data set from a different perspective.

This research approached the data from a new perspective - taking part of the data-set and focussing on how adolescents understood their experiences of self-harm. The original research examined the effectiveness of family therapy as a treatment of selfharm compared to treatment as usual. If consent was obtained the family therapy sessions were recorded for use in future research.

Heaton (2003) outlines three key ethical and methodological issues to consider when employing secondary analysis of qualitative data;

I. Compatibility of the data with secondary analysis: is the data amenable to secondary analysis? This depends on the 'fit' between the purpose of the analysis and the nature of the original data. The purpose of the original data was to assess the effectiveness of family therapy vs. treatment as usual for selfharm, and the recordings are of the family therapy sessions. The SHIFT manual states that the therapist must explore the issues of self-harm and the adolescents were aware that they were attending the therapy sessions because they had harmed themselves. Therefore we can assume that when they are talking about their experiences they have a relationship to self-harm.

II. Position of the secondary analyst: ideally the analyst should have been part of the original research team in order to access original data, including tapes. Although I was not part of the original research team I obtained access to recordings of the Family Therapy sessions and the academic co-supervisor of this project was the lead investigator on the SHIFT trial and I was able to consult with them.

III. Reporting of original and secondary data analysis: it is important that the study design, methods and issues are reported in full, including an outline of the 
original study. The SHIFT trial (Wright-Hughes et al, 2015) is described in more detail within this report.

\section{$\underline{\text { Analytical approach }}$}

One method of analysis I considered was the Leeds Attributional Coding System (LACS; Stratton et al, 1988, cited in Stratton \& Bromley, 1999). LACS is a research technique which recognises and utilises the potential insights and benefits available through the study of people's social explanations in the form of attributions. The technique focuses on the attributions made by family members which include the explanations they give for everyday events which they experience. LACS provides a standardised method for coding and classifying attributional statements along five dimensions; stable or unstable, global or specific, internal or external, personal or universal and controllable or uncontrollable (Stratton \& Bromley, 1999). I thought that ascribing responses to pre-existing codes would be too constraining, as I was interested more broadly in exploring how adolescents understood their self-harming experiences, and I wanted to capture a range of responses. Therefore, I ruled out using LACS as a method of analysis.

I also contemplated using Discourse Analysis (DA) to approach the data. Gee (2014) states that while there are many different approaches, DA is the study of language in use. Some approaches focus on the content of the language being used, whereas other approaches focus more on the structure of language and how this structure functions to make meaning in specific contexts (Gee, 2014).

Gee (2014) describes discourse as the sequence of sentences and the ways in which sentences connect and relate to each other across time, in speech or writing. Braun and Clarke (2013) suggest that the analytic focus in DA is on understanding, in different ways, what language does, what realities are created through language, and how this occurs. Braun and Clarke outline two approaches to DA. Poststructuralist DA focuses on the content of language, but also the maintenance and disruption of power relations. Repertoire analysis focuses on both the content of language but also the use of language to construct certain social realities or psychological states. Although I am interested in the content of language in relation to self-harm, I will not focus on the way language, in relation to self-harm, is constructed. Also, with a 
secondary analysis, I would not have been able to achieve depth to the data sufficient enough to fully utilise DA. Therefore, I decided against using this approach.

One approach, described as a foundational method for qualitative analysis, is Thematic Analysis (TA), as outlined by Braun and Clarke (2006). TA is a method for identifying, analysing and reporting patterns (themes) within data. A theme captures something important about the data in relation to the research question, and represents some level of patterned response or meaning unit within the data set. A meaning unit is thought of as words, sentences or paragraphs containing aspects related to each other through their content and context (Graneheim \& Lundman, 2004).

Braun and Clarke (2006) suggest that in contrast to other qualitative approaches (such as Interpretative Phenomenological Analysis, IPA, or Grounded Theory) thematic analysis is not wedded to any pre-existing theoretical framework. They propose that TA can be a realist method, which reports meanings, experiences and the reality of the participants or it can be a constructionist method, which explores the ways in which events, realities, meanings, experiences etc. are constructed in a range of discourses in society. Similarly, they postulate that TA can be a contextualist method, which sits between the realist and constructionist methods, and acknowledges the ways in which individuals make sense of their experiences whilst recognising the ways in which the broader social context impinges on those meanings.

As I wanted to explore how participants understood their experiences of self-harm, whilst recognising that the social context has an impact upon how people make sense of their experiences, I felt that the contextualist approach offered a balance; as a method working both to reflect 'reality' and to unpick the surface of 'reality'. TA offers a flexible and useful research tool which can potentially provide a rich and detailed account of the data and allows a contextualist method to be used (Braun \& Clarke, 2006).

Themes, or patterns within data, can be identified in one of two ways in TA: in a deductive, or 'top-down' way, or in an inductive, or 'bottom-up' way. The deductive approach would be driven by the researcher's theoretical or analytic interest in the area. With this approach, the researcher is able to code data with a specific research question in mind, or the specific research question can evolve through the coding process (Braun \& Clarke, 2006). 
Initially, I considered the use of the deductive approach with Framework Analysis (Dixon-Woods, 2011) as a method to organise the data. Framework Analysis is a matrix-based method involving the construction of thematic categories into which data can be coded. This approach would have allowed me to code data to specific coding categories identified prior to the analysis, for example informed by existing literature, and then combined with other themes which are identified by subjecting the data to inductive analysis (Dixon-Woods, 2011). The intention would then be to see how the data fitted with established models for understanding self-harm.

As I began the process of familiarising myself with the data it became apparent that this approach was not suitable as I identified a range of responses related to self-harm and I wanted to capture this variety without the restriction of a theoretical framework with which to organise the data. As a result I utilised an inductive, or 'bottom-up', approach to analysis.

With an inductive approach, the themes identified should be strongly linked to the data, and the themes may bear little relation to the specific questions that were asked of the participants. They would not be driven by the researcher's theoretical interest in an area. It is therefore a process of coding the data without trying to fit it into a preexisting coding frame (Braun \& Clarke, 2006).

\section{$\underline{\text { Study context }}$}

This study involved secondary analysis of a subset of the recorded family therapy sessions from the SHIFT (Self-harm intervention: Family Therapy) trial. This was a multi-centre, individually randomised, controlled trial comparing Family Therapy (FT) with Treatment as usual (TAU) for adolescents aged 11-17 who had engaged in at least two episodes of self-harm (Wright-Hughes et al, 2015).

The primary objective of the trial was to assess the effectiveness of FT compared to TAU as measured by rates of recurrence of self-harm leading to hospital attendance eighteen months after randomisation.

Participants were randomly allocated to receive either FT or TAU. Those allocated to the FT treatment arm attended family therapy sessions, approximately $1 \frac{1 / 4}{4}$ hours duration, delivered over six months at roughly monthly intervals, totalling eight sessions. FT was delivered by qualified, registered, Family Therapists, working in 
teams of three to four and providing trial FT for a cluster of services. Within this approach, reflecting teams were used in the therapy sessions. This involved two, or three, members of the team observing the session through a one-way mirror. Provided that the family were interested in hearing their reflections, they would be invited in by the therapist. The reflecting team process invites the sharing of multiple personal meanings in a therapeutic context. Team members are encouraged to express their impressions and ideas (Haley, 2002). The practice is based on the idea that multiple views of clients' dilemmas offer hope of obtaining fresh perspectives of the situation through exposure to these various perspectives, so that clients may alter their perceptions, which in turn facilitates change (Jenkins, 1996, cited in Haley, 2002).

A modified version of the Leeds Family Therapy and Research Centre Systemic Family Therapy (LFTRC) manual (Wright-Hughes et al 2015) was delivered. FT sessions were recorded only where consent for this had been obtained from all family members present, with consent being obtained once from each family member for the duration of therapy. Recorded sessions were saved in an encrypted format onto appropriate movable media prior to transfer between NHS trust sites and the University of Leeds (Wright-Hughes et al 2015).

\section{$\underline{\text { Population }}$}

Adolescents were eligible for the SHIFT trial if they;

- Were aged 11-17 (from the date of their $11^{\text {th }}$ birthday to the day prior to their $18^{\text {th }}$ birthday);

- Had engaged in at least two episodes of self-harm (self-injury, self-poisoning, or both);

- Had been assessed in hospital following current episode, or referred directly to Child and Adolescent Mental Health Services (CAMHS) from Primary Care with recent self-harm as a key feature of presentation;

- Where living with their primary care-giver - both had to have given informed written consent where appropriate.

Where the presenting episode was due to alcohol or recreational drugs, the adolescent needed to have explicitly stated he/she was intending self-harm by use of alcohol/recreational drugs. 
Adolescents were not eligible for the trial based on the following;

- At serious risk of suicide;

- An on-going child protection investigation within the family;

- Would ordinarily be treated by a specific service and not CAMHS (such as psychiatric inpatient care);

- Pregnant at the time of trial entry;

- Actively being treated in CAMHS;

- In a children's home or short-term foster placement;

- Moderate to severe learning disability or lacked capacity to comply with trial requirements;

- Involved in another research project at the time of trial entry, or the previous six months;

- Siblings had been randomised to the SHIFT trial or were receiving family therapy within CAMHS;

- The young person and one main care giver had insufficient English language ability to contribute to data collection.

Following admission to Hospital/Accident and Emergency, or referred directly to CAMHS from Primary Care, adolescents were assessed for trial eligibility at their first presentation to CAMHS. When a young person met the criteria the CAMHS clinician introduced the trial at the assessment appointment. Those who provided consent were then contacted by a researcher who visited the family at home. Those who consented to participate in the trial were administered baseline assessments.

Where appropriate consent was obtained, and baseline assessments administered, participants were then randomly allocated to receive either FT or TAU via an automated system. A stratified random sample ensured that treatment arms were well balanced for the following; centre (CAMHS team), gender, age (11-14 or 15-17), living arrangements (with parents or guardians/foster care), number of previous episodes of self-harm ( 2 or $>3$ ) and type of self-harm (self-poisoning, self-injury, or combination). 
While 415 participants were randomly allocated to the FT arm of the SHIFT trial, 269 of these participants consented to the use of their session recordings to be used for future research (L Graham, personal communication, August 13, 2015). This constituted the potential data set in the current study.

\section{Current study}

\section{$\underline{\text { Ethics }}$}

Ethical approval was sought and granted from the National Health Service integrated research application system (see appendix 1). As this research was a secondary analysis of data previously gathered, and there was no contact with service users, ethical approval was granted through proportionate review. The protocol from this study has been approved by the SHIFT trial management team as applicable use of the data under the terms of the original participant consent. This project did not require local NHS research governance review (see appendix 2).

\section{$\underline{\text { Ethical considerations }}$}

Concerns regarding informed consent: informed consent was obtained during several stages of the SHIFT trial. Where a young person met the criteria, the assessing CAMHS clinician discussed trial participation at the assessment appointment, passed on the participant information sheet and requested consent for subsequent contact by a researcher. Those providing such consent were contacted by the researcher, who arranged to visit the family at home, and then obtained consent for participation in the trial (Wright-Hughes et al, 2015).

The participants randomly allocated to the family therapy treatment arm of the study, who consented to the sessions being recorded, were also asked to provide consent for their data to be used in relevant future research projects. Those that gave consent form the population of interest in this study.

Concerns related to data protection: the data is stored securely by the SHIFT management team based in the Clinical Trials Research Unit at the Leeds Institute of Clinical Trials Research. Although I accessed the data in the Leeds Institute of Health Sciences, I viewed the session recordings securely on nominated equipment. I was only able to access data for 10 participants at any one time. 
Family therapy session recordings were viewed and data identified as relevant was transcribed in a word processor for further analysis and then stored on the secure University m:drive (encrypted personal university IT account). The m:drive was accessed securely via Citrix if I was working remotely.

Concerns related to confidentiality: I did not have any access to personal details of the participants. I numbered participants differently to how they were recorded by the SHIFT management team and recorded participants' age by range rather than specific year. Any quotations from participants included in the final report were reported anonymously. These measures were taken to reduce the likelihood that participants could be identified.

\section{$\underline{\text { Participants }}$}

The sample in the present study consisted of a subset of those participants in the SHIFT trial recruited to the FT treatment arm of the trial. Within qualitative research sample size is a disputed topic. In a review paper for the National Centre for Research Methods (NCRM), Baker and Edwards (2012) approached fourteen renowned social scientists and five early career researchers and proposed the question "how many interviews are enough?" Based on the responses, Baker and Edwards concluded 'it depends'. There are a number of factors to consider, for example the author's theoretical perspective (for example that there is an 'objective truth' to be discovered) and methodological factors such as the diversity of the sample; a particularly heterogeneous sample is likely to increase the sample size whereas if the population is reasonably homogeneous then a smaller sample size will capture the diversity required (Ritchie \& Lewis, 2003). Similarly, the larger the selection criteria, the larger the sample. The type of data collection method will influence sample size (for instance single interviews, paired interviews, group interviews), as will practical constraints, such as time (Ritchie \& Lewis, 2003)

In order to determine the sample size for this study I used the concept of data saturation. This has become 'gold standard' in health science research, which can be thought of as the point in data collection and analysis when new information produces little or no change in the themes identified (Guest, Bunce \& Johnson, 2006). These considerations of saturation have been thought of mainly in relation to primary data collection. In this study saturation was achieved when data collection and analyses 
produced little or no change in the themes of self-harm identified. I thought that the sample would likely include larger numbers of participants than is typical for qualitative research as an individual's data may not have been rich in detail. I was looking to capture a range of responses as I was not conducting an interview in which I would have been able to follow up an initial response with more questions to uncover further information, and ensure depth to the data from one participant. Similarly, it was likely to be the case that not all of the session material was relevant to the research question.

Polkinghorne (2005) states that participants are selected for a qualitative study because they can provide substantial contributions to filling out the structure and character of the experience under investigation. As I was looking to canvas a wide range of views and experiences on the topic of self-harm, across the sample of adolescents involved in the SHIFT trial, I used a sampling frame based on a number of variables that hopefully afforded maximum variation in the responses. The sampling frame was guided by the information captured on the SHIFT trial screening form and the variables of interest in this study were; age (across the age range of participants' included in the study; 11-17 - half 11-14 \& half 15-17) and gender (including roughly equal numbers of both males and females).

I only included those participants who had a minimum of three Family Therapy sessions recorded. This was selected as an inclusion criterion as it was felt that the first sessions were likely to be general introduction and familiarisation with the approach - resulting in fewer conversations about self-harm - and because some participants only attended one family therapy session and then disengaged from the intervention. Therefore, by selecting participants who attended a minimum of three sessions, I assumed that the depth of data with regards to self-harm was available.

\section{$\underline{\text { Data collection }}$}

Initially session recordings from ten participants, who met the minimum criteria outlined above, were randomly selected by a SHIFT trial administrator, and uploaded to a secure folder on the m:drive and made accessible. I selected the first participant (the folders were in numerical order) and began the process of familiarising myself with the data by watching the first session. 
I watched each session recording within the participant's folder where the adolescent was present and discounted those sessions where the adolescent was not (such as parent only). Initially, I set out with the intention of only transcribing data that the adolescent contributed to that related directly to self-harm. The subject of self-harm was often discussed in the first session and instigated by the therapist, as they would explain the purpose of the SHIFT trial and usually ask if self-harm was still happening. I noted down the first mention of self-harm for each participant, who instigated this, and the context of this. I only transcribed data for later analysis if the adolescent was directly involved in this conversation. For example, if the parent $\&$ therapist were discussing self-harm or seemingly relevant contextual information, and the adolescent did not contribute, this data was not captured. Similarly, I did not transcribe any data from reflecting team conversations as the adolescent would be invited to listen to these conversations and offer their own reflections once these conversations had finished.

Along with capturing data directly related to self-harm, as described by the adolescent, it also became apparent that I needed to capture data which contextualised self-harm. The adolescents described a range of difficult life experiences which may have contributed to the development of self-harming behaviour and it seemed important to highlight this. For example, one adolescent described self-harming because they believed they deserved to be punished. However, this was in the context of growing up and experiencing several years of emotional and physical abuse. By capturing data, from the adolescent, related to this relationship, I was able to 'set the scene' and comment on life experiences which may have contributed to the development and maintenance of self-harming behaviour. Therefore, if contextual information was provided in earlier sessions, I would look out for this in later discussions and transcribe the relevant data. If this contextual information was provided in later sessions I would revisit the earlier sessions and transcribe the relevant data.

I watched all of the sessions for each participant and transcribed data I deemed relevant. For example, if an adolescent described harming themselves in response to conflict with a friend I would transcribe this data. I would then also transcribe related data in future sessions. The same process was used for each participant.

I also noted down key themes discussed in each session. This allowed me to revisit earlier recordings to capture data. For example, if during one session the adolescent described self-harming in response to family conflict, I would select and transcribe 
data from the sessions where the adolescent was discussing family conflict. If, during further sessions, the adolescent discussed their self-harm in relation to another topic (such as bullying) I would revisit earlier sessions and capture data related to the other topic as well.

After watching, and transcribing, the entire number of sessions - where the adolescent was present - for the first ten participants I then completed a sampling framework. I used the framework to capture demographic information for each participant; age range, gender and ethnicity. This then allowed me to request, from the SHIFT management administrator, participants whose characteristics would ensure I captured variation across the framework. For example, if the framework highlighted lower numbers of male participants, or participants of a particular age range, I would ask the administrator to include those participants in the next ten that I was able to access.

\section{Analysis of the data}

While there is no clear agreement about what thematic analysis is, or how to go about it, Braun \& Clarke (2006) helpfully devised a checklist of criteria to consider when determining whether you have generated a good thematic analysis during the transcription, coding, analysing and writing up stages:

Table 1: A checklist for achieving good TA (Braun \& Clarke, 2006)

\begin{tabular}{lll}
\hline Process & No. & Criteria \\
\hline Transcription & 1 & $\begin{array}{l}\text { The data has been transcribed to an appropriate level of } \\
\text { detail, and the transcripts have been checked against the } \\
\text { tapes for 'accuracy'. }\end{array}$ \\
Coding & 2 & $\begin{array}{l}\text { Each data item has been given equal attention in the coding } \\
\text { process. } \\
\text { Themes have not been generated from a few vivid examples } \\
\text { (an anecdotal approach), but instead the coding process has } \\
\text { been thorough, inclusive and comprehensive. } \\
\end{array}$ \\
& & All relevant extracts for each theme have been collated.
\end{tabular}


5 Themes have been checked against each other and back to the original data set.

6 Themes are internally coherent, consistent, and distinctive.

Analysis $\quad 7 \quad$ Data has been analysed - interpreted, made sense of - rather than just paraphrased or described.

8 Analysis and data match each other - the extracts illustrate the analytic claims.

$9 \quad$ Analysis tells a convincing and well-organized story about the data and topic.

10 A good balance between analytic narrative and illustrative extracts is provided.

Overall 11 Enough time has been allocated to complete all phases of the analysis adequately, without rushing a phase or giving it a once-over-lightly.

Written report 12 The assumptions about, and specific approach to, thematic analysis are clearly explicated.

13 There is a good fit between what you claim you do, and what you show you have done - for example described method and reported analysis are consistent.

14 The language and concepts used in the report are consistent with the epistemological position of the analysis.

15 The researcher is positioned as active in the research process; themes do not just 'emerge'.

The same authors produced a guide of how to operationalise the checklist through sixphases of analysis;

1. Familiarising oneself with the data - transcribing data (if required), reading and re-reading the data, noting down initial ideas. 
2. Generating initial codes - coding interesting features of the data in a systematic manner across the entire data set, collating data relevant to each code.

3. Searching for themes - collating codes into potential themes, gathering all data relevant to each potential theme.

4. Reviewing themes - checking if the themes work in relation to the coded extracts (level 1) and the entire data set (level 2), generating a thematic 'map' of the analysis.

5. Defining and naming themes - On-going analysis to refine the specifics of each theme, and the overall story the analysis tells, generating clear definitions and names for each theme.

6. Producing the report - the final opportunity for analysis. Selection of compelling extract examples, final analysis of selected extracts, relating back the analysis to the research question and literature, producing scholarly report of the analysis.

In this project, phases one and two formed a hybrid process as I was familiarising myself with the data and watching session recordings, whilst simultaneously selecting interesting features of the data to transcribe for later analysis. Data was listened to in an active way, to search for patterns and meanings within the data. I used a field diary to make a note of my initial reaction to session recordings which I was able to revisit during the coding stage.

The selected data was transcribed into a word processing document. For the analysis, I used NVIVO 11, a qualitative data analysis computer software package. Next I reread each transcript and generated initial codes. Codes consisted of meaningful units of text, ranging from a single word to a paragraph. In some instances, a piece of text was coded more than once, if I thought it held separate meanings. With NVIVO I was able to select and add data to existing codes, or I could create a new code. Once I had coded each transcript fully I moved on to the next (see appendix 3 for examples of the analysis in NVIVO).

After all of the transcripts had been fully coded I moved through to phases three and four. This involved me grouping the generated codes into potential themes. This was a process I moved between and back and forth several times, having re-read potential themes and deciding the codes did not quite fit, or the theme did not accurately reflect 
the codes. Using NVIVO I was able to create a 'thematic map' which allowed me to 'step-back' from the data on occasions to see the overall picture.

Phase five involved me grouping codes together into potential themes. I named each theme and having named potential themes I would then meet with my main project supervisor and co-supervisor who reviewed the potential themes and checked to see if the themes worked in relation to the codes I had developed. The written analysis (phase six), included selected extracts to support the codes and themes. These were also reviewed by, and discussed with, my supervisors. This process was refined over several editions of the analysis.

\section{Quality checks}

Tobin and Begley (2004) proposed the need establish the legitimacy of the qualitative research process. Lincoln and Guba (1985, cited in Tobin \& Begley, 2004) use the term 'trustworthiness' which is demonstrated through credibility, transferability, dependability and confirmability.

Credibility addresses the issue of 'fit' between participants' responses and the researcher's interpretation of them, and whether it is credible (Schwandt, 2001 cited in Tobin \& Begley, 2004). This has been demonstrated with the use of audit trails (for instance ensuring that the process of research is logical and clearly documented). I held discussions with my supervisors, experienced researchers, around identifying and refining themes that emerged from the data.

Transferability refers to the extent to which the findings can be transferred to other groups or settings (Polit \& Hungler, 1999, cited in Graneheim \& Lundman, 2004). I have facilitated transferability in this research by including a description of the selection criteria and characteristics of the participants, as well as detailing the data collection and analysis process.

Dependability is achieved through a process of auditing (Tobin \& Begley, 2004). This allows others to examine my documentation of the data, methods, decisions and final product. Key to this is the concept of 'reflexivity' - a self-critical account of the research process. I used a journal throughout the process to make notes of my initial reactions to watching the session recordings. I have also commented on my position, beliefs and experiences in the research (see below). 
Confirmability is concerned with establishing that data and interpretations of the findings are clearly derived from the data (Tobin \& Begley, 2004). Polkinghorne (2005) states that excerpts from the data should illustrate the findings and show the reader how the findings were derived from the data. I have included quotes verbatim from participants in support of the themes I have constructed.

\section{$\underline{\text { Researcher statement }}$}

Braun and Clarke (2013) suggest that reflexivity is an essential requirement of good qualitative research and a quality control. They refer to reflexivity as the process of critically reflecting on the knowledge we produce and our role in the knowledge. Personal reflexivity brings the researcher into the research and makes them visible as part of the research process. This process considers how the assumptions and experiences of the researcher can influence the production of knowledge (Braun \& Clarke, 2013).

I am a 29 year-old white male training to be a Clinical Psychologist. I was in part motivated by the research topic through clinical experiences when working as an Assistant Psychologist in a Child and Adolescent Mental Health service (CAMHS). An encounter with a young person, who was self-harming prolifically, left me wondering how this young person understood their self-harm. I feel this motivated me to want to understand individual experiences of self-harm, and I wanted the opportunity to listen to the stories adolescents told, in their own words.

I assumed that some adolescents would find it difficult, based on my own experiences of working clinically, and was surprised when watching the recordings at how open some young people were when discussing self-harm. I felt privileged to have heard the perspectives of adolescents talking about self-harm, and how they made sense of this.

I was aware when watching the session recordings and analysing the data that I was taking a different perspective; a researcher as opposed to a trainee Clinical Psychologist. Documenting my responses to recordings, along with discussions in supervision, contributed to my reflexivity and an acknowledgement of my role in the analysis and interpretation. 


\section{CHAPTER THREE: RESULTS}

\section{$\underline{\text { Summary of participants }}$}

I viewed each session recording for 22 participants, watching a total of 113 sessions. As stated in the method chapter, one inclusion criterion for this study was a minimum of 3 session recordings for each participant. In each of the participant folders uploaded by the SHIFT management team - were a minimum of 3 session recordings. However, in some instances these included parent only sessions. Therefore, the number of session recordings per participant, where the adolescent was present, ranged from 1 to 12 , with a mean of 5.1 sessions per participant. Approximately 170 hours of footage formed the dataset.

Of the 22 participants, 14 were female $(63 \%)$ and 8 were male (37\%). Twelve participants were aged between 11 and 14 (55\%) and ten were aged between 15 and 17 (45\%). Participants were mostly white (77\%), with the remaining sample Asian (9\%), Mixed Race (9\%) and Black (5\%).

All participants had harmed themselves on at least two occasions (a criterion of the original SHIFT trial) and many had harmed themselves on numerous occasions. Multiple methods of self-harm were described including cutting, scratching, biting, punching, poisoning and overdosing. Six adolescents described using a number of methods to harm themselves across different episodes. Two adolescents used more than one method of self-harm within the same incident.

The topic of self-harm was introduced in the first session in 18 of the $22(81 \%)$ cases, and in most instances this was by the therapist (77\%). In a minority of sessions the adolescent or parent brought it up. Table 2 displays the participant demographic information. 
Table 2: Summary of participants

\begin{tabular}{|c|c|c|c|c|c|}
\hline Participant & Sex & $\begin{array}{c}\text { Age } \\
\text { Group }\end{array}$ & Ethnicity & Method & $\begin{array}{c}\text { No of sessions } \\
\text { (adolescent present) }\end{array}$ \\
\hline 1 & Female & $15-17$ & White & Not known & 2 \\
\hline 2 & Female & $11-14$ & White & Cutting & 11 \\
\hline 3 & Female & $11-14$ & White & Cutting & 1 \\
\hline 4 & Female & $11-14$ & White & Multiple & 8 \\
\hline 5 & Female & $15-17$ & Black & Multiple & 6 \\
\hline 6 & Male & $11-14$ & Asian & Not known & 3 \\
\hline 7 & Female & $15-17$ & White & Overdose & 4 \\
\hline 8 & Female & $15-17$ & White & Cutting & 7 \\
\hline 9 & Female & $11-14$ & White & Overdose & 5 \\
\hline 10 & Female & $11-14$ & White & Cutting & 7 \\
\hline 11 & Male & $11-14$ & Mixed & Scratching & 1 \\
\hline 12 & Female & $15-17$ & White & Multiple & 6 \\
\hline 13 & Male & $15-17$ & White & Cutting & 4 \\
\hline 14 & Male & $15-17$ & White & Cutting & 11 \\
\hline 15 & Male & $11-14$ & White & Punching & 1 \\
\hline 16 & Female & $11-14$ & White & Multiple & 6 \\
\hline 17 & Female & $11-14$ & Mixed & Multiple & 5 \\
\hline 18 & Male & $15-17$ & White & Punching & 4 \\
\hline 19 & Female & $11-14$ & White & Multiple & 6 \\
\hline 20 & Female & $11-14$ & White & Overdose & 3 \\
\hline 21 & Male & $15-17$ & White & Multiple & 3 \\
\hline 22 & Male & $15-17$ & Asian & Multiple & 9 \\
\hline
\end{tabular}

I have used the following key to refer to individuals included in the illustrative quotes:

$\mathrm{T}=$ Therapist

$\mathrm{FM}=$ Family member

$\mathrm{P}=$ Participant 
If no initial accompanies the quote then it will be directly from an adolescent. Family members involved in the sessions included mothers, fathers, siblings and grandparents. In some instances I have provided the speech of a family member in order to provide context for the adolescent's quote.

\section{$\underline{\text { Setting the scene }}$}

During the data collection phase, it became apparent that in order to contextualise the data related to self-harm, it was important to reflect some of the difficult life experiences that the adolescents described, many of which may have played a role in the development and maintenance of self-harm later in their lives.

Whilst the adolescent was the referred individual, on the basis of having harmed themselves, the family therapy approach works with the family as a system and sessions generally contained multiple members of a family. Strained, or fractured, family relationships were evident within the sessions. Often examples of conflict between therapy sessions were highlighted although heated exchanges and arguments between the adolescents and their parents, and/or siblings, were frequent in the sessions. In some instances there had been a complete breakdown of a relationship resulting in the adolescent moving out of the family home and residing with members of their extended family or grandparents.

Accounts of abuse were present in some of the sessions. Mainly this related to experiences of physical and emotional abuse, although sexual abuse was reported by a participant. They did not disclose who the perpetrator of the abuse was but described "being raped" as a teenager.

In one conversation, an adolescent's parent talked of suffering years of physical abuse and being beaten by their partner. The adolescent spoke about witnessing and hearing the physical abuse. This parent and adolescent recalled an argument between the two of them which had escalated into physical violence and the parent had struck the adolescent. Another participant remembered an incident of being hit in the face by their parent's partner. They recalled the incident taking place following an argument, although in the session this was disputed by their parent.

Emotional abuse was described by some adolescents in the sessions. For example, bullying was reported, often by peers, and in one instance this was racially motivated. 
A participant described experiencing several years of emotional abuse from their stepparent. They provided numerous examples of being treated very differently from their step-siblings and the individual's biological children. They were verbally abused on a regular basis and attributed the treatment they received in this relationship to "ruining" their life:

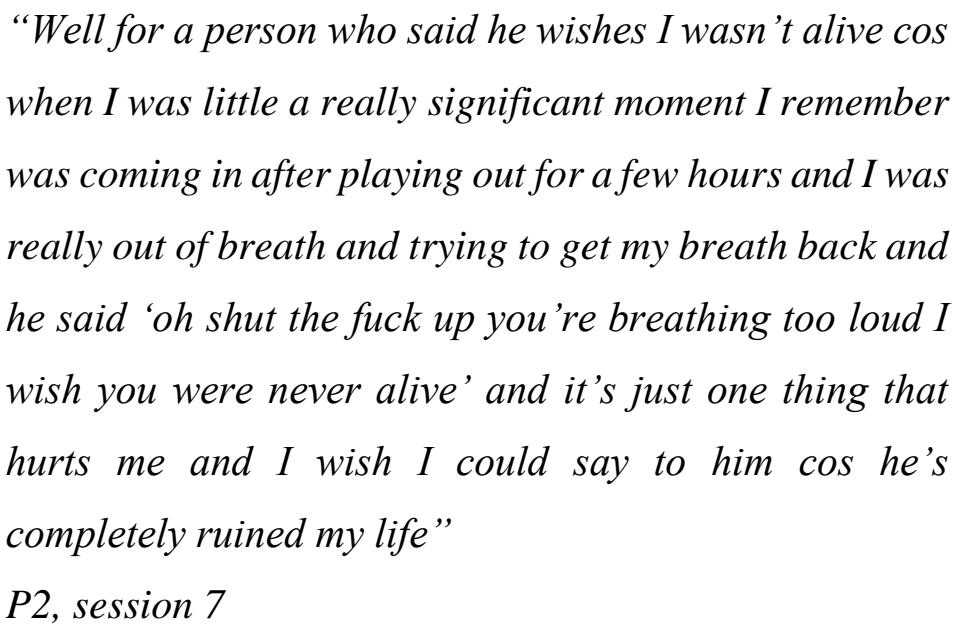

Another aspect of chaotic and disruptive lives reported by participants was substance use. For example a participant described using hard drugs at a very young age with friends. For others this was alcohol use. Consequences included fractured relationships with their parents, or a reduced capacity to manage a situation. In one instance alcohol was a factor in self-harming behaviour:

\footnotetext{
“Yeah I wouldn't cut if I've only had one or two I've only ever done it if I've been really drunk but then I've also been really drunk and not done anything stupid" P14, session 3
}

\section{$\underline{\text { Core themes }}$}

Five core themes were derived from the data. These were; (1) Distress can be difficult to convey (2) Self-harm and suicidal ideation; a complex relationship (3) Self-harm as a form of communication (4) Self-harm to manage emotions and (5) Moving forward. Figure 1 displays the five core themes generated from the thematic analysis, along with sub-themes. Each of the themes and sub-themes will be described in detail in this chapter and will contain participant quotes to illustrate the analytic claims. 
Figure 1: Thematic map

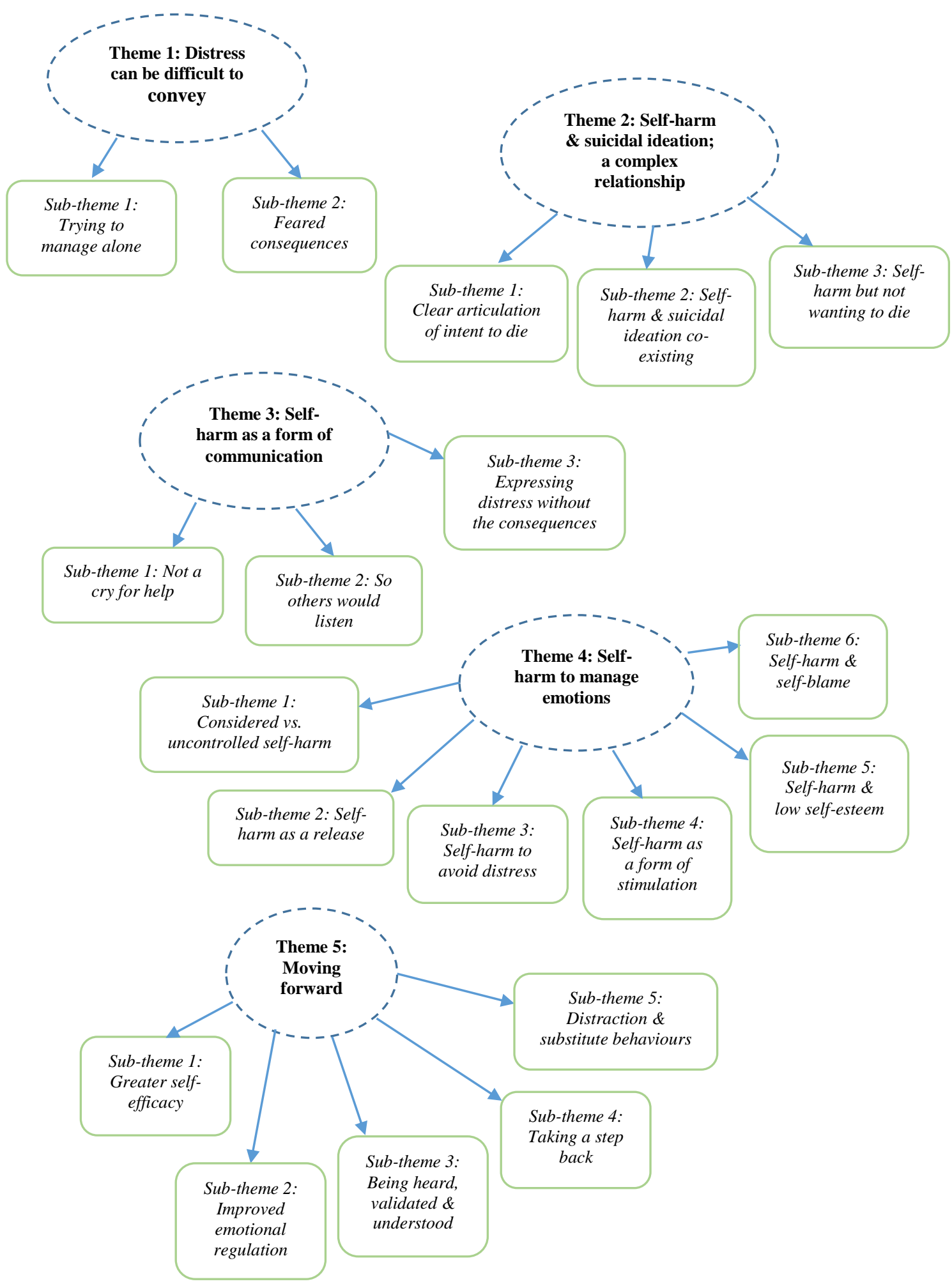




\section{Theme 1: Distress can be difficult to convey}

Given the context of the sessions and therapeutic modality, content often focussed on how the family as a system communicated, both inside and outside of the sessions. However, conversations also frequently explored specifically how the adolescents communicated. A number of participants found it very difficult to explore their distress, or even broach the subject of self-harm. For example, when posed a question, some adolescents managed only limited verbal responses, replying with "don't know", "can't remember" or "not sure". It's likely that with all of these replies there were elements of the content of the questions being difficult to address in the therapy setting, but also genuinely not knowing the answer. For example, when asked their thoughts on a recent episode of self-harm, one adolescent commented "I'm confused by all of this". It may also have been the case that individuals did not have the words to explain, were not able to remember, or perhaps did not want to remember.

\section{Sub-theme 1: Trying to manage alone}

Others were able to articulate why they found communicating difficult and a number of reasons were provided explaining why adolescents struggled to talk both inside and outside of the therapy sessions. Often they were trying to manage alone by bottling their feelings up and not sharing their thoughts with anyone. One participant reflected they "should" manage in this way while in the extract below a participant comments that they let their feelings "bottle up" however this then resulted in an eruption:

\section{"I just let it bottle up...bottle up then just exploding basically” \\ P12, session 2}

\section{Sub-theme 2: Feared consequences}

The impact, or feared impact, of sharing their thoughts and worries prevented some adolescents from opening up and talking to those around them. Not wanting to burden other people with their worries, or wishing to protect loved ones from harm, caused individuals to keep quiet. In the quote below, a participant describes feeling pressured into talking and this not being helpful. They describe a complex mix of self-blame for not talking in the first place - coupled with feelings of regret for revealing things that had then caused upset: 
"Well yeah because I would probably say more than I wanted because I've been pushed to say it and stand up and be like well are you happy now well nobody is happy now and I'd be feeling even worse because I didn't say it in the first place because I didn't want to upset them and I'd feel it's my fault not what I've said but because I've said it and now they're upset and its then caused a problem and it feels like either way I don't win"

P5, session 6

The fear of being of judged was also a barrier to communication. For example, a participant described not feeling able to talk to their parent or grandparent when they were feeling very low, and suicidal, as they were scared that their grand-parent would tell other people. In the example below, another participant explains how being asked to discuss self-harm made them feel judged and this was incredibly distressing, to the extent that they left the therapy session to avoid the distress:

\footnotetext{
“Makes me feel like I'm being judged and I don't like that so I'm gonna go before I cry even more and before I have a breakdown" $P 1$, session 2
}

Some adolescents recalled negative reactions from family and friends having talked about self-harm. In the example below, a participant explains how they were viewed as "mad". Despite not wanting to tell their family about self-harm, they found out and reacted with a stereotypical response which the adolescent found extremely upsetting:

T: "What was it like telling your family?"

A: "Horrible, but I didn't really tell them, they found out and I had to deal with them calling me a nutcase and it wasn't very nice at all"

P8, session 1 
This negative view of self-harm was internalised by some participants. One adolescent described how when they were experiencing low mood self-harm was a way in which to stimulate feelings, and they reflected on this behaviour as "stupid" and irrational. In the example below, a participant recalls how they had reached the conclusion that self-harm was unwise:

"Because I realised what a stupid thing it was to do" P17, session 1

\section{Theme 2: Self-harm \& suicidal ideation: a complex relationship}

This theme reflects the complex relationship between self-harm and suicidal ideation. As will be detailed in themes three and four, there were times when the adolescents were able to describe why they had harmed themselves. While some adolescents described being unsure of their motivation others clearly articulated intent to end their life and complete suicide when recalling episodes of self-harm. There were examples of thoughts of self-harm and thoughts of wanting to die being more complicated, and described as existing during the same episode of self-harm. There were also instances of self-harm being unrelated to suicidal intent.

\section{Sub-theme 1: Clear articulation of intent to die}

A number of participants reported attempts to complete suicide. For some there had been multiple attempts. Methods of attempted suicide varied across and within episodes. For example a participant recalled how, on their one and only attempt, they had tried to overdose, then poison themselves and would have gone on to cut their wrists if they had not been interrupted.

While thoughts of wanting to die fluctuated, one adolescent reported that they experienced thoughts of wanting to die each day irrespective of their mood. Another explained how they heard voices that told them to give up and stop trying. This experience made them "sick of life" and they had thoughts of wanting to end their life as a result. Whilst these thoughts distressed them, they explained how on occasions they actually wanted these thoughts to come. In the extract below, a participant described a time when they were feeling incredibly angry, and had thoughts that life was not worth living. They went to their bedroom and took a number of tablets with 
the intent to die. Throughout their therapy sessions, this participant shared their belief that they had been they had been 'set-up' and treated unfairly, or unjustly, from a young age. They explained how their life was not one they wanted to live and they perceived themselves to have no control either:

\section{A: "I went to my room and took about eight and waited" \\ T: "And did you have an idea what the pills might do?" \\ A: "I thought they might kill me" \\ P22, session 1}

A sense of rejection and abandonment contributed to a participant experiencing suicidal thoughts. In the quote below, the adolescent recalls how their parent had left them and they had relocated several times, resulting in them struggling to form relationships at school, all of which contributed to a sense of being abandoned by others:

"About three months, I went to school and were thinking 'nobody wants me here' and my grades were falling and that and I've had to move here and there and my mum left me and that I'd locked myself away in my bedroom and thought I could do it this way or that way"

P18, session 1

Self-blame was a factor for a participant attempting to complete suicide. They explained how they had reached the conclusion that they were to blame for all of the problems in their family and that their completed suicide would have actually benefitted their family. They described attempting to complete suicide by taking tablets, stating that they would have continued if they had not been interrupted by their family:

"I was sat thinking about times when things have gone wrong before and I was thinking it seems to be me that's the cause of anything that went wrong in the family and I thought it would be better if I wasn't here and I thought 
if I wasn't here they wouldn't have to deal with me and I just got loads of adrenaline and I wasn't even thinking and just went downstairs and spent the next three hours just doing it and I would have just kept going had they not found me"

P5, session 2

However even with intent to die, it was still complex. This individual explained how they had experienced relief after their attempt at completing suicide had been unsuccessful. They had taken several pills with the intent to die and believed they had taken enough pills to end their life. However later the same day they vomited and described experiencing both relief and disappointment; relief as they thought they could not have not done much harm to themselves, and disappointment as they would still have to face their problems that had contributed to them taking the overdose:

A: "I went to the bathroom and was sick"

T: "And were you disappointed with that?"

A: "Erm, a bit of both, I was happy because I thought that if you'd been sick that you couldn't do that much damage and disappointed because I still have to go back to school"

$P 4$, session 2

Whilst hopelessness was linked to suicidal ideation, and expressed through thoughts of giving up and a view that life was not worth living, this was not always acted on. For example, a participant described experiencing suicidal ideation "for a week" but did not take any action or make any attempt to end their life. In the quote provided, this individual describes ruminating on difficult life experiences and this leading to a catastrophic view that their future would be bleak with no prospect of this changing. While this process appeared to overwhelm the adolescent and they were unable to see a way forward, they had not acted on these thoughts:

"My mind was going into overload and I was thinking of all the crap things that had happened to me in my life and 
how crap my future was going to be so I thought "sod it

I don't want to be here anymore"

P2, session 6

\section{Sub-theme 2: Self-harm \& suicidal ideation co-existing}

Suicidal ideation and self-harm co-existed within, and across, episodes. Some participants described episodes of self-harm without wanting to die, yet reflected on other episodes of self-harm with intent to complete suicide. For example, this adolescent describes how on some occasions they had self-harmed to lose blood with the intention to die, yet at other times had self-harmed to draw blood as this had a calming effect for them:

A: "Yeah, I do it cos I want the blood... a lot of times I've done I've done it and thought 'right I don't wanna be here' so I cut it to try and lose as much blood as possible"

T: "To try and pass out?"

A: "Yeah that's been the reason I've don't it a few times but I've never passed out...but with them ones I've done it because I want some blood"

$P 2$, session 7

There were also examples when intent was less clear. This was often expressed by adolescents as wanting problems to disappear, or themselves to disappear. The idea that they should disappear was perhaps recognition that it was not possible for all of their problems to vanish so the alternative was that they no longer exist, and therefore not have to face the difficulties in their lives. For example, one individual described experiencing thoughts of "death" generally, but commented that they "didn't have the guts" to attempt to complete suicide. In this example, a participant, who at times said they had self-harmed with an intent to die and at other times had self-harmed with no intention to die, said that sometimes they simply wanted to escape their problems: 
“But I don't also think about self-harm sometimes I want to overdose or if I don't want to hurt myself I'll just want to run away and sometimes it's dead hard"

P10, session 2

In this extract, a participant explains that when they were harming themselves using "scarification" - which they described as "basically a tattoo done with scarring" - this was not related to thoughts of suicide and they wanted the resulting scars to be there. They explained how they had let a girlfriend carve their name into their back, almost as a way of memorialising this important relationship. They stated that they did not feel pain when being scarred and they were proud of the results, whereas their selfharm was related to angry thoughts or thoughts of not wanting to be alive:

"No it's different...when I'm doing it it's because I'm annoyed and genuinely don't wanna live and with this I can't feel the pain and I'm doing it because I want it to be there but with a lot of my self-harming stuff I don't want it to be there"

P2, session 7

\section{Sub-theme 3: Self-harm but not wanting to die}

There were also clear statements that indicated for some, self-harm was not about wanting to die. There were many instances of talk about self-harm that was not related to suicidal intent or thoughts. For example, in this excerpt, a participant explains how their self-harm became more severe although this was an attempt to manage their distress and not an attempt to end their life:

\footnotetext{
"Well yeah it's like I do it further down and a bit deeper and stuff but I don't want to die or anything I just want don't want to keep on getting upset" P4, session 1
}

In contrast to those individuals who expressed a clear intent to die, others described actively trying to stay alive despite experiencing suicidal ideation, which was 
characterised as a struggle. In the quote provided below, a participant describes trying to stay alive despite experiencing dark thoughts:

"Yeah because when the bad things comes the struggle is trying to keep yourself alive"

P7, session 1

Theme 3: Self-harm as a form of communication

As highlighted in theme one, distress was difficult for some individuals to discuss. While some participants may not have had the words to voice their distress, or not wanted to share their thoughts, the act of self-harm offered a means of communication. This was expressed in a number of ways. Self-harm described in this theme was not related to suicidal intent.

\section{Sub-theme 1: Not a cry for help}

There is a belief held in wider society that some people harm themselves "for attention". This is the idea than an individual would choose to self-harm so that others will take notice. This view, that the act of self-harm is to draw attention to oneself, was shared by family members of more than one participant when asked to reflect on the adolescent's self-harming behaviour. However, views expressed by adolescents suggest that, for some, self-harm is more nuanced than simply a means of drawing attention. For example, in one therapy session, a sibling commented that the only reason that they were attending family therapy sessions is because the adolescent had been "stupid" and self-harmed for attention. The adolescent replied that was not the case and reflected back to their sibling that they did not understand "at all". In the extract below, another participant reflects back to a parent that they understood why they had formed the view that self-harm was a "cry for help" but pointed out that they had not displayed their self-harm for others to see:

"I wouldn't say it was a cry for help although I can see why you've come to that conclusion...I mean I wasn't really parading around showing everyone” P8, session 1 


\section{Sub-theme 2: So others would listen}

In contrast to the paragraph above, there were examples of self-harm being utilised so that others would attend to them. However, the function was not simply to draw attention, but to communicate to others the level of distress and for people to recognise this distress, and then take action. Not being heard was stated as being important and linked to self-harm. In one example a parent suggested that the adolescent was at more risk of harming themselves when the two of them had argued. The adolescent's response suggested that while that could have been the case, their self-harm was also related to people not acknowledging them, or listening to them. In the extract below, another adolescent, who was experiencing a tough time at school, explains that cutting allowed them an opportunity to express to their family, and staff at the school, how distressing they found attending school. They hoped that self-harm would make the school staff notice and take action:

\section{T: "Can you think made you decide to cut yourself?" \\ A: "I knew that if I did that school would actually listen"}

P4, session 3

Participants reported that thoughts or acts of self-harm often followed conflict with family and/or friends. These included instances where the adolescents had been directly involved in or witnessed arguments. The act of self-harm appeared to offer individuals an opportunity to express how distressing they found conflict. In other instances, self-harm was used to not only express distress, but to try and put a stop to an argument. For example, a participant recalled a family disagreement from childhood, commenting that they had harmed themselves as a way of expressing how distressing they found this and wanted it to stop, hoping self-harm would make their family members "shut up":

\footnotetext{
T: "Did you say you were ten or eleven when you first self-harmed?"

FM: "Eleven"
} 
A: "I don't remember what it was about but I just remember an argument between members of the family and I just wanted them to shut up basically"

P14, session 8

\section{Sub-theme 3: Expressing distress without the consequences}

There is some overlap with this sub-theme and the content of theme four, self-harm to manage emotions, in that self-harm offered these participants a way of managing distress. However, the responses captured in this sub-theme suggest that self-harm also allowed these participants the opportunity to vent their frustrations without hurting others. They described not wanting to hurt other people and by harming themselves it protected others. For example, a participant described having had their mobile phone confiscated and then punching through a window; the glass became the target of their anger rather than the parent who had taken the phone from them. Although that action carried consequences, it was less than striking a parent would have been. The quote below highlights how an argument with a teacher left another adolescent feeling enraged, with thoughts of wanting to lash out at the teacher. However, instead of acting on this and incurring serious repercussions, they selfharmed as a way of releasing their anger:

"The real self-harming started because I was arguing with a teacher and instead of hitting him I hit a wall and that felt good for me so I were continuously punching walls and I were punching big thick metal bars and that lot just to get my anger out" P18, session 1

Similarly, a participant described conflict with their peers and being on the receiving end of bullying. Instead of retaliating verbally, for which they would have been reprimanded, they waited until they were home and then self-harmed. This allowed them the opportunity to express their frustration, which they experienced at the time they were being bullied, but without the consequences they would have faced if they had reacted: 
"I'll get in trouble and I know I've said things back but I didn't start any of it and they'll make up horrible songs about me and I'll say things back and then I'll get in trouble and then I'll get upset and say horrible things to the teachers and then I'll get in even more trouble and then I'll just do it when I get home"

P17, session 1

\section{Theme 4: Self-harm to manage emotions}

This theme, along with the sub-themes, reflects the role self-harm played in managing emotions. These responses were distinct from those related to self-harm and suicidal ideation. Self-harm was often a strategy employed to regain, or retain, control over emotions.

\section{Sub-theme 1: Considered vs. uncontrolled self-harm}

For some self-harm was pre-meditated and in these instances the adolescents often described their thoughts in the build-up that led to the act. This was described as a process of internal dialogue and different to the impulsive acts of self-harm. For example, a participant explained how they explored several options to manage their distress, although self-harm was the thought that they became fixated on, and the option that was selected. As opposed to mentally cycling through their options and ending up at self-harm, the adolescent in the excerpt below describes how when they were angry they did not think that they had another option available other than to selfharm:

"Sometimes I'm angry and I don't know what else to do" P10, session 3

The urge to self-harm was also characterised as unpredictable and compulsive. Some individuals harmed themselves impulsively, reporting that they "just did it" or needed to "get it done". In the example below, this adolescent describes self-harming but then not feeling able to stop: 
"Then one day I couldn't stop and started cutting and cutting and couldn't stop at all",

P19, session 1

In contrast to what was depicted as a calm internal conversation, as described in the first paragraph of this sub-theme, some individuals explained that they had little control over thoughts to self-harm, and were powerless. These thoughts were likened to external forces. For example, a participant explained that when they were angry they were like a "different person" with "different people inside" of them. Another participant portrayed the urge to self-harm as a raging battle between external forces of good and evil, and being powerless to the "evil" thoughts to self-harm:

\section{"It's like sometimes a devil just enters my head and the angel just gets pushed aside and it's like the exorcist if you get me like it just comes quickly and you can know it's going to happen but you can't fight it"' P7, session 1}

\section{Sub-theme 2: Self-harm as a release}

Self-harm was often used to seek relief from distress, with the hope that the act would release feelings such as anger and frustration. For example, in the extract below, this adolescent describes self-harming to reduce their level of arousal. They explained how they self-harmed to draw blood and this was important for them as the sight and taste of blood had a calming effect:

\footnotetext{
"I think that's maybe why I was self-harming before because I got blood and when I get blood something happens in my mind and I calm down"

P2, session 11
}

In contrast to those who described experiencing relief from self-harm, some individuals wanted to get rid of or stop these feelings. Several participants recalled when feeling angry they harmed themselves to try and stop feeling this way. In both 
scenarios - trying to experience relief from feelings with self-harm and getting rid of feelings using self-harm - the sense of relief is shared. However, trying to seek relief from distressing feelings using self-harm suggests a more temporary solution, whereas using self-harm as a means of trying to rid oneself of feelings implies a more permanent approach. For example, a participant, recalling the aftermath of a disagreement with a parent, wanted some way of managing an overwhelming feeling of rage which engulfed them. They used self-harm to try and rid themselves of their rage, rather than just seeking a reprieve:

"My body just felt like my blood was boiling and I just needed something to get rid of it and I suppose that was it really" P12, session 6

\section{Sub-theme 3: Self-harm to avoid distress}

Both thoughts and acts of self-harm were frequently in response to adolescents remembering distressing events in their lives and ruminating on these memories. Often these memories linked to people in their lives; either loved ones who had passed away or those who had harmed them in some way. Self-harm was used to avoid this distress. For example in the extract below, a participant describes thinking about a particular person who had caused them harm throughout their life and the things that had been done to them. They desperately wanted to avoid these experiences, with the ultimate act of avoidance being portrayed as an attempt to take their own life:

"I'd be sitting in my room thinking about what he's done and it'd be like a hurricane in my head and I would think I'm just going to do it and don't think about whether it hurts or not and when I'm in those bad feelings... and it sounds disgusting and sick but it's the way I feel...I'd happily jump off a bridge and do something and not think about it"

P7, session 1 
As a means of avoiding distress, self-harm was used to replace emotional pain with a more manageable type of suffering; physical pain. For example, a participant described how they hoped that feeling the physical pain would allow them to forget about their sadness. However, they reflected that this strategy did not work - either in the short term or long-term:

A: "To feel pain instead of the sadness"

T: "So you thought that if you hurt yourself the sadness would go away?"

A: "That I would feel the pain and think about that instead of the sadness",

T: "And did that work at all?"

A: "No"

P11, session 1

\section{Sub-theme 4: Self-harm as a form of stimulation}

In contrast to self-harm being used to avoid distress, there were examples of self-harm being utilised to stimulate feelings. For example, a participant described how experiencing a lack of feelings was intolerable, and they used self-harm to feel something. This same individual explained how as well as feeling numb, they did not feel in control of their emotions, and self-harm offered them a way to feel more in control of their emotional world:

\footnotetext{
"It was like when I got really low I felt numb and doing it was a way to feel I guess...I never felt in control of the way I was feeling and it was a way to be in control" P8, session 1
}

Self-harming and feelings of excitement were captured in the response of a participant. They explained how each time they have self-harmed they experienced an adrenaline rush and the act became compulsive: 
"Yeah I think so because erm it's like each time I've selfharmed I've got an adrenaline rush and it feels like something you need to get done"

P5, session 1

\section{Sub-theme 5: Self-harm \& low self-esteem}

A lack of belief and confidence in their own abilities was expressed by some participants. They described feelings of worthlessness, or being worth less than others. In some instances this was related to appearance. For example, a participant explained how they had harmed themselves because they were not satisfied with their body, and cut across their belly in an attempt to lose weight. Another participant expressed a view that they did not have a certain "look". They perceived others to have the look and described feeling both "horrible" and "worthless" because they did not:

"It's horrible...you just feel worthless because you don't

look a certain way... you just feel worthless"

P8, session 6

In response to being reprimanded, another participant described general feelings of worthlessness, not specifically related to their appearance. They then blamed themselves for these feelings which led to them believing that they deserved to be punished, and self-harm was their form of punishment:

"I don't like being told off cos it makes my life feel shitter then what it is and it makes me feel as though I need to punish myself which is why I started self-harming in the first place"

P2, session 4

Low self-esteem was also linked to a perception that those around them, such as friends and family, had rejected them. For example, a participant stated that they had been rejected by their friends and family following negative comments after they had revealed their sexuality. In a later session they started to cry, commenting that all they had wanted was to be "accepted". In the extract below, a participant explains how for 
them, self-harm was an attempt to feel as though they belonged, as they wanted to fit in with their friends who were harming themselves and this influenced their decision to do so:

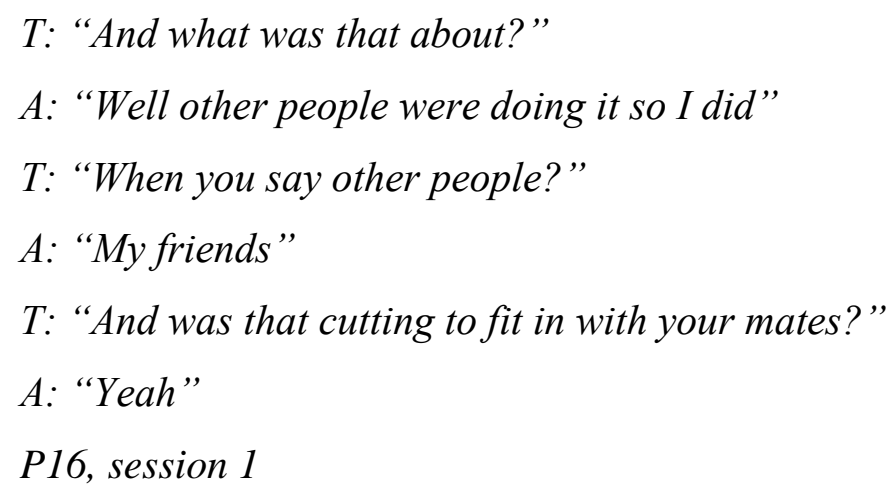

\section{Sub-theme 6: Self-harm \& self-blame}

Evidence of self-blame was present in the accounts of several participants. This was often expressed as being a failure or a belief that they could not do anything right in their lives. They located responsibility for this within themselves. Although one individual did link their feelings of guilt to the emotional abuse they had experienced over many years in the relationship with one of their parents, they stated that they felt guilty for "being born". In this quote, a participant describes how they compared themselves to other people, perceiving others to be happier than they were. They then blamed themselves for this, thinking they had done something wrong - or not done something right - as they were unhappy but others were not:

\footnotetext{
"Well yeah because you see all of these other people who are happy and you think 'what have I done wrong" P22, session 1
}

Similarly, some individuals placed the burden of responsibility on their own shoulders for any problems in their family. In the excerpt below, a participant reflects that they were the cause of, and to blame for, all of the difficulties their family had experienced:

"I was thinking it seems to be me that's the cause of anything that went wrong in the family" P5, session 2 


\section{Theme 5: Moving forward}

This theme, along with the sub-themes, reflects how some participants were able to move forward and resist, or ultimately abstain from, self-harm. Although the process of therapy was usually over the course of a number of months, some participants commenced therapy and were no longer harming themselves, having waited several weeks from referral to their first therapy session. However, for many, the shift from self-harm to ambivalence or resisting the urge to self-harm occurred over the course of therapy. There were a minority of individuals who were able to abstain entirely from self-harm but for the majority the process was characterised as a struggle; on some occasions being successful in resisting the temptation but on other occasions not being able to resist the urge.

\section{Sub-theme 1: Greater self-efficacy}

As described in sub-theme five of theme four, self-harm and low self-esteem, self-harm was linked to low self-esteem and self-worth. The current sub-theme reflects how over the course of therapy some participants developed a belief in their abilities and value as person. For example, in this quote, a participant explains how despite experiencing thoughts of self-harm they had formed new friendships and forged a more positive self-image, which they attributed to helping resist harming themselves:

$$
\begin{aligned}
& \text { T: "And when you get angry and upset is that when you } \\
& \text { start having thoughts that you'll hurt yourself?" } \\
& \text { A: "Yeah but I don't cos I'm cool. I've turned over a } \\
& \text { new leaf and got new friends" } \\
& \text { P4, session } 1
\end{aligned}
$$

Recognising that they had developed alternative strategies for managing distress allowed some participants to resist self-harm. For example, a participant reflected that if they were to resort back to self-harm, this would be back to "square one" and undo all of the hard work they had put in to refrain from harming themselves. In the quote below from a final therapy session, a participant comments that self-harm will always be in their thoughts but they had more confidence in the alternative strategies they had developed, enabling them to resist self-harm: 
T: "And what do you think will happen to your thoughts of wanting to self-harm?"

A: "I think they will always be there because it is part of me but I think now that I have some more ways to cope with it when it's around"

P14, session 11

A belief in their ability to influence their lives and achieve goals was important for some participants in moving forward and resisting self-harm. For example, a participant who had attempted to complete suicide (P22) commented in a later therapy session that they had a more positive focus in their life other than anger, which they reflected had played a part in their attempt to complete suicide. In the quote below, a participant describes how they had enrolled in college and had aspirations for the future:

T: "When was the last time you self-harmed?"

A: "Oh weeks ago"

T: "And what do you make of that?"

A: "Well I've been doing a lot of college work and I guess now I've got goals to aim for and targets"

$P 13$, session 4

\section{Sub-theme 2: Improved emotional regulation}

As highlighted in theme four, the role of self-harm for some was to manage, or control, their emotions. This sub-theme reflects how having more influence over their emotions, or more confidence in their ability to regulate their feelings, enabled some adolescents to resist the urge to harm themselves. For example, in a later therapy session, a participant described their emotions as "more stable" while another rated the likelihood of them taking another overdose as "zero", commenting that they were mentally in a "better place" compared to a previous time when they had attempted to end their life. 
Talking was identified as a healthier way to regulate emotions. For example, in only their second therapy session P18 reflected that their preference was to talk their emotions out rather than punching, which was how they had managed their distress previously (see expressing distress without consequences, theme three). In the quote below, a participant, at the end of one of their later sessions, reflects that they had been able to talk about a very difficult subject, linked to their self-harm, without becoming distressed. They identified this as helpful and recognised this was an on-going process which would take time:

"I suppose the more I talk about and the more I talk about it without tears and get it out the more I can brush it aside...it's still hard though and I think it's going to take years...literally years"

P7, session 2

As well as talking more to regulate emotions, expressing feelings by writing them down was identified as helpful in resisting self-harm by some adolescents. In the quote below, P10 who had resorted to self-harm when angry as they did not know what else to do (see considered vs. uncontrolled self-harm, theme four) describes in this quote how expressing their feelings on paper, and then burning the letters, was helpful some of the time in resisting the urge to harm themselves:

A: "I don't know because I write letters and that and I wrote one to my nan and that even though she's dead and I write my feelings down on paper and I'll take them and burn them on the way to school”

T: "And did that help resist the urge to self-harm?"

A: "Sometimes, it kind of depends how bad I am"

P10, session 2

\section{Sub-theme 3: Being heard, validated \& understood}

In contrast to theme three, self-harm as a form of communication, this sub-theme reflects the way in which being connected socially, and feeling listened to and understood played a role in resisting self-harm. For example, in an earlier therapy 
session, P14 reflected that they had first self-harmed as they wanted their family to stop arguing and recognise how distressed they were (see sub-theme two so others would listen, theme three). In a later session, they went on to explain how having a friend who they felt listened was important and impacted positively on their mood:

\author{
T: "How's your mood been recently?" \\ A: "Well the only noticeable change is when I chat to \\ my friend online because he understands well no \\ he doesn't understand what I'm going through \\ but he's always there to listen to me" \\ P14, session 4
}

As well as being heard, it was important for some adolescents that they had people around them who shared similar experiences and could relate to their own difficulties. For example, a participant reflected on a conversation with a teacher which they found useful as the teacher had admitted that they had experienced difficulties in their own life. This disclosure offered hope to the adolescent, who in the therapy session commented that if the teacher could survive troubled times then so could they. In the following extract, of another adolescent, the parent suggests they could offer comforting words to their child in the same way a friend did. The adolescent's reply indicates that it was not only comforting words that their friend offered that was important; it was also that they had experienced similar difficult life experiences, and could empathise with their situation:

\footnotetext{
"It's not the same though...she has been through abuse as a child and she can relate to it but you can't as you didn't go through that as a kid...she can relate to pretty much anything"
}

\title{
$P 2$, session 6
}

Alongside being listened to and feeling as though others could relate to their difficulties and empathise, it was important for some individuals that those around them offered reassurance. For example, P18 who described experiencing suicidal thoughts due to feeling abandoned (see sub-theme one, clear articulation of intent to 
die, theme two), commented in a later session that it was important to for them to talk their emotions through with individuals, but only those they could count on, and who provided reassurance:

T: "And would you prefer to talk to people rather than fight?"

A: "Yeah I would prefer to talk it out but if I can talk to people I can rely on then I can get all of my emotions out with them and reassurance, that's a big thing, getting reassurance from people"

T: "And who gives you that?"

A: "My family and friends mainly"

P18, session 2

\section{Sub-theme 4: Taking a step back}

In contrast to the sub-theme considered vs. uncontrolled self-harm (theme four) this sub-theme highlights the conversations in which participants were able to step back and reflect on the impact of self-harm. These discussions contributed to a shift in perspective which resulted in some adolescents being able to resist, or cease, selfharm. For example, in the excerpt below, a participant who had attempted to complete suicide explains how they had been able to step-back from their anger and call childline, as they actively wanted to avoid self-harm:

FM: "I think she has called childline a couple of times when she has been upset"

T: "And did you call them when you were upset or for some advice or something different?"

A: "Well one time I did call them because I was really angry and I wanted to know how to deal with it differently"

P17, session 1

Whilst the act of self-harm offered some individuals relief (see sub-theme two of theme four) several commented on the futility of self-harm, liking it to a vicious cycle; 
the act of harming themselves provided benefit in the short-term as it offered a release from their distress but this reprieve was only short-lived. Their distress soon returned and they were then again in a position of wanting to seek relief. For example, in this quote, a participant describes feeling better immediately following an episode of selfharm, but then later feeling even worse than they did prior to the self-harm:

\section{"I don't know I just felt better afterwards but then I felt even worse a few hours later and it just made me do it more and more" \\ P4, session 1}

Reflecting on the distress self-harm caused, such as anxiety family and friends experienced upon learning about the self-harm, facilitated a change in outlook. For example in the quote below, a participant comments that self-harm created a response that had drawn other people in and caused suffering to both them and their parent, and this knowledge motivated them to avoid self-harming again:

“Well yeah in a way, it's like, I don't know I wasn't thinking, I just did it and I wouldn't want to do it again because it doesn't just hurt me it hurts my mum and everything and then everyone gets involved"

\section{P4, session 1}

How others perceived self-harm was important. For example, a participant explained that they were experiencing fewer thoughts of self-harm, which they attributed to having thought about how it causes their parent to worry. They were also concerned with how their parent might be judged by other people as the parent of a child who self-harms. In the excerpt below, another individual describes how resisting self-harm was important to them as it allowed them to feel free from the inaccurate judgements other people make upon those who self-harm:

T: "What's important to you about not self-harming?"

A: "It's important because it frees you from the perception of others and more times than not 
although it maybe true to the person who's thinking

it about you it is completely wrong because only

you know what is going on and their perceptions will

always be wrong"

P14, session 9

Recognising the impact, or potential impact, of friends' self-harm enabled some adolescents to think differently about their own self-harm. For example, in a later therapy session, a participant - who had self-harmed prolifically - described experiencing fewer thoughts of wanting to self-harm having thought over a conversation with a friend had told them that they were planning to self-harm. The participant had reflected to their friend that their family would be upset and that people cared for them. In the extract below from a later therapy session, a participant, who previously stated that they had self-harmed as they "didn't know what else to do" (Considered vs. uncontrolled self-harm, paragraph one theme four), explains how they were motivated to avoid self-harm following a serious incident of a friend harming themselves. They described being shocked and upset, as they had witnessed the fallout and the emotional reaction of the friend's family, as well as realising they had been close to losing a trusted confidant:

\footnotetext{
"I was so upset after it happened and I've seen what it does to other people and I don't want to do that and it's shocked me nearly losing one of my best friends who I tell my problems to" P10, session 5
}

Considering the physical impact of self-harm motivated some individuals to resist harming themselves. For example, a participant reflected that noticing their scars made them think that self-harm was "stupid". In the quote below, a participant describes wanting to resist harming themselves, despite experiencing thoughts to do so, as they did not want to create further scars:

T: "Have there been any other times that you've wanted

to hurt yourself since we last met?" 


\section{A: "Yeah I wanted to but I didn't" \\ T: "And what made you not?" \\ A: "I don't want any more scars" \\ P19, session 2}

\section{Sub-theme 5: Distraction \& substitute behaviours}

Instead of trying to avoid or block out emotions using self-harm, several participants identified distraction techniques as a useful way of managing distress. Strategies included listening to music, watching television, painting, cleaning and talking to people. These tactics were not effective on every occasion when experiencing thoughts of self-harm and described by one participant as only being useful in the short-term. In this quote, a participant explains how when they were feeling down, and thinking about self-harm, they had tried going to sleep or making a hot drink, but actually ended up feeling lower in mood:

"I try to go to sleep or make myself a cup of tea and sometimes it doesn't work and I'll wake up in a foul mood or feel even worse"

P8, session 1

Rather than attempting to distract themselves, other participants substituted self-harm with alternative behaviours. For example, one individual reported scraping their knuckles down a wall when upset. They thought of this as progress, having previously cut and experienced thoughts of wanting to end their life. Another adolescent recalled incidents when they had been extremely angry but had not wanted to self-harm. Instead they had slammed doors, shouted and thrown things. In the quote below, the same participant describes these behaviours as helping to resist the urge to self-harm as they were still able to release their anger. The substitute behaviours were less harmful than the adolescent's self-harming behaviours which included cutting and taking an overdose:

\footnotetext{
"It's like when I get upset I want to do it but I don't as well and when I get upset I go upstairs and starting
} 
banging around and put my music on really loud and that

helps a bit”

P4, session 1

However, it was not always the case that substitute behaviours were less harmful. One adolescent used piercing and smoking as replacements for cutting. They described piercing as a form of self-harm, though they did not consider smoking to be as risky. While piercing may be deemed as more socially acceptable than cutting, and less harmful, smoking is extremely harmful and would be viewed by many as very dangerous:

"Well I've not been self-harming because I've been smoking”

$P 2$, session 2

On the other hand, alternative behaviours described by other adolescents certainly were less harmful, and in some instances actually productive. One individual made music and used rapping as a creative method of releasing their anger, as opposed to punching objects which they had done previously, highlighted in theme three, subtheme three: expressing distress without the consequences:

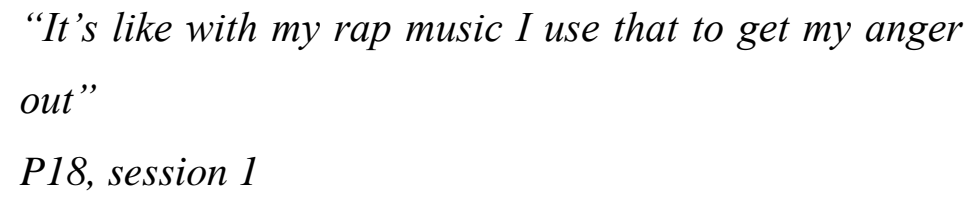

\section{$\underline{\text { Summary }}$}

For some, emotional distress was difficult to discuss. However, some adolescents were able to, and also described a number of reasons which explained why they found it difficult. Accounts highlighted the complex interplay between self-harm and suicidal intent. Whilst self-harm in some cases was linked to an attempt to complete suicide, in other cases it was not about wanting to die. Adding to this complexity were accounts which suggested that self-harm and suicidal intent varied for individuals across, and within, episodes of self-harm. The role of self-harm was diverse. For some it was a means of communicating distress, often in the hope that those around them 
would take action would alleviate distress. Self-harm was also used to manage emotions, although again how self-harm was used to manage emotions varied. Encouragingly, many participants described being able to resist self-harm. How adolescents were able to do this mirrored why some adolescents harmed themselves in the first place. For example, being able to manage their emotions in healthier ways, being more connected and developing a sense of self-efficacy and self-worth contributed to them moving forward. 


\section{CHAPTER FOUR: DISCUSSION}

The results will be summarised and discussed in relation to the existing literature. Strengths and weaknesses of the study will be acknowledged, as well as implications for clinical practice. Areas of future research will be considered, followed by overall conclusions.

\section{$\underline{\text { Summary of findings }}$}

The dataset was gathered via a secondary analysis of video-recorded Family Therapy sessions collected as part of the SHIFT trial. A total of 113 session recordings, from 22 participants, were viewed. Approximately 170 hours of footage formed the dataset. Relevant data was extracted and transcribed. The data were analysed using thematic analysis. Results from the thematic analysis were grouped into five overall themes; (1) Distress can be difficult to convey (2) Self-harm and suicidal ideation; a complex relationship (3) Self-harm as a form of communication (4) Self-harm to manage emotions and (5) Moving forward. The themes and sub-themes will be discussed below in relation to the existing literature.

\section{$\underline{\text { Revisiting research question }}$}

My research question was to understand adolescents' experiences of self-harm, specifically to explore their understandings of why they self-harmed, what their responses to self-harm were, and how they resisted or ceased self-harm.

\section{$\underline{\text { Responses to self-harm }}$}

Adolescents' responses in the therapy sessions varied. Responses related to why they self-harmed, self-harm and suicidal ideation, and how they were able to resist, or cease self-harming, will be discussed further in this chapter. Theme one, Distress can be difficult to convey, highlighted how challenging some of the participants found opening up and discussing their distress. Self-harm, specifically, was a difficult subject to find the words for, which is a common thread within the literature (Hill \& Dallos, 2011; Brown \& Kimball, 2013; Klineberg, Kelly, Stansfield \& Bhui, 2013). When asked questions, some adolescents provided very limited, or no responses. It may have been the case for some that they genuinely did not know the answer, or have the words to label their thoughts and experiences. In contrast to not having the words 
or not wanting to share, there were other adolescents who - though they may have had the words to convey their ideas - stated that they simply did not want to discuss the matter with those present in the therapy room. This may have reflected, more generally, difficulties communicating within the family (Hill \& Dallos, 2011). The consequences of trying to manage alone included bottling their feelings up, resulting in the distress becoming intolerable and thoughts of self-harm or episodes of self-harm. As with previous research (Hill \& Dallos, 2011; Klineberg et al, 2013), fearing, or experiencing, negative responses from others resulted in some adolescents in the present study keeping quiet. Stigma is one of the most frequently identified barriers impacting adolescents' help seeking with research suggesting that adolescents fear disclosing their difficulties due to anticipated social rejection (Gulliver, Griffiths \& Christensen, 2010). Stigma can be thought of as problems in attitude, behaviour and knowledge and is experienced as prejudice, discrimination and ignorance, with adolescents who self-harm at risk of experiencing stigma due to their self-harming behaviour (Mitten, Preyde, Lewis, Vanderkooy \& Heintzman, 2016). Exploring stigmatising attitudes is important as they can impact negatively on an individual's psychological well-being and act as a barrier to treatment seeking, access and adherence (Law, Rostill-Brookes \& Goodman 2009). Adolescents in the current study described some of the responses, related to the wider stigma around self-harm, they had faced from those around them having learned about the self-harming behaviour. In-keeping with previous research, they were viewed as "attention-seeking" (Law, Rostill-Brookes \& Goodman 2009; Klineberg et al, 2013) having self-harmed or they were perceived to be seriously unwell, perhaps even dangerous (Mitten et al, 2016). Given the presence of these stigmatising prominent discourses around self-harm, it is understandable that adolescents report feeling ashamed of self-harming behaviour, and fear being stigmatised (Brown \& Kimball, 2013). This may explain why, as highlighted in this research, some adolescents found it extremely difficult to discuss their difficulties, in particular self-harm.

The idea of self-harm simply being a means of drawing attention to oneself was directly disputed by some adolescents. For example, the suggestion that self-harm was simply for attention was met with one participant stating that self-harm was "not a cry for help" and misunderstood by those around them, which is in keeping with previous research (Hill \& Dallos, 2011; Brown \& Kimball, 2013). In the research by Hill and 
Dallos (2011) six adolescents, 5 females and 1 male aged between 13 and 18 years, were invited to share their life stories. Narrative methodology was used to analyse the data and structure the results. Participants reported feeling misunderstood by others which resulted in them trying to hide their self-harm and actually prevented them from seeking help. Brown and Kimball (2013) used qualitative methodology to explore experiences of self-harm. Participants, 10 female and 1 male, ranging in age from 1939 years were interviewed face-to-face. One of the major categories developed from the results highlighted that individuals thought self-harm was misunderstood and others could not relate to what they were going through. Also, several participants reported feeling isolated from those around them because of self-harm as self-harm created a barrier. Individuals usually want to keep their self-harm private (Hill \& Dallos, 2011; Wadman et al, 2016) and in the current study adolescents pointed out that they had not shown others their self-harm. Wadman et al (2016) interviewed six young adults, aged 19-21 years, about their experiences of self-harm. IPA was used and identified one key theme related to keeping self-harm hidden and secret. In some instances specific parts of the body (for example top of the legs) were chosen to keep self-harm from others. While a consequence of the act of self-harm for participants in the present research may have been that people noticed, the findings - together with previous research - indicate that the intended function of self-harm was more complex. Adolescents wanted others to acknowledge and validate their suffering, and then take action. The idea that others held the view that they were solely "attention seeking" may have actually caused individuals to experience more stress (Mitten et al, 2016) and contributed to them harming themselves as a means of attempting to express their ever increasing distress. These stereotypical, negative responses, then serve to reinforce the idea that self-harm should remain a secret, as the behaviour will be misunderstood by others as an irrational response (Hill \& Dallos, 2011; Brown \& Kimball, 2013). This potentially perpetuates self-harming behaviour as the individual then becomes even less likely to disclose the behaviour to those around them for fear of the reaction (Klineberg et al, 2013; Platt, Kadosh \& Lau, 2013) and contributes to the individual experiencing more distress and then taking action to reduce their level of distress by harming themselves. This may then ultimately feeds the belief that selfharm is not the response of a rational individual. 
The approach, and longitudinal nature, of family therapy afforded adolescents with time and space to reflect on their self-harm. As discussed above, not all were able, or wanted, to do this. Sub-theme four, taking a step back, of theme five, moving forward, in the present research highlights how some participants were able to take a step back and think through self-harm as a response, and the impact of this. These findings compare with those of Wadman et al (2016) who found that participants had started to consider the pros and cons, both emotionally and practically, of self-harm, with some recognising that while self-harm offered relief there was also additional stress associated with the behaviour. In the study by Wadman et al (2016) and in the present research, reflecting on the impact of self-harm contributed to some adolescents reappraising their self-harming behaviour and as consequence being able to resist harming themselves. This was in contrast to those participants who described being unable to consider or control self-harm (described in sub-theme one of theme four, considered vs. uncontrolled self-harm, in the results section of this study). Consistent with previous research exploring self-harm cessation (Gelinas \& Wright, 2013) adolescents in the present study were motivated to resist self-harm as they were worried about the impact their self-harming behaviour may have had on their family and friends, for example not wanting to worry them. They also reflected on the impact of self-harm on themselves. For instance, not wanting any further physical reminders in the form of scars motivated one individual to cease self-harm. This supports the research by Gelinas and Wright (2013). A subset of students -46 females and 8 males ranging in age from 18 to 35 years - who had endorsed engaging in self-harm at one point in their lives, but were no longer harming themselves, were invited to complete an online battery of questionnaires. The results demonstrated that some adolescents were distressed with the physiological impact of self-harm and this playing a part in them ceasing self-harm. The present study extends these findings. As well as considering the impact on themselves, family and friends, participants reflected on the impact and fallout from a friend's self-harm and this contributed in them contemplating the impact of their own self-harm and resulted in a shift, to a position of no longer wanting to harm themselves. 


\section{$\underline{\text { Understanding why adolescents self-harmed }}$}

\section{$\underline{\text { Self-harm to manage emotions }}$}

In keeping with previous research (such as Rodham, Hawton \& Evans, 2004; LloydRichardson, Perrine, Dierker and Kelley, 2007; Brown \& Kimball, 2013; Klineberg, Kelly, Stansfield and Bhui, 2013; Edmondson, Brennan \& House, 2016; Wadman et al, 2016) many individuals in the current study harmed themselves to seek relief from their distress. This was expressed as trying to get rid of painful feelings or using selfharm to achieve a release, often from emotions such as anger or frustration. Adolescents also described harming themselves to take their thoughts away from their distress or to block out painful memories, which replicates findings from earlier research (Young, Van Beinum, Sweeting \& West, 2007; Osuch, Ford, Wrath, Bartha $\&$ Neufeld, 2014). While experiencing difficult emotions was distressing, a lack of feelings was equally as intolerable. Accounts in the current study highlighted selfharm being used to stimulate feelings; to feel something. This this duplicates findings from the research of Lloyd-Richardson, Perrine, Dierker and Kelley (2007) with participants in that research endorsing self-harming to feel, even if the resulting emotion was unpleasant.

Evident in the current study were accounts of using self-harm as a means of replacing emotional pain with physical pain. The physical pain experienced during, or after, self-harm was preferable, or thought of as more manageable, than the emotional distress which had caused participants to seek relief. This supports findings by Brown and Kimball (2013), whose participants described attending to physical pain as being easier than working through emotional pain, but only temporarily, as the distressing feelings would then return. This was reflected in the present study; both in the accounts of adolescents who had self-harmed as a means of replacing emotional pain with physical pain, and in the accounts of adolescents who reflected on the futility of self-harm (sub-theme four, taking a step back, of theme five, moving forward). As in previous research (Brown \& Kimball, 2013; Gelinas and Wright, 2013; Rissanen et al, 2013; Townsend et al, 2016) self-harm, and using physical pain to replace emotional pain, was a useful strategy in the short term, but this was only effective for a brief period of time and then the distress from which the individual had sought relief, returned. Research from the field of coping strategies more broadly distinguishes engagement coping, which is aimed at dealing with the stressor or related emotions, 
and disengagement coping, which is aimed at escaping the threat or related emotions (Carver \& Connor-Smith, 2010). Research by Litman and Lunsford (2009) demonstrated that the overall impact of mental disengagement was marginally positive. However, mental disengagement, including the use of self-harm, predicted illness - as measured in the personal health questionnaire. The authors suggested that even if these strategies (mental avoidance \& self-harm) make people feel better for a short time, there is a longer term cost for suppressing or avoiding unpleasant thoughts and feelings.

Previous research exploring risk factors associated with self-harm has found links between self-harm and low self-esteem (Hawton, Rodham, Evans \& Weatherall, 2002; Portzky, De Wilde \& Van Heeringen, 2008 cited in Fliege et al, 2009). Evidence of low self-esteem was present in the accounts of adolescents in the current study, consistent with prior findings. This was often expressed with statements which highlighted feelings of worthlessness, being inferior to others and rejection. Selfblame and self-derogation, found to be associated with self-harm (Herpetz, Sass \& Favazza, 1997; De Leo \& Heller, 2004 cited in Fliege et al, 2009) was linked to feelings low of self-esteem in the current study. Participants who regarded themselves as a failure located the responsibility for their perceived failings within themselves. This blame was attributed to their own lives, as well as problems experienced in their families, for instance that they were the cause of the difficulties. Self-punishment has been shown to be a reason for self-harm (Rodham, Hawton \& Evans, 2004; LloydRichardson, Perrine, Dierker and Kelley, 2007; Brown \& Kimball, 2013; Edmondson, Brennan \& House, 2016; Wadman et al, 2016). In the current study this selfpunishment was used to manage feelings of low self-esteem and self-blame. Although linked, it is difficult to establish cause and effect for self-blame and low self-esteem. For example, while it is plausible that those individuals who blame themselves for perceived failings then experience lower self-esteem, it is also possible that those who experience lower self-esteem then blame themselves for this. Poor self-esteem is associated with a broad range of mental health difficulties, for example depression, eating disorders and anxiety (Mann, Hosman, Schaalma \& De Vries, 2004). Research exploring a controlled educational intervention targeting self-esteem to improve body image demonstrated improved body image and change in longer term attitudes in male and female adolescents (O'Dea \& Abraham, 2000). This is an encouraging avenue to 
consider for self-harm interventions, for instance targeting self-esteem specifically, and will be discussed further in the clinical implications section of this chapter.

Within the theoretical attempts to understand self-harm, affect regulation has been one of the most prominent reasons put forward as to why individuals harm themselves (Suyemoto, 1998; Klonsky, 2007; Nock, 2010) and this has been supported by primary research (Nock \& Prinstein, 2004; Lloyd-Richardson, Perrine, Dierker and Kelley, 2007; Klineberg, Kelly, Stansfield and Bhui, 2013; Edmondson, Brennan and House, 2016; Wadman et al, 2016). While affect regulation has been widely cited, the current study highlights that this term does not capture the complexity of self-harm as a means of managing emotions and the findings from the present research extend the understanding of this concept. Accounts demonstrated how affect regulation is nuanced, as some participants were trying to master more than one aspect of their emotional world. For example, one participant stated that they had self-harmed because they believed they needed to be punished, yet described another episode when they had harmed themselves to calm down. Responses highlighted how self-harm used to manage emotions may not be captured under the blanket term of affect regulation. Similarly, affect regulation often carries negative connotations, associated with managing distress. While acknowledged in some research (such as Larissa-Bennett \& Moss, 2013; Edmondson, Brennan and House, 2016), self-harm as a positive experience is often overlooked in the literature. Evident in the account of adolescents in the current study were examples of self-harm as a positive action. For example, to feel the rush of adrenaline, which was described as enjoyable. Other accounts portrayed self-harm as a means of protecting, or even benefitting, those around them. Again this contributes to a greater understanding of self-harm to manage emotions and clinical implications of this will be discussed further on.

Control as a major function of self-harm was a finding of the present study, supporting findings from previous research (Hill \& Dallos, 2011; Brown \& Kimball, 2013). Edmondson, Brennan and House (2016) proposed that self-harming to achieve a sense of control may be a positive experience. A participant in the current study reported that when they did not feel in control of their life or choices, self-harm offered control, framed as a positive. Adolescent accounts highlighted that self-harm could either be triggered by an internal (for instance a distressing memory) or external (such as conflict with family) experience. However, rather than a "chicken \& egg" situation - 
for example an episode of self-harm in response to a distressing internal experience triggered by a difficult external experience - perhaps the association is more cyclical, with an individual responding with self-harm at different points in the cycle in an attempt to establish more control over their emotional and external worlds (D Cottrell, personal communication, 2017).

\section{Self-harm as a form of communication}

As highlighted in theme one of the results chapter, distress can be difficult to convey. A number of adolescents found their distress, and self-harm, challenging subjects to discuss, in and out of the therapy sessions. In keeping with previous research (Rodham, Hawton \& Evans, 2004; Hill \& Dallos, 2011) self-harm offered a means by which to highlight to those around them that they were experiencing emotional distress, and the seriousness of this distress, when difficult to put into words. However, the findings in the current study suggest another aspect to self-harm as a form of communication. While they wanted to express the extent of their emotional suffering to friends and family, they also wanted those around them to recognise and acknowledge the level of distress and then take action which would contribute to a change of circumstance and/or a reduction in the adolescent's level of distress. Supporting previous findings (Hill \& Dallos, 2011; Edmondson, Brennan \& House, 2016), accounts were evident in the current study of another group of adolescents who utilised self-harm as a means of expressing their emotional pain, and doing so without facing the consequences which typically accompanies angry behaviour which is directed at others.

\section{$\underline{\text { Self-harm and suicidal ideation; a complex relationship }}$}

Present in the accounts of adolescents was evidence of self-harm explicitly related to suicidal intent, in keeping with previous research (Rodham, Hawton \& Evans, 2004; Klonsky, May \& Glenn, 2013). Self-blame, hopelessness, desperation, worthlessness and suicidal ideation were all linked to attempts to complete suicide (Rutter \& Behrendt, 2004; Klonsky, May \& Glenn, 2013). Suicidal ideation fluctuated and was not always acted on. However, even with intent to end life there was still complexity. For example, a participant who had attempted to end their life by taking an overdose, described in the therapy session how they had experienced both relief - that they had not completed suicide - and disappointment; they had not completed suicide and 
would have to return to school and face the problems which had contributed to them taking the overdose in the first place.

As discussed in the introduction to this research, there is on-going debate in the literature regarding self-harm and suicidal intent, with some suggesting that definitions should explicitly differentiate the concepts (Nock, 2010; Selby, Bender, Gordon, Nock $\&$ Joiner, 2012) and those who propose that intent is difficult to separate out from selfharm (Kapur, Cooper, O’Connor \& Hawton, 2013; Brunner et al, 2014). Findings in the current study demonstrate the complexity of this issue, as accounts of self-harm and suicidal intent co-existed across episodes of self-harm. For instance, some participants reflected that on occasions they had harmed themselves with an intention to complete suicide, yet differentiated episodes of self-harm where there was no intent to die, as found in previous research (Brown \& Kimball, 2013). While the latter would seemingly lend support to a distinct category of NSSI, intent can be difficult to establish, partly as motivations may change from one episode to another and underlying intentions may be unclear even to the person who has harmed themselves (Kapur et al, 2013). This may have been reflected in the current study by those adolescents described in theme one, distress can be difficult to convey, who found it challenging, or were not able, to put into words their motivation for harming themselves.

The findings of the present study do not lend support to the notion of a separate category of self-harm based on intent, such as "non-suicidal self-injury" (Nock, 2010; Selby, Bender, Gordon, Nock \& Joiner, 2012) and concurs with those within the literature who have reservations of NSSI as a distinct classification of self-harm given the challenge of establishing intent (Kapur, Cooper, O'Connor \& Hawton, 2013; Brunner et al, 2014). Taken together with existing literature (Kapur et al, 2013; Edmondson, Brennan \& House, 2016) the findings of the current study indicate that intent is an intricate and challenging concept to establish. The relationship between self-harm and suicidal ideation is nuanced and complex.

\section{$\underline{\text { How adolescents resisted, or ceased, self-harming }}$}

Present in the accounts of some adolescents were descriptions of how they had been able to resist the urge to self-harm or had ceased completely. Often, there seemed to be a shift from the start of therapy, over the course of the sessions - usually a period 
of several months - to a position of ambivalence towards self-harm. As discussed in responses to self-harm earlier in this discussion, taking a step back and reflecting on the impact of self-harm on themselves, those around them and the personal impact of others' self-harm, as well as the futility of self-harm, helped some adolescents to move forward and resist self-harm. The process of resisting self-harm was characterised as difficult, with participants being successful in resisting self-harm on some occasions but not others. A minority of individuals were able to resist the urge each time and stated that they were no longer harming themselves at all.

As highlighted in theme four of the results section, self-harm to manage emotions, one of the functions of self-harm relates to control; offering the individual a means to regulate their emotions and influence their internal and external worlds. In the current study, those individuals who had moved forward, and were able to resist self-harm, described managing their emotions more effectively. These findings support previous research, which has found that individuals who had stopped harming themselves had higher levels of emotional regulation and were able to tolerate stronger emotions (Tatnell, Kelada, Hasking \& Martin, 2014). While it may have been the case in the research by Tatnell et al that emotional regulation increased once self-harm had stopped, accounts from the current study suggest that developing greater emotional regulation coincided with reduced self-harm. Central to improved emotional regulation was stronger connections with those around them and enhanced communication. Vital were better-quality relations with family and friends and being able to share their thoughts and feelings without judgement, or conflict. This increased sense of connection mirrors the findings from the research by Tatnell, Kelada, Hasking and Martin (2014) who found that family support was the most salient factor in cessation of self-harm. Participants were 1973 Australian students aged between 12 and 18 years who completed a questionnaire at two time points, with a twelve-month interval. Those individuals who had ceased harming themselves reported an increase in perceived family support over time. On the other hand lower perceived family support was associated with self-harm. Lower levels of social support and social connectedness have been linked with self-harm (Rotolone \& Martin, 2012). Taken all together; improved emotional regulation, greater connection and improved communication afforded individuals the opportunity to process difficult thoughts and emotions in more constructive ways. 
Participants expressed more confidence in their abilities to select an alternative strategy to manage their distress as therapy progressed. Again, this was in contrast to those individuals who described being unable to consider or control self-harm (described in sub-theme one of theme four of the results section; considered vs. uncontrolled self-harm). Strategies similar to those endorsed by participants in the research by Klonsky and Glenn (2008) were identified and described as helpful; talking to friends and family, distraction and avoiding trigger situations. Talking to friends and family as well as emotional regulation - discussed in the paragraph above - can be thought of as engagement coping strategies (Carver \& Connor-Smith, 2010). As in previous research (Klonsky \& Glenn, 2008) these coping strategies were identified as being effective in resisting the urge to self-harm some, but not all, of the time. Similarly, the alternative behaviours that the adolescents substituted for selfharm were reported as being effective only some of the time. Some of these substitute behaviours were clearly less damaging than the original self-harming behaviour, for example slamming a door instead of cutting. In other instances this was less clear, for instance knuckles scraping down a wall as opposed to cutting. Smoking was one alternative behaviour described by a participant as less damaging than cutting, although many would argue given the health risks associated with smoking that this would not be 'less' harmful (World Health Organisation, 2009). There was one example of an alternative behaviour being productive as the individual made music.

As discussed in theme four of the results chapter of this research, self-harm to manage emotions, self-harm was an attempt to manage feelings of low self-esteem and selfefficacy in some individuals. Research has linked low self-esteem to self-harm (Hawton, Rodham, Evans \& Weatherall, 2002; Rotolone \& Martin, 2012; Tatnell, Kelada, Hasking and Martin, 2014) and higher levels of self-esteem predicted cessation of self-harm in one study (Tatnell, Kelada, Hasking and Martin, 2014). In the current study participants who were able to resist self-harm generally described feeling more positive, and having more belief in their ability to affect change in their lives. These accounts contrasted to those where self-blame and feelings of worthlessness featured heavily. Interestingly, the research by Nielsen and Knardahl (2014) found that disengagement coping strategies, in the form of self-blame, along with self-distraction, were strongly associated with distress at follow-up. A combination of improved emotional regulation, more social connections, a greater 
sense of control and being able to reflect on the impact of self-harm may all have contributed to participants' self-esteem increasing over time in the present study. Developing ambition, hopes for the future and goals to achieve enabled some of the individuals to resist self-harm. This is consistent with research (Weber, 2002) which has identified hope as a powerful construct linked to self-harm cessation.

\section{Summary}

Common themes were identified from the data relating to how adolescents understood their experiences of self-harm. Although several found self-harm a very difficult subject to discuss, many were able to articulate their thoughts and describe why they had harmed themselves. While self-harm was used to manage emotions, this was expressed in a range of ways and a number of reasons for self-harm were evident in the accounts of adolescents. The interplay between self-harm and suicidal ideation was described as complex and difficult to disentangle. On a positive note, adolescents were able to discuss factors important in moving forward and reducing, or ceasing, self-harm.

\section{$\underline{\text { Strengths \& Limitations }}$}

\section{$\underline{\text { Strengths }}$}

\section{Contributions to the literature}

This is the first study to use recorded family therapy sessions to explore adolescents' understanding of their experiences of self-harm. The approach allowed first-hand accounts to be explored, developing an understanding of the experience of self-harm from the perspective of the adolescents.

There have been a number of theoretical attempts, as well as primary studies, aiming to understand why individuals self-harm. As found in previous research, there were a number of reasons why adolescents harmed themselves, and many of the reasons found in the current study replicated previous research. Affect regulation is widely cited as a prominent reason for self-harm. The current research adds to the understanding of self-harm to manage emotions, demonstrating that this is nuanced and complex. There were examples of adolescents using self-harm for more than one purpose in relation to managing feelings. 
This research also found that popular myths surrounding self-harm (for example it is done for "attention") only serve to perpetuate stigma and lead individuals to discuss self-harm less, thus contributing to the secretive nature of self-harm which maintains the problem and ultimately continues to perpetuate the cycle of silence, distress and self-harm.

The findings extend the existing literature on how individuals are able to move forward and resist harming themselves. A greater sense of control, as well as being more connected socially to those around them - such as family and friends - helped develop the adolescents' abilities, and confidence, in resisting self-harm. As well as reflecting on the impact of self-harm on themselves and those around them, evident in the transcripts were examples of adolescents reflecting on the consequences of a friend's self-harm on them. This contributed to a shift in perspective.

There is debate within the literature regarding self-harm and suicidal intent, and whether these two concepts can be thought of as separate or integrated. This research highlighted the complex nature of self-harm and suicidal ideation. It is possible that these two concepts fall on a continuum which fluctuates for the individual - both within and across episodes of self-harm. Therefore, for research purposes it does not make sense to create a distinction between NSSI and suicidal intent.

\section{$\underline{\text { Sample }}$}

The original SHIFT study generated a wealth of data, and I was able to access longitudinal data from 22 participants. This allowed me to fully utilise an existing dataset for appropriate study. As the original trial included both male and females, across the age range of adolescence, with a mix of ethnicities, I was able to utilise a sampling framework to ensure diversity within the participants in this research.

\section{Procedure}

The use of secondary analysis may have captured participants who may have not consented to an interview study about self-harm. As highlighted in the results chapter, self-harm is a sensitive subject for many. It can often be very difficult to describe and find the words to explain the phenomena. With the use of secondary analysis there was no additional burden on the individuals of having to discuss their difficult experiences in more depth. The approach also presented the opportunity of adding a 
richer understanding to the topic of self-harm. For example, rather than selecting reasons from a checklist participants were able to provide detailed accounts.

\section{Quality checks}

As outlined in the method section, Braun and Clarke (2006) offered guidelines that allow thematic analysis to be undertaken more rigorously. I utilised the checklist they provided during the coding, analysis and writing up stages to increase the quality of this research. I also held discussions with my supervisors, experienced researchers, around identifying and refining themes that emerged from the data. I included illustrative quotes verbatim from the participants in support of the themes I constructed.

\section{Limitations}

\section{$\underline{\text { Sample }}$}

As secondary analysis was the methodological approach used in this study, the sample was limited by the choices made by the researchers' in the SHIFT trial. For example, adolescents under care of the local authority and living in a children's home, or in short-term foster care, were not eligible. This excludes a subset of the population that may be more at risk of harming themselves as research has found that adolescents in the Looked after Care (LAC) system demonstrate high rates of self-harm (James, Winmill, Anderson \& Alfoadari, 2011).

Similarly, participants in the SHIFT trial were selected on the basis that their selfharming behaviour resulted in an admission to Hospital/Accident and Emergency, or they were already under the care of primary services. Less lethal methods of self-harm and intention to die have been associated with receiving help from services (Klineberg, Kelly, Stansfield \& Bhui, 2013). Rodham, Hawton and Evans (2004) found that only $12.6 \%$ of their sample, of 361 students who had reported self-harming, had gone to Hospital as a result of their self-harm. Therefore, those adolescents that were selfharming but did not seek help, or did not receive treatment as a result of their selfharm, would not have been captured by the SHIFT trial. It is plausible to consider that those adolescents seeking help were more open to conversations relating to self-harm. For example, those adolescents in the family therapy treatment arm did, albeit possibly reluctantly, attend the therapy session and communicated in some way, shape or form. 
It may be that if data were collected from a sample of adolescents who were harming themselves, but not seeking help, they would have offered alternative accounts which would not have reflected the themes generated in this study. Likewise, I only had access to those participants who had consented to take part in the SHIFT trial and agreed to have their sessions recorded and be available for use in future research. It may be those individuals were contemplating their self-harming behaviour already and more willing to change.

\section{Procedure}

The use of secondary analysis, as opposed to the use of a primary data collection method, meant that as a researcher I was somewhat constrained by the dataset. For example, the use of a primary data collection method (such as semi-structured interview or focus group) would have allowed me the opportunity to explore a participant's response in more detail. I would have then been able to ask follow up questions, clarify a response and even pursue a new line of enquiry if relevant. On a number of occasions I found myself hoping the SHIFT trial therapists continued with a certain line of questioning which was relevant to my research aims.

The process of forming the dataset was incredibly time consuming - approximately 170 hours of footage was viewed. As the sole researcher viewing the sessions it is plausible to consider that I missed data relevant to the research question due to fatigue. I often watched multiple session recordings in one day. It is possible that I was not attending to parts of a session which would have offered data that contradicts the results I generated, or provided an alternative perspective to the analysis presented.

\section{$\underline{\text { Researcher bias }}$}

It is important to point out that researcher bias can never be eliminated (Braun \& Clark, 2006) although I had taken steps to reduce this. For example, I reflected on my position approaching the data, I detailed the data collection and analysis process, used a journal to record my initial responses to session recordings, included illustrative quotes to back up my analytic claims and had supervision with an experienced qualitative researcher. However, my role as a Trainee Clinical Psychologist, along with my own life experiences, may have influenced the data I selected and transcribed for later analysis. For instance, in the role as a trainee I occupy both the role of researcher and clinician at various points. While in the role of researcher I may wear 
a different 'hat' (approaching the role from a different perspective) though it is likely that my experiences when wearing my clinical 'hat' influence me when wearing my research 'hat', and vice versa.

\section{$\underline{\text { Retrospective accounts }}$}

It should be acknowledged that during the family therapy sessions the adolescents were often, although not always, reflecting on episodes of self-harm that had occurred sometime previously. The information provided during the sessions was the adolescent's construct of reality at that time, described in a way to make sense of their experiences. This does not guarantee accuracy, as we only have partial awareness, and not complete access, to our experiences. The translation of reflective awareness into languaged expression might not have fully captured their experience of self-harm, particularly if offered sometime after the experience actually occurred (Polkinghorne, 2005).

It is possible to consider that the adolescents, when reflecting on their experiences of self-harm with a therapist and/or their family in a therapy session, may have made sense of their experience of self-harm in a different way if they had been interviewed without the presence of others. For example, they will have only talked about things they were willing to allow their parents and family to know. Therefore the presence of family members may have influenced the data collection and subsequent analysis.

\section{$\underline{\text { Clinical implications }}$}

There are a number of reasons why understanding self-harming behaviour among adolescents is important. Research suggests the self-harm is on the rise (Rowe, French, Henderson, Ougrin, Slade \& Moran, 2014) and, crucially, adolescents who self-harm are at higher risk of a repeated episode and self-harm is a key risk factor in completed suicide (Klonsky, May \& Glenn, 2013). While there have been numerous theoretical and primary studies aimed at understanding self-harm, this study, in line with recommendations from previous research (Brown and Kimball, 2013) has explored first-hand accounts of those adolescents who self-harm to better understand their unique experiences of self-harm. Continuing to further our understanding of selfharm, both theoretically and via primary research methods, adds to and furthers the 
existing literature which then hopefully informs social awareness and ultimately treatment.

This study, along with previous findings, highlights how distress, including self-harm, can be difficult to discuss. However, it is crucial that adolescents are presented with opportunities to communicate their distress. Research has found how there is little open communication and considerable scope for stigma and fear around self-harm as parents feel scared of discussing the issue, teachers feel unsure of what they can do and many GPs were concerned that they did have the language to talk about self-harm. All of which results in young people not knowing where to turn for help (Cello \& Young Minds, 2012). Providing safe, non-judgemental spaces is crucial in facilitating difficult discussions with adolescents. Literature from therapy outcomes demonstrates that the therapeutic alliance is considered a key common factor in therapy, making substantial and consistent contributions to positive outcomes (Norcross \& Wampold, 2011). Martin, Garske and Davis (2000) suggest that most theoretical definitions of the therapeutic alliance have three common themes; (i) the collaborative nature of the relationship (ii) the affective bond between service user and therapist and (iii) the service user and therapist's ability to agree on treatment goals and tasks. As well as therapy, these common themes apply generally to those who engage with adolescents (for instance parents, teachers, GPs) and are important in the process of creating safe spaces to facilitate discussions. Conversations then provide adolescents with the opportunity to reflect on, and make sense of, their experiences of self-harm. While some will no doubt find this extremely difficult, this does not mean conversations should not take place or they should not be offered the opportunity. Even if they were to decline, as some in this study did, this implies that self-harm is acceptable to discuss and may reduce shame and self-stigma (Cello \& Young Minds, 2012). In the current study, data recorded as part of family therapy sessions was utilised. However, this is only one approach. Participants in the present study identified numerous alternatives, such as talking with friends or family, writing thoughts and feelings down, and communicating online to name a few. As highlighted in theme five, moving forward, in the results section, being able to reflect on experiences of self-harm contributed to a shift in perspective which ultimately played a key role in some adolescents being able to resist self-harm, or even cease completely. 
The current study, along with previous research (Hill \& Dallos, 2011; Brown \& Kimball, 2013; Klineberg, Kelly, Stansfield \& Bhui, 2013; Edmondson, Brennan \& House, 2016; Wadman et al, 2016) highlights that self-harm has a function in the life of the individual. While affect regulation is widely endorsed as a reason for self-harm, the findings of this research demonstrate the complexity of self-harm to manage emotions. Concentrating solely on affect regulation and developing strategies to manage emotions more effectively becomes ultimately less useful unless the idea of regulating emotions using self-harm is explored in depth with the individual. There appears to be benefit in approaches aimed at helping adolescents develop a greater sense of control in their lives given the important role gaining control appears to play in self-harm (Hill \& Dallos, 2011; Brown \& Kimball, 2013). With regards to therapy there is scope to offer adolescents control with respect to the day and time when they are seen, where they are seen (for example home or community) or therapeutic modality. While possibly viewed as arbitrary, taken together they can contribute to the adolescent feeling more in control of, and engaging more in, an intervention.

Given the literature which highlights the link between lower levels of connectedness and lower levels of social support and self-harm, any intervention would benefit from trying to increase connectedness in the lives of adolescents. One way in which to go about this would be discussions identifying what is important to the individual and helping them move towards behaviour which is in line with their values (Hayes, Luoma, Bond, Masuda \& Lillis, 2006). Similarly, the present study, combined with existing research (Mumme, Mildred and Knight, 2016; Palmer, Walsh and Tiffin, 2016) demonstrates the importance of incorporating family support in an intervention aimed at reducing self-harm. Including parents, siblings and extended family into any intervention - where possible and appropriate - appears to be beneficial. This will require careful consideration and need to be in collaboration with the adolescent.

O'Dea and Abraham (2000) successfully targeted self-esteem as an intervention to improve body image and to change longer term attitudes in male and female adolescents. The current study, along with previous research, has linked low selfesteem to self-harm (Hawton, Rodham, Evans \& Weatherall, 2002; Rotolone \& Martin, 2012; Tatnell, Kelada, Hasking and Martin, 2014). Given that the adolescent may be using self-harm to manage distress, an intervention solely aimed at reducing self-harm may - from the adolescent's perspective - be attempting to remove their 
only strategy to manage distress, or even a positive experience for them. Therefore, developing an adolescent's longer-term engagement coping strategies, such as seeking support and talking, alongside a specific self-esteem based intervention may offer a way forward in clinical interventions. This would allow the individual to develop skills which would help longer term and not just in the short term (Litman \& Lunsford, 2009) whilst boosting their self-worth.

The current study demonstrates the complex relationship between self-harm and suicidal ideation, with self-harm not always being related to suicidal intent (Brown \& Kimball, 2013). This is an important consideration for individuals working with adolescents to bear in mind as over half of GPs, teachers and parents think that young people who self-harm are likely to try and commit suicide (Cello \& Young Minds, 2012). While suicidal intent should be explored and the risk assessed, it should not be assumed. Equally, it is vital to revisit intent throughout an intervention as adolescents' motivations can change over time and across episodes of self-harm.

\section{$\underline{\text { Research implications }}$}

Future work could compare data collected, using primary data collection methods such as interview, with the themes produced in the current study. An interview would allow the researcher the opportunity to explore a participant's response in more detail. During the data collection phase there were a number of points in therapy sessions that the SHIFT trial therapists could have pursued a participant's answer to elicit more understanding, potentially opening up even more conversations. However, the approach of Family Therapy focuses more on circular questioning and the sessions were therapy, not research. Therefore, research involving primary data collection methods would allow the interviewer to ask follow-up questions to clarify a response. They would also be able to pursue new lines of enquiry and potentially develop a richer understanding. Similarly, the themes generated in the current research could be explored using quantitative methodology. For instance, the findings have highlighted how self-harm to manage emotions is nuanced and complex. This could be reflected in future questionnaires, with a number of choices to select under a heading of 'affect regulation' or 'self-harm to manage emotions'. This could then be combined with open questions in order to capture more detail and build a richer understanding of an individual's self-harm to manage emotions. 
It would be interesting to investigate the themes in a longitudinal study, particularly with understudied populations. As described in the method section, participants in the SHIFT trial were selected on this basis that their self-harming behaviour resulted in in an admission to Hospital/Accident and Emergency, or they were already under the care of primary services. Although possibly harder to reach, there are a number of adolescents harming themselves who are not seeking help. Therefore, it would be beneficial to compare how the themes produced in the current study compared to data gathered from a sample that may potentially be approaching self-harm from a different perspective, such as not even contemplating managing in a different way. Increasing diversity within the sample of any future research exploring first-hand accounts of selfharm in adolescents would be beneficial. For example, those adolescents who are in the care of the local authority and in the looked after system (LAC). Research has shown that this subset of the population may be more at risk of harming themselves (James, Winmill, Anderson \& Alfoadari, 2011). If a future sample included data collected from either, or both, of these subsets of the population, then alternative accounts, not reflecting the themes produced in this study, may be generated.

There is debate within the literature as to whether definitions of self-harm should differentiate intent (for example suicidal vs. non-suicidal, Nock, 2010) and whether intent can be reliably reported (Kapur, Cooper, O'Connor \& Hawton, 2013; Brunner et al 2014). This has led to a number of definitions being used in the literature to define the behaviour (such as "self-mutilative behaviour", Nock and Prinstein, 2004; “deliberate self-injury” Klonsky 2007, \& “Non-suicidal self-injury” Nock \& Favazza, 2009, cited in Zetterqvist, Lundh, Dahlstrom \& Svedin, 2013) all of which can be confusing for individuals searching for relevant information relating to the behaviour (for instance adolescents/parents/professionals) and a barrier to research (Brunner et al, 2014). As highlighted in the current study, there were examples of self-harm being explicitly related to suicidal intent, examples of self-harm explicitly not linked with suicidal intent and instances where intent was less clear. Taking all of this into account, it seems important that one definition (such as self-harm) encompasses all of these which can then be explored with an individual to understand their intent for each episode of self-harm.

As discussed, the current study focussed on how adolescents understood their experiences of self-harm. Another potentially valuable avenue for future research 
could explore the role of dialogue between family members in relation to self-harm. This offers the opportunity to differentiate how parents, adolescents and potentially siblings, talk about self-harm. One way to do this would be to replicate the current study and utilise the existing data set collected as part of the SHIFT trial. It would then be interesting to explore how data collected in such research compared to the themes developed in the current study. Particularly in relation to family members' views on the role of self-harm and how these fit with prominent discourses around self-harm.

\section{$\underline{\text { Conclusions }}$}

- Emotional distress, and specifically self-harm, can be difficult subjects for adolescents to discuss. While this was challenging for the majority, many were able to articulate their thoughts and make sense of their experiences.

- Prominent social discourses around self-harm and the myths perpetuated resulted in some adolescents attempting to manage alone and fearing the consequences should they open up and talk about self-harm.

- Self-harm is "not a cry for help" or "attention seeking" by adolescents. It is a means of expressing the seriousness of emotional distress to those around them.

- There are a number of reasons why adolescents may self-harm although selfharm is often an attempt to exert more control over one's internal and external worlds. Self-harm is frequently used as a strategy to regulate emotions. However, how emotions are regulated varies.

- While self-harm and suicide ideation was sometimes linked, the association was complex. There were clear instances of self-harm with the intent to end life, episodes with no intent to end life and self-harm where intent was less clear. Motivation also changed across episodes.

- $\quad$ Those around them taking action, where possible, to contribute to a reduction in distress is helpful although acknowledging and validating distress is key.

- A process which encourages reflection and "taking a step back" can contribute to adolescents reflecting on their self-harming behaviour and facilitating a change in outlook. 
- Developing stronger social connections, with improved communication, can help those adolescents who are self-harming to reduce, or cease, harming themselves. 


\section{REFERENCES}

Anderson, C. (2010) Presenting and evaluating qualitative research. American Journal of Pharmaceutical Education, 74 (8).

Akyuz, G., Sar, V., Kugu, N., \& Doğan, O. (2005) Reported childhood trauma, attempted suicide and self-mutilative behaviour among women in the general population. European Psychiatry, 20(3), 268-273. Cited in Fliege, H., Lee, J. R., Grimm, A., \& Klapp, B. F. (2009). Risk factors and correlates of deliberate self-harm behaviour: A systematic review. Journal of psychosomatic research, 66(6), 477-493.

Baker, S.E., \& Edwards, R. (2012) How many qualitative interviews is enough? Expert voices and early career reflections in sampling and cases in qualitative research. Retrieved from: http://eprints.brighton.ac.uk/id/eprint/11632, November $30^{\text {th }} 2015$.

Bjorkenstam, C., Kosidou, K., \& Bjorkenstam, E. (2017) Childhood adversity and risk of suicide: cohort study of 548721 adolescents and young adults in Sweden. BMJ, 357, 13-34.

Braun, V., \& Clarke, V. (2006) Using thematic analysis in psychology. Qualitative Research in Psychology, 3 (2), 77-101.

Braun, V., \& Clarke, V., (2013) Successful qualitative research: A practical guide for beginners. London: Sage publications.

Briere, J., \& Gil, E. (1998) Self-mutilation in clinical and general population samples: Prevalence, correlates, and functions. American Journal of orthopsychiatry, 68(4), 609-620. Cited in Fliege, H., Lee, J. R., Grimm, A., \& Klapp, B. F. (2009). Risk factors and correlates of deliberate self-harm behaviour: A systematic review. Journal of psychosomatic research, 66(6), 477493.

Brunner, R., Kaess, M., Parzer, P., Fischer, G., Carli, V., Hoven, C.W., Wasserman, C., Sarchiapone, M., Resch, F., Apter, A., Balazs, J., Barzilay, S., Bobes, J., Corcoran, P., Cosmanm, D., Haring, C., Iosuec, M., Kahn, J.P., Keeley, H., Meszaros, G., Nemes, B., Podlogar, T., Postuvan, V., Saiz, P.A., Sisask, M., Tubiana, A., Varnik, A., \& Wasserman, D. (2014) Life-time prevalence and psychosocial correlates of adolescent direct self-injurious behaviour: A comparative study of 11 European countries. Journal of Child Psychology and Psychiatry, 55 (4), 337-348.

Brown, T.B., \& Kimball, T. (2013) Cutting to live: A phenomenology of self-harm. Journal of Marital and Family Therapy, 39 (2), 195-208.

Burr, V (2015) Social Constructionism, $3^{\text {rd }}$ edition. London, New York: Routledge, Taylor \& Francis group.

Carver, C.S., \& Connor-Smith, J. (2010) Personality and coping. Annual Review of Psychology, 61, 679-704.

Cello \& Minds, Y. (2012) Talking self-harm. 
De Leo, D., \& Heller, T. S. (2004). Who are the kids who self-harm? An Australian self-report school survey. Medical journal of Australia, 181(3), 140-144. Cited in; Fliege, H., Lee, J. R., Grimm, A., \& Klapp, B. F. (2009). Risk factors and correlates of deliberate self-harm behaviour: A systematic review. Journal of psychosomatic research, 66(6), 477-493.

Dixon-Woods, M. (2011) Using framework-based synthesis for conducting reviews of qualitative studies. BMC Medicine, 9 (1).

Edmondson, A.J., Brennan, C.A., \& House, A.O. (2016) Non-suicidal reasons for self-harm: A systematic review of self-reported accounts. Journal of Affective Disorders, 191, 109-117.

Favazza, A.R. (1998) The coming age of self-mutilation. The Journal of nervous and mental disease, 186 (5), 259-268.

Fliege, H., Lee, J. R., Grimm, A., \& Klapp, B. F. (2009). Risk factors and correlates of deliberate self-harm behaviour: A systematic review. Journal of psychosomatic research, 66(6), 477-493.

Gee, J.P. (2014) An introduction to Discourse Analysis: theory and method, $4^{\text {th }}$ edition. London, New York: Routledge, Taylor \& Francis group.

Gelinas, B.L., \& Wright, K.D. (2013) The cessation of deliberate self-harm in a University sample: the reasons, barriers and strategies involved. Archives of Suicide Research, 17 (4), 373-386.

Graneheim, U.H., \& Lundman, B (2004) Qualitative content analysis in nursing research: concepts, procedures, and measures to achieve trustworthiness. Nurse Education Today, 24, 105-112.

Gratz, K. L., Conrad, S. D., \& Roemer, L. (2002) Risk factors for deliberate selfharm among college students. American Journal of Orthopsychiatry, 72(1), 128. Cited in Fliege, H., Lee, J. R., Grimm, A., \& Klapp, B. F. (2009). Risk factors and correlates of deliberate self-harm behaviour: A systematic review. Journal of psychosomatic research, 66(6), 477-493.

Guest, G., Bunce, A., \& Johnson, L. (2006) How many interviews are enough? An experiment with data saturation and variability. Field Methods, 18, 59-82.

Gulliver, A., Griffiths, K.M., \& Christensen, H. (2010) Perceived barriers and facilitators in mental health help-seeking in young people: a systematic review. BMC Psychiatry, 10 (1), 113.

Gutierrez, P.M., Osman, A., Barrios, F.X., \& Klopper, B.A. (2001) Development and initial validation of the self-harm behaviour questionnaire. Journal of Personality Assessment, 77 (3), 475-490.

Haley, T. (2002) The fit between reflecting teams and a social constructionist approach. Journal of Systemic Therapies, 21 (1), pp 20-40.

Hawton, K., Rodham, K., Evans, E., \& Weatherall, R. (2002) Deliberate self-harm in adolescents: self-report survey in schools in England. British Medical Journal, $325,1207-1211$. 
Hawton, K., \& Rodham, K., \& Evans, E. (2006) By their own young hand: deliberate self-harm and suicidal ideas in adolescence. London \& Philadelphia: Jessica Kingsley.

Hawton, K., Saunders, K.E., \& O'Connor, R.C. (2012) Self-harm and suicide in adolescents. The Lancet, 379, (9834), 2372-2382.

Hayes, S.C., Luoma, J.B., Bond, F.W., Masuda, A., \& Lillis, J. (2006) Acceptance and Commitment Therapy: Model processes and outcomes. Behaviour Research and Therapy, 44, 1-25.

Heaton, J. (2003) Secondary analysis of qualitative data. In R.L. Miller \& J.D. Brewer (Eds) The A-Z of Social Research (280-284). London: Sage publications.

Herpertz, S., Sass, H., \& Favazza, A. (1997) Impulsivity in self-mutilative behaviour: psychometric and biological findings. Journal of psychiatric research, 31(4), 451- 465. Cited in; Fliege, H., Lee, J. R., Grimm, A., \& Klapp, B. F. (2009). Risk factors and correlates of deliberate self-harm behaviour: A systematic review. Journal of psychosomatic research, 66(6), 477-493.

Hill, K., \& Dallos, R. (2011) Young people's stories of self-harm: A narrative study. Clinical Child Psychiatry and Psychiatry, 17 (3), 459-475.

Hinds, P. S., Vogel, R. J., \& Clarke-Steffen, L. (1997) The possibilities and pitfalls of doing a secondary analysis of a qualitative data set. Qualitative Health Research, 7(3), 408-424.

James, A. C., Winmill, L., Anderson, C., \& Alfoadari, K. (2011) A preliminary study of an extension of a community dialectic behaviour therapy (DBT) programme to adolescents in the looked after care system. Child and Adolescent Mental Health, 16 (1), 9-13.

Jenkins, D. (1996) A reflecting team approach to family therapy: A delphi study. Journal of Marital and Family Therapy, 22, 219-238. Cited in; Haley, T. (2002). The fit between reflecting teams and a social constructionist approach. Journal of Systemic Therapies, 21 (1), pp 20-40.

Kapur, N., Cooper, J., O’Connor, R., \& Hawton, K. (2013) Non-suicidal self-injury v. attempted suicide: new diagnosis or false dichotomy. The British Journal of Psychiatry, 202, 326-328.

Kirkcaldy, B. D., Brown, J., \& Siefen, R. G. (2006) Disruptive behavioural disorders, self-harm and suicidal ideation among German adolescents in psychiatric care. International journal of adolescent medicine and health, 18(4), 597-614. Cited in Fliege, H., Lee, J. R., Grimm, A., \& Klapp, B. F. (2009). Risk factors and correlates of deliberate self-harm behaviour: A systematic review. Journal of psychosomatic research, 66(6), 477-493.

Klineberg, E., Kelly, M.J., Stansfield, S.A., \& Bhui, K.S. (2013) How do adolescents talk about self-harm: a qualitative study of disclosure in an ethnically diverse urban population in England. BMC Public Health, 13 (1), 572.

Klonsky, D.E. (2007) The functions of deliberate self-injury: A review of the evidence. Clinical Psychology Review, 27, 226-239. 
Klonsky, D.E. (2011) Non-suicidal self-injury in United States adults: Prevalence, socio-demographics, topography and functions. Psychological Medicine, 41 (9), 1981-1986.

Klonsky, D.E., Oltmanns, T. F., \& Turkheimer, E. (2003) Deliberate self-harm in a nonclinical population: Prevalence and psychological correlates. American journal of Psychiatry, 160(8), 1501-1508.

Klonsky, D.E., \& Glenn, C.R. (2008) Resisting urges to self-harm. Behavioural and Cognitive Psychotherapy, 36, 211-220.

Klonsky, D.E., May, A.M., \& Glenn, C.R. (2013) The relationship between nonsuicidal self-injury and attempted suicide: converging evidence from four samples. Journal of Abnormal Psychology, 122 (1), 231-237.

Larissa Bennett, A., \& Moss, M. (2013) Functions of deliberate self-injury of personality disordered prisoners: a small scale study. The Journal of Forensic Practice, 15(3), 171-181.

Law, G.U., Rostill-Brookes, H., \& Goodman, D. (2009) Public stigma in health and non-healthcare students: Attributions, emotions and willingness to help with adolescent self-harm. International journal of nursing studies, 46(1), 108-119.

Laye-Gindhu, A., \& Schonert-Reichl, K.A. (2005) Non-suicidal self-harm among community adolescents: Understanding the "whats" and "whys" of self-harm. Journal of Youth and Adolescence, 34 (5), 447-457.

Lincoln, Y.S., \& Guba, E. (1985) Naturalistic Enquiry. Sage, Thousand Oaks, CA. Cited in Tobin, G.A., \& Begley, C.M. (2004) Methodological rigour within a qualitative framework. Journal of Advanced Nursing, 48 (4), 388-396. Cited in Tobin, G.A., \& Begley, C.M. (2004) Methodological rigour within a qualitative framework. Journal of Advanced Nursing, 48 (4), 388-396.

Litman, J.A., \& Lunsford, G.D. (2009) Frequency of use and impact of coping strategies assessed by the COPE Inventory and their relationships to post-event health and well-being. Journal of Health Psychology, 14(7), 982-991.

Lloyd, E.E., Kelley, M.L., \& Hope, T. (1997) Self-mutilation in a community sample of adolescents: Descriptive characteristics and provisional prevalence rates. Poster session presented at the annual meeting of the Society for Behavioural Medicine, New Orleans, LA. Cited in Nock, M.K., \& Prinstein, M.J. (2004) A functional approach to the assessment of self-mutilative behaviour. Journal of Consulting and Clinical Psychology, 72 (5), 885-890.

Lloyd-Richardson, E.E., Perrine, N., Dierker, L., \& Kelley, M. (2007) Characteristics and functions of non-suicidal self-injury in a community sample of adolescents. Psychological Medicine, 37, 1183-1192.

Madge, N., Hewitt, A., Hawton, K., Jan de Wilde, E., Corcoran, P., Fekete, S., van Heeringen, K., De Leo, D., \& Ystgaard, M. (2008) Deliberate self-harm within an international community sample of young people: comparative findings from the Child \& Adolescent Self-harm in Europe (CASE) study. Journal of Child Psychology and Psychiatry, 49 (6), 667-677. 
Mann, M. M., Hosman, C. M., Schaalma, H. P., \& De Vries, N. K. (2004) Selfesteem in a broad-spectrum approach for mental health promotion. Health education research, 19(4), 357-372.

Mitten, N., Preyde, M., Lewis, S., Vanderkooy, J., \& Heintzman, J. (2016) The perceptions of adolescents who self-harm on stigma and care following inpatient psychiatric treatment. Social Work in Mental Health, 14(1), 1-21.

Moran, P., Coffey, C., Romaniuk, H., Olsson, C., Borschmann, R., Carlin, J. B., \& Patton, G. C. (2012) The natural history of self-harm from adolescence to young adulthood: a population-based cohort study. The Lancet, 379 (9812), 236-243.

Muehlenkamp, J.J., Claes, L., Havertape, L., \& Plener, P.L. (2012) International prevalence of adolescent non-suicidal self-injury and deliberate self-harm. Child and Adolescent Psychiatry and Mental Health, 6 (10), 1-9.

Mumme, T.A., Mildred, H., \& Knight, T. (2016) How do people stop non-suicidal self-injury? A systematic review. Archives of suicide research, 1-20.

NICE (2011) Self-harm in over 8s: long term management. Retrieved from http://www.nice.org.uk/CG133.

Nielsen, M.B., \& Knardahl, S. (2014) Coping strategies: A prospective study of patterns, stability, and relationships with psychological distress. Scandinavian Journal of Psychology, 55(2), 142-150.

Nock, M.K. (2010) Self-injury. Annual review of Clinical Psychology, 6 (1), 15.115.25 .

Nock, M.K., \& Prinstein, M.J. (2004) A functional approach to the assessment of self-mutilative behaviour. Journal of Consulting and Clinical Psychology, 72 (5), 885-890.

Nock, M.K., \& Favazza, A.R. (2009) Non-suicidal self-injury: Definition and classification. In M.K. Nock (ed) Understanding self-injury. Origins, assessment and treatment (pp 9-18). Washington, DC: American Psychological Association. Cited in Zetterqvist, M., Lundh, L.G., Dahlstrom, O., \& Svedin, C.G. (2013) Prevalence and function of non-suicidal self-injury (NSSI) in a community sample of adolescents, using suggested DSM-5 criteria for a potential NSSI disorder. Journal of Abnormal Child Psychology, 41, 759-773.

O'Dea, J. A., \& Abraham, S. (2000) Improving the body image, eating attitudes, and behaviours of young male and female adolescents: A new educational approach that focuses on self-esteem. International Journal of Eating Disorders, 28(1), 43- 57.

Osuch, E., Ford, K., Wrath, A., Bartha, R., Neufeld, R. (2014) Functional MRI of pain application in youth who engaged in repetitive non-suicidal self-injury vs psychiatric controls. Psychiatric Research, 223 (2), 104-112.

Owens, D., Kelley, R., Munyombwe, T., Bergen, H., Hawton, K., Cooper, J., Ness, J., Waters, K., West, R., \& Kapur, N. (2015) Switching methods of self-harm at repeat episodes: findings from a multicentre cohort study. Journal of Affective Disorders, 180, 44-51.

Palmer, E., Welsh, P., \& Tiffin, P.A. (2016) Perceptions of family functioning in adolescents who self-harm. Journal of Family Therapy, 38, 257-273. 
Platt, B., Kadosh, K.C., and Lau, J.Y. (2013) The role of peer rejection in adolescent depression. Depression and Anxiety, 30 (9), 809-821.

Polit, D.F., \& Hungler, B.P. (1999) Nursing Research: Principles and Methods, sixth ed. Philadelphia, New York, Baltimore: J.B. Lippincott Company. Cited in Graneheim, U.H., \& Lundman, B. (2004) Qualitative content analysis in nursing research: concepts, procedures, and measures to achieve trustworthiness. Nurse Education Today, 24, 105-112.

Polkinghorne, D.E. (2005) Language and meaning: Data collection in qualitative research. Journal of Counseling Psychology, 52 (2), 137-145.

Portzky, G., De Wilde, E. J., \& Van Heeringen, K. (2008) Deliberate self-harm in young people: differences in prevalence and risk factors between The Netherlands and Belgium. European child \& adolescent psychiatry, 17(3), 179186. Cited in Fliege, H., Lee, J. R., Grimm, A., \& Klapp, B. F. (2009). Risk factors and correlates of deliberate self-harm behaviour: A systematic review. Journal of psychosomatic research, 66(6), 477-493.

Rissanen, M.L., Kylma, J., Hintikka, J., Honkalampi, K., Tolmunen, T., \& Laukkanen, E. (2013) Factors helping adolescents to stop self-cutting: descriptions of 347 adolescents aged 13-18 years. Journal of Clinical Nursing, 22, 2011-2019.

Ritchie, J., \& Lewis, J. (2003) Qualitative Research Practice: a guide for Social Science Students and Researchers. London: Sage publications.

Robinson, J. (2016) Repeated self-harm in young people: a review. Australasian Psychiatry. DOI: 1039856216679542

Rodham, K., Hawton, K., \& Evans, E. (2004) Reasons for deliberate self-harm: comparison of self-poisoners and self-cutters in a community sample of adolescents. Journal of American Academic Child and Adolescent Psychiatry, 43 (1), 80-87.

Rotolone, C., \& Martin, G. (2012) Giving up self-injury: a comparison of everyday social and personal resources in past versus current self-injurers. Archives of Suicide Research, 16(2), 147-158.

Rowe, S.L., French, R.S., Henderson, C., Ougrin, D., Slade, M., \& Moran, P. (2014) Help-seeking behaviour and adolescent self-harm: A systematic review. Australian \& New Zealand Journal of Psychiatry, 48 (12), 1083-1095.

Rutter, P. A., \& Behrendt, A. E. (2004) Adolescent suicide risk: Four psychosocial factors. Adolescence, 39(154), 295.

Schmidtke, A., Bille-Brahe, U., DeLeo, D., Kerkhof, A. F. J. M., Bjerke, T., Crepef, P., \& Pommereau, X. (1996) Attempted suicide in Europe: rates, trends and sociodemographic characteristics of suicide attempters during the period 19891992. Results of the WHO/EURO Multicentre Study on parasuicide. Acta Psychiatrica Scandinavica, 93(5), 327-338. Cited in Fliege, H., Lee, J. R., Grimm, A., \& Klapp, B. F. (2009). Risk factors and correlates of deliberate selfharm behaviour: A systematic review. Journal of psychosomatic research, 66(6), 477-493. 
Selby, E. A., Bender, T. W., Gordon, K. H., Nock, M. K., \& Joiner Jr, T. E. (2012) Non-suicidal self-injury (NSSI) disorder: a preliminary study. Personality Disorders: Theory, Research, and Treatment, 3(2), 167.

Smith, J.A. (2015) Qualitative Psychology: A practical guide to research methods. London: Sage publications.

Stratton, P.M., Munton, A.G., Hanks, H.G.I., Heard D.H., \& Davison, C. (1988) Leeds Attributional Coding System (LACS) Manual. Leeds: LFTRC. Cited in Stratton, P.M., \& Bromley, K. (1999) Families' accounts of the causal processes in food choices. Appetite, 33, 89-108.

Stratton, P.M., \& Bromley, K. (1999) Families' accounts of the causal processes in food choices. Appetite, 33, 89-108.

Suyemoto, K. L. (1998) The functions of self-mutilation. Clinical Psychology Review, 18 (5), 531-554.

Swannell, S.V., Martin, G.E., Page, A., Hasking, P., \& St John, N.J. (2014) Prevalence of Non-suicidal Self-injury in Nonclinical Samples: Systematic Review, Meta-analysis and Meta-regression. Suicide and life-threatening behaviour, 44 (3), 273-303.

Tatnell, R., Kelada, L., Hasking, P., \& Martin, G. (2014) Longitudinal analysis of adolescent NSSI: the role of intrapersonal and interpersonal factors. Journal of Child Psychology, 42, 885-896.

Tiffin, P.A. (2007) Development and validation of the family perceptions scale. MA thesis. Newcastle University. Cited in; Palmer, E., Welsh, P., \& Tiffin, P.A. (2016) Perceptions of family functioning in adolescents who self-harm. Journal of Family Therapy, 38, 257-273.

Tobin, G.A., \& Begley, C.M. (2004) Methodological rigour within a qualitative framework. Journal of Advanced Nursing, 48 (4), 388-396.

Townsend, E., Wadman, R., Sayal, K., Armstrong, M., Harroe, C., Majumber, P., Vostanis, P., \& Clarke, D. (2016) Uncovering key patterns in self-harm in adolescents: Sequence analysis using the card sort task for self-harm (CaTS). Journal of affective disorder, 206, 161-168.

Van der Kolk, B. A., Perry, J. C., \& Herman, J. L. (1991) Childhood origins of selfdestructive behaviour. The American journal of psychiatry, 148(12), 1665. Cited in Cited in Fliege, H., Lee, J. R., Grimm, A., \& Klapp, B. F. (2009). Risk factors and correlates of deliberate self-harm behaviour: A systematic review. Journal of psychosomatic research, 66(6), 477-493.

Victor, S.E., \& Klonsky, E.D. (2014) Correlates of suicide attempts among selfinjurers: A meta-analysis. Clinical Psychology Review, 34 (4), 282-297.

Wadman, R., Clarke, D., Sayal, K., Vostanis, P., Armstrong, M., Harroe, C., Majumber, P., \& Townsend, E. (2016) An interpretative phenomenological analysis of the experience of self-harm repetition and recovery in young adults. Journal of Health Psychology, 1-11.

Weber, M.T. (2002) Triggers for self-abuse: a qualitative study. Archives of Psychiatric Nursing, 16 (3), 118-124. 
World Health Organization. (2009) Global health risks: mortality and burden of disease attributable to selected major risks. World Health Organization.

Wright, J., Friedrich, W., Cinq-Mars, C., Cyr, M., \& McDuff, P. (2004) Selfdestructive and delinquent behaviours of adolescent female victims of child sexual abuse: rates and covariates in clinical and nonclinical samples. Violence and Victims, 19(6), 627-643. Cited in Cited in Cited in Fliege, H., Lee, J. R., Grimm, A., \& Klapp, B. F. (2009). Risk factors and correlates of deliberate selfharm behaviour: A systematic review. Journal of psychosomatic research, 66(6), 477-493.

Wright-Hughes, A., Graham, E., Farrin, A., Collinson, M., Boston, P., Eisler, I., Fortune, S., Green, J., House, A., Owens, D., Simic, M., Tubeuf, S., Nixon, J., McCabe, C. Kerfoot, M., \& Cottrell, D. (2015) Self-harm intervention: Family Therapy (SHIFT), a study protocol for a randomised controlled trial of family therapy versus treatment as usual for young people seen after a second or subsequent episode of self-harm. Trials, 16:501, 1-12.

Young, R., Van Beinum, M., Sweeting, H., West, P. (2007) Young people who selfharm. British Journal of Journal of Psychiatry, 191 (1), 44-49.

Zetterqvist, M., Lundh, L.G., Dahlstrom, O., \& Svedin, C.G. (2013) Prevalence and function of non-suicidal self-injury (NSSI) in a community sample of adolescents, using suggested DSM-5 criteria for a potential NSSI disorder. Journal of Abnormal Child Psychology, 41, 759-773. 


\title{
APPENDICES
}

Appendix 1: Ethics approval granted by the NHS

\section{WHS \\ Health Research Authority}

\author{
London - South East Research Ethics Committee \\ Barlow House \\ 3rd Floor \\ 4 Minshull Street \\ Manchester \\ M1 3DZ
}

03 March 2016

Mr Robert Holliday

Psychologist in Clinical Training

Leeds Teaching Hospitals NHS Trust

Clinical Psychology training programme

Leeds Institute of Health Sciences, Charles Thackrah Building

101 Clarendon Road, Leeds

LS2 9LU

Dear Mr Holliday

Study title:

How do adolescents talk about self-harm: a secondary analysis of SHIFT trial data

REC reference:

16/LO/0362

IRAS project ID:

199026

Thank you for your submission, responding to the Proportionate Review Sub-

Committee's request for changes to the documentation for the above study.

The revised documentation has been reviewed and approved by the sub-committee.

We plan to publish your research summary wording for the above study on the HRA website, together with your contact details. 
Publication will be no earlier than three months from the date of this favourable opinion letter. The expectation is that this information will be published for all studies that receive an ethical opinion but should you wish to provide a substitute contact point, wish to make a request to defer, or require further information, please contact the REC Manager, Margaret Hutchinson, nrescommittee.londonsoutheast@nhs.net. Under very limited circumstances (e.g. for student research which has received an unfavourable opinion), it may be possible to grant an exemption to the publication of the study.

Confirmation of ethical opinion

On behalf of the Committee, I am pleased to confirm a favourable ethical opinion for the above research on the basis described in the application form, protocol and supporting documentation as revised.

Conditions of the favourable opinion

The REC favourable opinion is subject to the following conditions being met prior to the start of the study.

Management permission must be obtained from each host organisation prior to the start of the study at the site concerned.

Management permission should be sought from all NHS organisations involved in the study in accordance with NHS research governance arrangements. Each NHS organisation must confirm through the signing of agreements and/or other documents that it has given permission for the research to proceed (except where explicitly specified otherwise).

Guidance on applying for HRA Approval (England)/ NHS permission for research is available in the Integrated Research Application System, www.hra.nhs.uk or at http://www.rdforum.nhs.uk.

Where a NHS organisation's role in the study is limited to identifying and referring potential participants to research sites ("participant identification centre"), guidance should be sought from the $R \& D$ office on the information it requires to give permission for this activity.

For non-NHS sites, site management permission should be obtained in accordance with the procedures of the relevant host organisation. 
Sponsors are not required to notify the Committee of management permissions from host organisations.

Registration of Clinical Trials

All clinical trials (defined as the first four categories on the IRAS filter page) must be registered on a publically accessible database. This should be before the first participant is recruited but no later than 6 weeks after recruitment of the first participant.

There is no requirement to separately notify the REC but you should do so at the earliest opportunity e.g. when submitting an amendment. We will audit the registration details as part of the annual progress reporting process.

To ensure transparency in research, we strongly recommend that all research is registered but for non-clinical trials this is not currently mandatory.

If a sponsor wishes to request a deferral for study registration within the required timeframe, they should contact hra.studyregistration@nhs.net. The expectation is that all clinical trials will be registered, however, in exceptional circumstances non registration may be permissible with prior agreement from the HRA. Guidance on where to register is provided on the HRA website.

It is the responsibility of the sponsor to ensure that all the conditions are complied with before the start of the study or its initiation at a particular site (as applicable).

Ethical review of research sites

The favourable opinion applies to all NHS sites taking part in the study, subject to management permission being obtained from the NHS/HSC R\&D office prior to the start of the study (see

"Conditions of the favourable opinion" above).

Approved documents

The documents reviewed and approved by the Committee are:

\begin{tabular}{|l|l|l|}
\hline Document & Version & Date \\
\hline $\begin{array}{l}\text { Evidence of Sponsor insurance or indemnity (non NHS Sponsors } \\
\text { only) [University Indemnity] }\end{array}$ & 1 & 22 September 2015 \\
\hline IRAS Checklist XML [Checklist_15022016] & & 15 February 2016 \\
\hline
\end{tabular}




\begin{tabular}{|l|l|l|}
\hline REC Application Form [REC_Form_15022016] & & 15 February 2016 \\
\hline Research protocol or project proposal [Protocol] & 1 & 15 February 2016 \\
\hline Summary CV for Chief Investigator (CI) [RH CV] & & 03 March 2016 \\
\hline Summary CV for supervisor (student research) [Supervisor CV] & 1 & 15 February 2016 \\
\hline
\end{tabular}

\section{Statement of compliance}

The Committee is constituted in accordance with the Governance Arrangements for Research Ethics Committees and complies fully with the Standard Operating Procedures for Research Ethics Committees in the UK.

After ethical review

Reporting requirements

The attached document "After ethical review - guidance for researchers" gives detailed guidance on reporting requirements for studies with a favourable opinion, including:

- Notifying substantial amendments

- Adding new sites and investigators

- Notification of serious breaches of the protocol

- Progress and safety reports

- Notifying the end of the study

The HRA website also provides guidance on these topics, which is updated in the light of changes in reporting requirements or procedures.

Feedback

You are invited to give your view of the service that you have received from the National Research Ethics Service and the application procedure. If you wish to make your views known please use the feedback form available on the HRA website: http://www.hra.nhs.uk/about-thehra/governance/quality-assurance

We are pleased to welcome researchers and R \& D staff at our NRES committee members' training days - see details at http://www.hra.nhs.uk/hra-training/ 
With the Committee's best wishes for the success of this project.

Yours sincerely

PP K. Southoe

On behalf of Professor David Caplin Chair

Email: nrescommittee.london-southeast@nhs.net

Enclosures: $\quad$ After ethical review - guidance for researchers

Copy to: $\quad$ Faculty Research Ethics \& Governance Administrator Ms Anne Gowing, Leeds Teaching Hospitals NHS trust $\square$ 
Appendix 2: R\&D approval

$\begin{array}{lr}\text { Leeds Community Healthcare WHS } \\ \text { NHS Trust } \\ \text { for you, for life } & \\ \text { Telephone enquiries, please contact: } & \text { Stockdale House } \\ \text { Phone: } \quad 0113 \text { 8433497 / 07710148229 } & \text { Headingley Office Park } \\ \text { Email: } \quad \text { Ich.research@nhs.net } & \text { Victoria Road } \\ 22^{\text {nd }} \text { March } 2016 & \text { Leeds LS6 1PF }\end{array}$

Robert Holliday

Psychologist in Clinical Training

Leeds Teaching Hospitals NHS Trust

Clinical Psychology training programme

Leeds Institute of Health Sciences, Charles Thackrah Building

101 Clarendon Road, Leeds

LS2 9LU

IRAS Ref: 199026

REC Ref: 16/LO/0362

LCH Ref: SE/044

Dear Mr Holliday

Re: How do adolescents talk about self-harm: a secondary analysis of SHIFT trial data

Thank you for your recent request to Leeds Community Healthcare (LCH) NHS Trust requesting NHS Research Governance approval for the above study.

Following consideration of your submission I can confirm that your study does not require a local research governance review, however we are issuing this letter to you as acknowledgment of your project.

The R\&D team wish you every success with your study.

Yours sincerely

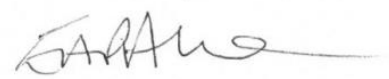

\section{Dr. Liz Allen}

Research Manager

Approved documents

The documents reviewed and approved are listed below

\begin{tabular}{|l|l|l|}
\hline Document & Version & Date of Document \\
\hline REC approval & & 03.03 .2016 \\
\hline Protocol & 1 & 15.02 .2016 \\
\hline Sponsor identified & Univeristy of Leeds & \\
\hline
\end{tabular}




\section{Appendix 3: Examples of NVIVO analysis}

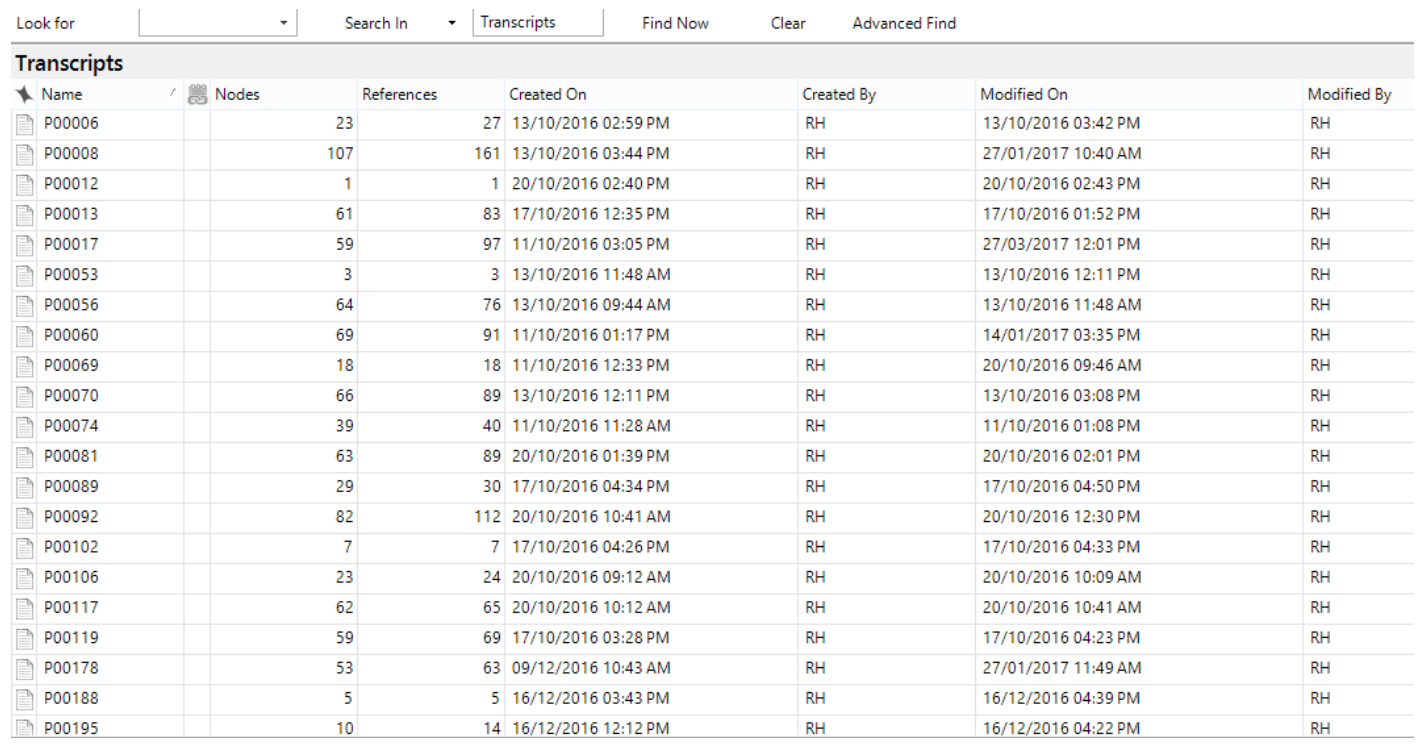

\begin{tabular}{|c|c|c|c|c|c|c|}
\hline \multicolumn{7}{|l|}{ Nodes } \\
\hline Name & Sources & Referenc & Created By & Created On & Modified By & Modified On \\
\hline (T. Version 3 & 0 & 0 & RH & 05/01/2017 04:01 PM & $\mathrm{RH}$ & 05/01/2017 04:01 PM \\
\hline+0 Version 2 & 0 & 0 & RH & 05/01/2017 04:05 PM & $\mathrm{RH}$ & 05/01/2017 04:05 PM \\
\hline (t) Version 5 & 0 & 0 & $\mathrm{RH}$ & 27/01/2017 10:24 AM & $\mathrm{RH}$ & 19/03/2017 02:35 PM \\
\hline$\forall$ Version 7 & 0 & 0 & RH & 21/04/2017 10:33 AM & RH & 21/04/2017 10:33 AM \\
\hline Setting the scene & 0 & 0 & RH & 21/04/2017 10:33 AM & RH & 15/11/2016 04:04 PM \\
\hline$\because$ Abuse & 0 & 0 & $\mathrm{RH}$ & 21/04/2017 10:33 AM & $\mathrm{RH}$ & 05/01/2017 04:13 PM \\
\hline Sexual abuse & 1 & 1 & $\mathrm{RH}$ & 21/04/2017 10:33 AM & $\mathrm{RH}$ & $27 / 01 / 2017$ 10:36 AM \\
\hline Emotional abuse & 2 & 2 & $\mathrm{RH}$ & 21/04/2017 10:33 AM & $\mathrm{RH}$ & $27 / 01 / 2017$ 10:36 AM \\
\hline Physical abuse & 2 & 2 & RH & 21/04/2017 10:33 AM & RH & 27/01/2017 11:02 AM \\
\hline Bullying & 3 & 3 & RH & 21/04/2017 10:33 AM & RH & 27/03/2017 02:55 PM \\
\hline Subtance use & 3 & 4 & RH & 21/04/2017 10:33 AM & RH & 27/01/2017 10:36 AM \\
\hline Self-harm as a form of communication & 0 & 0 & RH & 21/04/2017 10:33 AM & RH & 24/04/2017 02:16 PM \\
\hline Abandoned & 1 & 1 & RH & 21/04/2017 10:33 AM & RH & 27/03/2017 02:24 PM \\
\hline Belonging & 1 & 1 & RH & 21/04/2017 10:33 AM & RH & 02/12/2016 11:38 AM \\
\hline Being heard & 2 & 2 & RH & 21/04/2017 10:33 AM & $\mathrm{RH}$ & 18/03/2017 04:42 PM \\
\hline Not a cry for help & 3 & 3 & $\mathrm{RH}$ & 21/04/2017 10:33 AM & $\mathrm{RH}$ & 28/03/2017 09:52 AM \\
\hline Respone to conflict & 4 & 6 & $\mathrm{RH}$ & 21/04/2017 10:33 AM & $\mathrm{RH}$ & 21/04/2017 05:05 PM \\
\hline Expressing distress without the consequences & 5 & 5 & RH & 21/04/2017 10:33 AM & $\mathrm{RH}$ & 28/04/2017 01:21 PM \\
\hline
\end{tabular}




\begin{tabular}{|c|c|c|c|c|c|c|}
\hline Nodes & & & & & & \\
\hline Name & Sources & Referenc & c cy Created By & Created On & Modified By & Modified On \\
\hline Self harm \& suicidal ideation, a complex relationship & & 0 & $\mathrm{RH}$ & 21/04/2017 10:33 AM & $\mathrm{RH}$ & 03/02/2017 09:09 AM \\
\hline$\oplus \bigcirc$ Clear articulation of intent to die & & 0 & RH & 21/04/2017 01:29 PM & $\mathrm{RH}$ & 21/04/2017 01:29 PM \\
\hline Ambivalence towards suicide & & 1 & $\mathrm{RH}$ & 21/04/2017 10:33 AM & $\mathrm{RH}$ & 15/01/2017 03:10 PM \\
\hline Desperation & & 1 & RH & 21/04/2017 10:33 AM & RH & 02/12/2016 02:08 PM \\
\hline Sick of life & & 1 & $\mathrm{RH}$ & 21/04/2017 10:33 AM & $\mathrm{RH}$ & 15/02/2017 02:53 PM \\
\hline Worthlessness & & 1 & $\mathrm{RH}$ & 25/04/2017 10:49 AM & $\mathrm{RH}$ & 25/04/2017 10:49 AM \\
\hline Persecuted & & 1 & $\mathrm{RH}$ & 25/04/2017 10:55 AM & $\mathrm{RH}$ & 25/04/2017 10:55 AM \\
\hline Thoughts fluctuate & & 2 & $\mathrm{RH}$ & 25/04/2017 10:04 AM & $\mathrm{RH}$ & 27/03/2017 12:16 PM \\
\hline Intent but not acting on it & & 2 & $\mathrm{RH}$ & 25/04/2017 10:05 AM & $\mathrm{RH}$ & 25/04/2017 10:06 AM \\
\hline Self-blame & & 2 & $\mathrm{RH}$ & 25/04/2017 10:48 AM & $\mathrm{RH}$ & 25/04/2017 10:59 AM \\
\hline Suicidal ideation & & 4 & $\mathrm{RH}$ & 21/04/2017 10:33 AM & $\mathrm{RH}$ & 27/03/2017 11:06 AM \\
\hline Hopelessness & & 4 & RH & 21/04/2017 10:33 AM & $\mathrm{RH}$ & 27/03/2017 12:14 PM \\
\hline Not wanting to die & & 0 & $\mathrm{RH}$ & 21/04/2017 01:33 PM & $\mathrm{RH}$ & 21/04/2017 01:33 PM \\
\hline Not an attempt to complete suicide & & 1 & $\mathrm{RH}$ & 21/04/2017 10:33 AM & $\mathrm{RH}$ & 28/04/2017 11:46 AM \\
\hline Trying to stay alive & & 1 & $\mathrm{RH}$ & 21/04/2017 10:33 AM & $\mathrm{RH}$ & 15/01/2017 02:00 PM \\
\hline Escape problems & & 2 & RH & 21/04/2017 10:33 AM & RH & 27/03/2017 10:59 AM \\
\hline Thoughts fluctuate & & 2 & $\mathrm{RH}$ & 21/04/2017 10:33 AM & $\mathrm{RH}$ & 27/03/2017 12:16 PM \\
\hline E. Self-harm \& suicide co-existing & & 3 & $\mathrm{RH}$ & 21/04/2017 10:33 AM & $\mathrm{RH}$ & 21/04/2017 01:31 PM \\
\hline
\end{tabular}

\begin{tabular}{|c|c|c|c|c|c|c|c|}
\hline \multicolumn{8}{|l|}{ Nodes } \\
\hline Name & Sources & & Referenc & Created By & Created On & Modified By & Modified On \\
\hline O scarification & & 1 & 1 & $\mathrm{RH}$ & 21/04/2017 10:33 AM & $\mathrm{RH}$ & 25/04/2017 02:31 PM \\
\hline Moving forward & & 0 & 0 & $\mathrm{RH}$ & 21/04/2017 10:33 AM & $\mathrm{RH}$ & 03/02/2017 01:46 PM \\
\hline Taking a step back & & 0 & 0 & $\mathrm{RH}$ & 21/04/2017 10:33 AM & $\mathrm{RH}$ & 25/04/2017 02:30 PM \\
\hline Waste of resources & & 1 & 1 & $\mathrm{RH}$ & 21/04/2017 10:33 AM & $\mathrm{RH}$ & 25/04/2017 03:26 PM \\
\hline Reframing & & 1 & 1 & $\mathrm{RH}$ & 21/04/2017 10:33 AM & $\mathrm{RH}$ & 14/01/2017 06:20 PM \\
\hline Guilt & & 1 & 1 & $\mathrm{RH}$ & 21/04/2017 10:33 AM & RH & 27/03/2017 12:01 PM \\
\hline Wanting to manage in a different way & & 1 & 1 & $\mathrm{RH}$ & 25/04/2017 03:24 PM & $\mathrm{RH}$ & 25/04/2017 03:24 PM \\
\hline Impact of other's self-harm & & 2 & 2 & $\mathrm{RH}$ & 21/04/2017 10:33 AM & $\mathrm{RH}$ & 10/01/2017 02:26 PM \\
\hline Consequences for others & & 2 & 3 & $\mathrm{RH}$ & 21/04/2017 10:33 AM & $\mathrm{RH}$ & 27/03/2017 11:29 AM \\
\hline Perception of others & & 2 & 2 & $\mathrm{RH}$ & 21/04/2017 10:33 AM & $\mathrm{RH}$ & 25/03/2017 03:39 PM \\
\hline Ambivalence towards self-harm & & 4 & 6 & $\mathrm{RH}$ & 21/04/2017 10:33 AM & $\mathrm{RH}$ & 25/03/2017 02:08 PM \\
\hline Futility of self-harm & & 4 & 4 & RH & 01/05/2017 02:15 PM & $\mathrm{RH}$ & 01/05/2017 02:16 PM \\
\hline Distraction \& substitute behaviours & & 0 & 0 & $\mathrm{RH}$ & 21/04/2017 10:33 AM & $\mathrm{RH}$ & 03/02/2017 02:51 PM \\
\hline Substitute behaviours & & 5 & 13 & $\mathrm{RH}$ & 21/04/2017 10:33 AM & $\mathrm{RH}$ & 27/03/2017 04:05 PM \\
\hline Distraction & & 6 & 9 & $\mathrm{RH}$ & 21/04/2017 10:33 AM & $\mathrm{RH}$ & 27/03/2017 11:20 AM \\
\hline Being heard, validated \& understood & & 0 & 0 & $\mathrm{RH}$ & 21/04/2017 10:33 AM & $\mathrm{RH}$ & 25/04/2017 04:53 PM \\
\hline More assertive & & 1 & 1 & $\mathrm{RH}$ & 21/04/2017 10:33 AM & $\mathrm{RH}$ & 27/03/2017 03:24 PM \\
\hline Less conflict & & 2 & 3 & $\mathrm{RH}$ & 21/04/2017 10:33 AM & $\mathrm{RH}$ & 14/02/2017 03:05 PM \\
\hline$\cap$ Reassured & & 2 & 2 & $\mathrm{RH}$ & $21 / 04 / 201710: 33$ AM & $\mathrm{RH}$ & 27/03/2017 04:01 PM \\
\hline
\end{tabular}

\begin{tabular}{|c|c|c|c|c|c|c|}
\hline \multicolumn{7}{|l|}{ Nodes } \\
\hline Name & Sources & Referenc & Created By & Created On & Modified By & Modified On \\
\hline Supportive network & 4 & 4 & $\mathrm{RH}$ & 21/04/2017 10:33 AM & $\mathrm{RH}$ & 28/03/2017 09:53 AM \\
\hline Being heard & 8 & 12 & $\mathrm{RH}$ & 21/04/2017 10:33 AM & $\mathrm{RH}$ & 28/03/2017 10:01 AM \\
\hline$=$ Greater self-efficacy & 0 & 0 & $\mathrm{RH}$ & 21/04/2017 10:33 AM & $\mathrm{RH}$ & 25/04/2017 03:32 PM \\
\hline Back to square one & 1 & 1 & $\mathrm{RH}$ & 21/04/2017 10:33 AM & $\mathrm{RH}$ & 14/01/2017 05:10 PM \\
\hline Hopes for the future & 1 & 1 & RH & 21/04/2017 10:33 AM & $\mathrm{RH}$ & 05/12/2016 03:46 PM \\
\hline Different focus & 1 & 1 & $\mathrm{RH}$ & 21/04/2017 10:33 AM & $\mathrm{RH}$ & 28/03/2017 11:04 AM \\
\hline Physical reminders & 2 & 2 & $\mathrm{RH}$ & 21/04/2017 10:33 AM & $\mathrm{RH}$ & 27/03/2017 03:00 PM \\
\hline Alternative strategies & 2 & 2 & $\mathrm{RH}$ & 21/04/2017 10:33 AM & $\mathrm{RH}$ & 28/03/2017 10:16 AM \\
\hline Positive self image & 2 & 2 & $\mathrm{RH}$ & 21/04/2017 10:33 AM & $\mathrm{RH}$ & 25/04/2017 03:33 PM \\
\hline Avoid triggers & 3 & 4 & $\mathrm{RH}$ & 21/04/2017 10:33 AM & $\mathrm{RH}$ & 19/03/2017 01:14 PM \\
\hline$\because$ Improved emotional regulation & 0 & 0 & $\mathrm{RH}$ & 25/04/2017 03:33 PM & $\mathrm{RH}$ & 25/04/2017 03:33 PM \\
\hline Writing to process emotions & 2 & 2 & $\mathrm{RH}$ & 25/04/2017 03:57 PM & $\mathrm{RH}$ & 25/04/2017 03:59 PM \\
\hline Talking to process emotions & 3 & 3 & $\mathrm{RH}$ & 21/04/2017 10:33 AM & $\mathrm{RH}$ & 25/04/2017 04:00 PM \\
\hline More stable & 3 & 3 & RH & 25/04/2017 03:56 PM & $\mathrm{RH}$ & 25/04/2017 03:57 PM \\
\hline A battle & 4 & 5 & $\mathrm{RH}$ & 21/04/2017 10:33 AM & $\mathrm{RH}$ & 27/01/2017 10:33 AM \\
\hline Distress can be difficult to convey & 0 & 0 & $\mathrm{RH}$ & 21/04/2017 10:33 AM & $\mathrm{RH}$ & 01/05/2017 10:44 AM \\
\hline Trying to manage alone & 0 & 0 & $\mathrm{RH}$ & 21/04/2017 01:27 PM & $\mathrm{RH}$ & 21/04/2017 01:27 PM \\
\hline Creates stress & 1 & 2 & $\mathrm{RH}$ & 21/04/2017 10:33 AM & $\mathrm{RH}$ & 09/12/2016 03:20 PM \\
\hline
\end{tabular}




\begin{tabular}{|c|c|c|c|c|c|c|}
\hline \multicolumn{7}{|l|}{ Nodes } \\
\hline Name & Sources & Referenc & Created By & Created On & Modified By & Modified On \\
\hline Self-harm to manage emotions & & 0 & $\mathrm{RH}$ & 22/04/2017 11:32 AM & $\mathrm{RH}$ & 22/04/2017 11:32 AM \\
\hline Considered vs, uncontrolled self-harm & & 0 & $\mathrm{RH}$ & 21/04/2017 10:33 AM & $\mathrm{RH}$ & $21 / 04 / 201704: 29 \mathrm{PM}$ \\
\hline 由. Inevitable & & 1 & RH & 21/04/2017 10:33 AM & $\mathrm{RH}$ & 27/01/2017 10:47 AM \\
\hline Urge is unpredictable & & 1 & $\mathrm{RH}$ & 21/04/2017 10:33 AM & $\mathrm{RH}$ & 08/01/2017 03:56 PM \\
\hline Limited options & & 1 & $\mathrm{RH}$ & 21/04/2017 10:33 AM & $\mathrm{RH}$ & $13 / 01 / 2017$ 01:30 PM \\
\hline Compulsive & & 2 & $\mathrm{RH}$ & 21/04/2017 10:33 AM & RH & $27 / 01 / 2017$ 10:47 AM \\
\hline Impulsive & & 2 & $\mathrm{RH}$ & 21/04/2017 10:33 AM & $\mathrm{RH}$ & 28/03/2017 09:55 AM \\
\hline Powerless & & 2 & $\mathrm{RH}$ & 21/04/2017 10:33 AM & RH & 27/01/2017 10:34 AM \\
\hline Self-harm to avoid, block out feelings & & 0 & RH & 24/04/2017 02:21 PM & $\mathrm{RH}$ & 24/04/2017 02:21 PM \\
\hline Replacing emotional pain with physical pain ( & & 2 & $\mathrm{RH}$ & 21/04/2017 10:33 AM & RH & $22 / 11 / 201611: 06 \mathrm{AM}$ \\
\hline Painful memories & & 9 & $\mathrm{RH}$ & 21/04/2017 10:33 AM & $\mathrm{RH}$ & 28/03/2017 10:56 AM \\
\hline 10 Self-harm as a form of self-punishment & & 0 & $\mathrm{RH}$ & 24/04/2017 02:22 PM & $\mathrm{RH}$ & 24/04/2017 02:22 PM \\
\hline Self-punishment & & 2 & RH & 21/04/2017 10:33 AM & $\mathrm{RH}$ & 27/01/2017 10:36 AM \\
\hline Guilt & & 2 & $\mathrm{RH}$ & 21/04/2017 10:33 AM & RH & 27/03/2017 02:29 PM \\
\hline Worthlessness & & 3 & $\mathrm{RH}$ & 21/04/2017 10:33 AM & $\mathrm{RH}$ & 27/01/2017 10:33 AM \\
\hline Self blame & & 8 & $\mathrm{RH}$ & 21/04/2017 10:33 AM & $\mathrm{RH}$ & 21/03/2017 11:09 AM \\
\hline Self-harm as a form of stimulation & & 0 & $\mathrm{RH}$ & 24/04/2017 02:25 PM & $\mathrm{RH}$ & 24/04/2017 02:25 PM \\
\hline Numb, need to feel & & 1 & $\mathrm{RH}$ & 21/04/2017 10:33 AM & $\mathrm{RH}$ & 14/01/2017 03:12 PM \\
\hline
\end{tabular}

\begin{tabular}{|c|c|c|c|c|c|c|}
\hline \multicolumn{7}{|l|}{ Nodes } \\
\hline Name & Sources & Referenc & Created By & Created On & Modified By & Modified On \\
\hline Adrenaline rush & 1 & 1 & $\mathrm{RH}$ & 01/05/2017 02:10 PM & $\mathrm{RH}$ & 01/05/2017 02:13 PM \\
\hline Self-harm \& self-esteem & 0 & 0 & $\mathrm{RH}$ & 25/04/2017 10:46 AM & $\mathrm{RH}$ & 25/04/2017 10:46 AM \\
\hline Self-punishment & 1 & 2 & $\mathrm{RH}$ & 25/04/2017 10:47 AM & $\mathrm{RH}$ & 27/01/2017 10:36 AM \\
\hline Feeling rejected & 2 & 2 & $\mathrm{RH}$ & 21/04/2017 10:33 AM & $\mathrm{RH}$ & 25/04/2017 10:46 AM \\
\hline Worthlessness & 2 & 2 & $\mathrm{RH}$ & 25/04/2017 10:47 AM & $\mathrm{RH}$ & 25/04/2017 10:49 AM \\
\hline Self-harm \& self-blame & 0 & 0 & $\mathrm{RH}$ & 25/04/2017 10:57 AM & $\mathrm{RH}$ & 25/04/2017 10:57 AM \\
\hline Self blame & 3 & 3 & RH & 25/04/2017 10:47 AM & $\mathrm{RH}$ & 25/04/2017 10:59 AM \\
\hline Gain control & 2 & 2 & $\mathrm{RH}$ & 21/04/2017 10:33 AM & $\mathrm{RH}$ & 27/01/2017 10:47 AM \\
\hline Persecuted & 4 & 4 & $\mathrm{RH}$ & 21/04/2017 10:33 AM & $\mathrm{RH}$ & 25/04/2017 10:55 AM \\
\hline$\forall$ Self-harm as a release & 11 & 18 & $\mathrm{RH}$ & 21/04/2017 10:33 AM & RH & 13/05/2017 10:10 AM \\
\hline Calming & 1 & 2 & $\mathrm{RH}$ & 13/05/2017 10:09 AM & $\mathrm{RH}$ & 13/05/2017 10:09 AM \\
\hline Anger & 4 & 5 & $\mathrm{RH}$ & 21/04/2017 10:33 AM & $\mathrm{RH}$ & 14/02/2017 11:05 AM \\
\hline
\end{tabular}


Painful memories $\mathbf{x}$

$\leq$ Internals $\backslash \backslash$ Transcripts \\P00008 $>$ - 2 references coded [1.76\% Coverage]

Reference $1-0.95 \%$ Coverage

My mind was going into overload and I was thinking of all the crap things that had happened to me in my life and how crap my future was going to be so I thought 'sod it I don't want to be here anymore'

\title{
Reference $2-0.81 \%$ Coverage
}

A: He's the person (step-father) who's always angered me the most and upset me the most and made me feel down like I don't want to be alive and that's what shows up first

$\leq \mid$ Internals $\backslash \backslash$ Transcripts $\backslash \backslash P 00017>-\$ 1$ reference coded $[0.40 \%$ Coverage]

Reference $1-0.40 \%$ Coverage

I was sat thinking about times when things have gone wrong before

\author{
Self blame $\mathbf{x}$

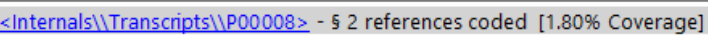 \\ Reference $1-1.44 \%$ Coverage \\ T: So are you putting the depressed feelings as happening first before the suicidal feelings? \\ A: Well yeah because I wouldn't be feeling suicidal if I wasn't depressed \\ T: And what's that like being depressed all the time? \\ A: Well it's like I feel like a complete failure and I can't do anything right \\ Reference $2-0.36 \%$ Coverage \\ I feel as though I've done something wrong and have to punish myself for it \\ $\leq \mid$ Internals $\backslash \backslash T$ ranscripts $\backslash P 00017>-\$ 4$ references coded [3.29\% Coverage] \\ Reference $1-1.19 \%$ Coverage \\ I was like oh my days I've just ruined there day and I started thinking about other times I've done that \\ and I was thinking I've done it again and ruined their whole day again and it led to this \\ Reference $2-0.56 \%$ Coverage \\ I was thinking it seems to be me that's the cause of anything that went wrong in the family
}

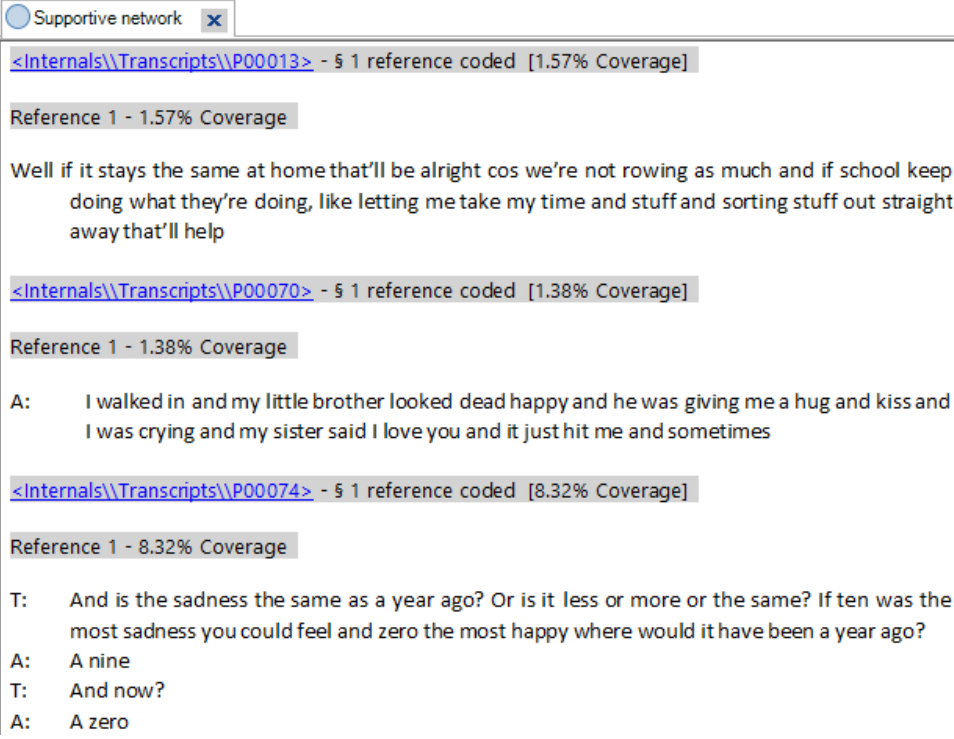

Well if it stays the same at home that'll be alright cos we're not rowing as much and if school keep doing what they're doing, like letting me take my time and stuff and sorting stuff out straight away that'll help

$\leq \mid$ Internals $\backslash \backslash$ Transcripts $\backslash \backslash P 00070>-\$ 1$ reference coded [1.38\% Coverage]

Reference $1-1.38 \%$ Coverage

A: I walked in and my little brother looked dead happy and he was giving me a hug and kiss and I was crying and my sister said I love you and it just hit me and sometimes

$\leq \mid$ Internals $\backslash \backslash T$ ranscripts $\backslash \backslash P 00074>-\$ 1$ reference coded [8.32\% Coverage]

Reference $1-8.32 \%$ Coverage

$\mathrm{T}$ : And is the sadness the same as a year ago? Or is it less or more or the same? If ten was the most sadness you could feel and zero the most happy where would it have been a year ago?

A: A nine

T: And now?

A: A zero 